IJMMS 32:11 (2002) 641-699

PII. S0161171202207188

http://ijmms.hindawi.com

(c) Hindawi Publishing Corp.

\title{
THE DOUBLE BUBBLE PROBLEM IN SPHERICAL SPACE AND HYPERBOLIC SPACE
}

\author{
ANDREW COTTON and DAVID FREEMAN
}

Received 28 July 2002

We prove that the standard double bubble is the least-area way to enclose and separate two regions of equal volume in $\mathbb{t}^{3}$, and in $S^{3}$ when the exterior is at least ten percent of $S^{3}$.

2000 Mathematics Subject Classification: 49Q10, 53A10.

1. Introduction. The double bubble conjecture states that the least-area way to enclose and separate two given volumes is a "standard double bubble" consisting of three spherical caps meeting at 120-degree angles (see Figure 1.1). The conjecture was proven for $\mathbb{R}^{2}$ by the 1990 Williams College NSF "SMALL" undergraduate research Geometry Group [6]. The equal-volumes case for $\mathbb{R}^{3}$ was proven in 1995 by Hass et al. $[8,9]$. In 2000, Hutchings et al. [11] announced a proof of the general case in $\mathbb{R}^{3}$. The 1999 "SMALL" Geometry Group [17] generalized this result to $\mathbb{R}^{4}$ and, for the case where the larger volume is more than twice the smaller, to $\mathbb{R}^{n}$. In $\mathbb{R}^{5}$ and higher dimensions, even the case of equal volumes remains open. The 2000 edition of Morgan's book [13] provides a good general reference on the subject, including all of these results.

In 1995, Masters [12] proved the conjecture on the two-sphere $S^{2}$. In Theorem 2.7, we note that the latest proof for $\mathbb{R}^{2}$ applies to the hyperbolic plane $\mathbb{t}^{2}$ and immiscible fluids as well.

In this paper, we prove certain cases of the double bubble conjecture in the threesphere $S^{3}$ and three-dimensional hyperbolic space $\mathbb{M}^{3}$.

THEOREM 1.1. A least-area enclosure of two equal volumes in $S^{3}$ which add up to at most 90 percent of the total volume of $S^{3}$ must be the (unique) standard double bubble.

THEOREM 1.2. A least-area enclosure of two equal volumes in $\mathbb{M}^{3}$ must be the (unique) standard double bubble.

The proof follows the same outline as the proof for $\mathbb{R}^{3}$ by Hutchings et al. [11], including component bounds, structure theory, and an instability argument.

A major difficulty in such proofs is that one cannot assume a priori that either of the enclosed regions or the exterior is connected. If one tries to require each region to be connected, it might disconnect in the minimizing limit, as thin connecting tubes shrink away. In principle, the Hutchings component bounds [10, Sections 3 and 4] extend to the $n$-sphere $S^{n}$ and $n$-dimensional hyperbolic space $\mathbb{\boxplus}^{n}$, but the formulae are difficult to work with. We consider only the cases in $S^{3}$ and $\mathbb{u}^{3}$ in which the two 


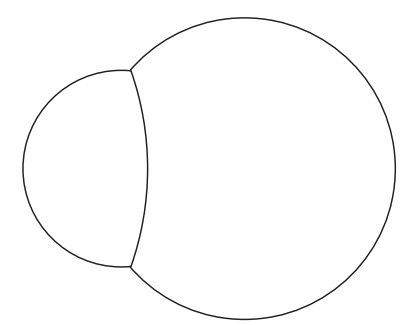

FIGURE 1.1. The standard double bubble, consisting of three spherical caps meeting at 120-degree angles, is the conjectured least-area surface that encloses two given volumes in $\mathbb{R}^{n}, S^{n}$, and $\mathbb{\boxplus}^{n}$.

regions to be enclosed have the same volume $v$. In Section 4 , we reduce the condition implying both regions connected to an inequality, $F(v)>0$ (Proposition 4.8). In Section 5, we prove that the function $F(v)$ is positive for small volumes by making Euclidean approximations to $S^{3}$ and $\mathbb{H}^{3}$ (Propositions 5.5 and 5.11). For large volumes in $S^{3}$, the region exterior to the two volumes becomes very small and may become disconnected for all we know. For large volumes in $\mathbb{t}^{3}$, we use asymptotic analysis to show that $F(v)$ remains positive (Proposition 5.19). We prove that $F(v)$ is positive for intermediate volumes in both cases by bounding the derivative $F^{\prime}(v)$ and checking a finite number of points by computer (Propositions 5.8 and 5.14). We conclude that all regions of the equal-volume double bubble are connected in $S^{3}$ when the exterior is at least 10 percent of $S^{3}$, and in $\mathbb{\boxplus}^{3}$ for all volumes (Propositions 5.1 and 5.2).

In Section 6, we consider the structure of area-minimizing double bubbles in $S^{n}$ and $\mathbb{M}^{n}$. We adapt an argument of Foisy [5, Theorem 3.6] to show that an area-minimizing bubble in $\mathbb{q}^{n}$ must intersect its axis of symmetry (Proposition 6.8). As a result, the Hutchings structure theorem [10, Section 5] carries over exactly to $\mathbb{\boxplus}^{n}$ (Theorem 6.10 ). In $S^{n}$, we have no corresponding method of ruling out bubbles which do not intersect the axis, and any or all of the three regions may be disconnected. In our structure theorem for $S^{n}$ (Theorem 6.5), we consider only cases when we know that one region is connected, and classify bubbles based on whether this region intersects part, all, or none of the axis of symmetry.

Finally, we use the instability argument of Hutchings et al. [11, Proposition 5.2] to show, in Section 7, that a nonstandard competitor in which all regions are connected is unstable and thus cannot be a minimizer (Propositions 7.3 and 7.7). This argument supposes that there is a nonstandard minimizer, and produces infinitesimal isometric motions on pieces of the bubble which maintain volume and reduce area. For connected regions we generalize this method directly to $S^{n}$ (Theorem 7.2) and, with some more work, to $\mathbb{a}^{n}$ (Theorem 7.6), where we need to use all three types of isometries (elliptical, parabolic, and hyperbolic). This proves the double bubble theorem for the cases in which we know all regions to be connected.

\subsection{Open questions}

QUESTION 1. Are all but the smallest region of a minimizing double bubble in $\mathbb{Q}^{3}$ or $S^{3}$ always connected? 
The Hutchings theory (see [11, Proposition 6.2] and [17, Proposition 2.5]) implies that in $\mathbb{R}^{3}$ and $\mathbb{R}^{4}$, the larger of the two enclosed regions is always connected. By scaling, in $\mathbb{R}^{n}$ one needs to consider only the one-parameter family of double bubbles of unit total volume. In $S^{n}$ and $\mathbb{q}^{n}$, the unequal-volumes case is a two-parameter family. Our generalization of the Hutchings theory reduces the condition that the larger region of a double bubble enclosing volumes $v$ and $w$ is connected to an inequality, $F(v, w)>0$ for $v \geq w$. This function $F$ will be even more difficult to work with than the single-variable function obtained for the equal-volume case, but our methods of Section 5 may generalize; for instance, it would be relatively easy to do a computer plot. (This has recently been done by the 2001 "SMALL" Geometry Group [4].) We do note that since the larger region is always connected in $\mathbb{R}^{3}$ and $\mathbb{R}^{4}$, it must be connected in $S^{3}, S^{4}, \mathbb{t}^{3}$, and $\mathbb{q}^{4}$ for two small volumes. Calculating a precise value for "small," however, may be difficult.

QUESTION 2. Are all competing double bubbles in $S^{n}$ and $\mathbb{\boxplus}^{n}$ unstable if at most one region is disconnected?

Hutchings et al. [11, Section 5] show that a competitor in $\mathbb{R}^{n}$ in which the disconnected region has at most two components is unstable. Reichardt et al. [17, Section 8] generalize their method to show that the bubble is unstable if the disconnected region has any number of components. The proofs in both cases rely on certain properties of constant-mean-curvature (Delaunay) hypersurfaces in $\mathbb{R}^{n}$. A generalization for $S^{n}$ and $\mathbb{\square}^{n}$ most likely would use properties of Delaunay surfaces in those spaces. (Treatments of these surfaces can be found in $[3,7,18]$.) The $\mathbb{R}^{n}$ proofs also make extensive use of planar Euclidean geometry, and many steps may not generalize to non-Euclidean spaces.

To prove the double bubble conjecture in the general case for $S^{3}$ and $\mathbb{a}^{3}$, it would suffice to show that the answer to both of the above questions is Yes. To prove the double bubble conjecture in $S^{n}$ and $\mathbb{a}^{n}$ for the case in which the smallest region is less than half as large as the others, it would suffice to show that the answer to Question 2 is Yes, for in these cases all but the smallest region must be connected (see [10, Theorem 3.5, Corollary 3.10]).

Finally, we make the following conjecture for small volumes in any smooth Riemannian manifold $M$ with compact quotient $M / \Gamma$ by the isometry group $\Gamma$.

CONJECTURE 1.3. On any smooth $n$-dimensional Riemannian manifold $M$ with compact quotient $M / \Gamma$ by the isometry group $\Gamma$, the least-area enclosure of two small volumes is a standard double bubble.

For $n=2$, a nontrivial small stable double bubble is known to be standard [15]. For $n=3$ and $n=4$, for fixed volume ratio a small double bubble in a smooth, closed, flat Riemannian manifold is known to be standard [2].

2. Existence and regularity. The existence of area-minimizing double bubbles (Proposition 2.3) is a fairly standard result of geometric measure theory. The fact that a minimizing double bubble is a surface of revolution about a line (Proposition 2.4) has long been known and was proven by Foisy [5] and Hutchings [10, Theorem 2.6, 
Lemma 2.9]. The proof of uniqueness of the standard double bubble in $S^{n}$ and $\mathbb{a}^{n}$ (Proposition 2.6) is adapted from Masters' proof for $S^{2}$ [12, Theorem 2.2]. Finally, Theorem 2.7 notes that the latest proof (after Hutchings) for the double bubble conjecture in $\mathbb{R}^{2}$ (see [14]) carries over to $\mathbb{H}^{2}$.

We begin, however, with a precise definition of "double bubble."

DEFINITION 2.1. Let $M$ be a smooth $n$-dimensional Riemannian manifold. A double bubble in $M$ is the union of the topological boundaries of two disjoint regions of prescribed volumes. A smooth double bubble $\Sigma$ in $M$ is a piecewise smooth oriented hypersurface consisting of three compact pieces $\Sigma_{1}, \Sigma_{2}$, and $\Sigma_{3}$ (smooth up to boundary), with a common $(n-2)$-dimensional smooth boundary $C$ such that $\Sigma_{1}+\Sigma_{2}$ (resp., $\Sigma_{3}+\Sigma_{2}$ ) encloses a region $R_{1}$ (resp., $R_{2}$ ) of prescribed volume $v_{1}$ (resp., $v_{2}$ ). None of these is assumed to be connected.

DEFINITION 2.2. A standard double bubble in $\mathbb{R}^{n}, S^{n}$, or $\mathbb{a}^{n}$ is a smooth double bubble in which $\Sigma_{1}, \Sigma_{2}$, and $\Sigma_{3}$ are spherical surfaces meeting in an equiangular way along a given $(n-2)$-dimensional sphere $C$.

Proposition 2.3 [13, Theorem 13.4, Remark before Proposition 13.8]. In a smooth Riemannian manifold $M$ with compact quotient $M / \Gamma$ by the isometry group $\Gamma$, for any two volumes $v$ and $w$ (whose sum is less than or equal to $\operatorname{vol}(M)$ if $M$ is compact), there exists a least-area enclosure of the two volumes. This enclosure consists of smooth constant-mean-curvature hypersurfaces, except possibly for a set of measure zero.

Proposition 2.4 [10, Lemma 2.9, Remark 3.8 and following]. For $n \geq 3$, an areaminimizing double bubble in $S^{n}$ or $\mathbb{Q}^{n}$ is a hypersurface of revolution about a line.

Proof. The proof is the same (adapted to $S^{n}$ and $\mathbb{q}^{n}$ instead of $\mathbb{R}^{n}$ ) as those of Foisy [5] and Hutchings [10, Theorem 2.6, Lemma 2.9].

The standard double bubble is said to consist of three spherical caps; however, these caps need not be pieces of actual spheres. We thus define precisely what we mean when we say a surface is "spherical."

DEFINITION 2.5. The term spherical denotes a surface for which all principal curvatures are equal. The term circular denotes a constant-curvature curve.

The only $(n-1)$-dimensional spherical surfaces in $S^{n}$ are spheres. Such surfaces in $\mathbb{R}^{n}$ are spheres and planes, while those in $\mathbb{\boxplus}^{n}$ are spheres, horospheres, hypospheres, and geodesic planes.

Proposition 2.6. For two prescribed volumes $v, w$ (with $v+w<\operatorname{vol}\left(S^{n}\right)$ ), there is a unique standard double bubble in $S^{n}$ (up to isometries) consisting of three spherical caps meeting at 120 degrees that encloses volumes $v$ and $w$.

For two prescribed volumes $v, w$, there is a unique standard double bubble in $\mathbb{M}^{n}$ (up to isometries) consisting of three spherical caps meeting at 120 degrees that encloses volumes $v$ and $w$. The outer two caps are pieces of spheres, and the middle cap may be any spherical surface. 


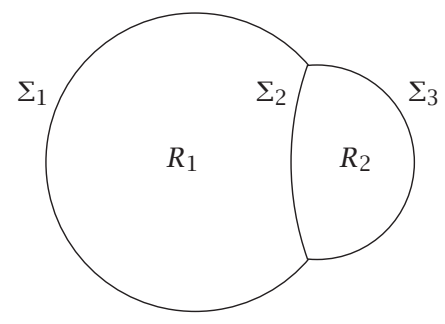

FiguRE 2.1. Construction of a standard double bubble from three spherical caps.

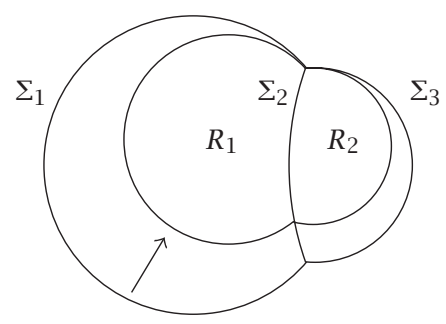

FIGURE 2.2. Increasing the curvature of $\Sigma_{1}$ while keeping the curvature of $\Sigma_{2}$ fixed increases the curvature of $\Sigma_{3}$. The volumes of $R_{1}$ and $R_{2}$ both decrease.

Proof. Masters [12, Theorem 2.2] proved the existence and uniqueness of the standard double bubble in $S^{2}$; this result generalizes directly to $S^{n}$ merely by considering spherical caps instead of circles. We use similar methods for $\mathbb{t}^{n}$. The main idea of the proof is to parameterize double bubbles by the mean curvatures of one of the outer caps and the middle cap.

Consider two mean curvatures (sums of principal curvatures) $H_{1} \in(n-1, \infty)$ and $H_{2} \in[0, \infty)$. Draw two spherical caps $\Sigma_{1}, \Sigma_{2}$ with these mean curvatures, meeting at 120 degrees, so that $\Sigma_{1}$ has positive mean curvature when considered from the side on which the angle is measured and $\Sigma_{2}$ has negative mean curvature when considered from this side. It is obvious that the caps must meet up, since $\Sigma_{1}$ is a portion of a sphere (because $H_{1}>n-1$ ). Denote the enclosed region $R_{1}$. Complete this figure to a double bubble with a third spherical cap $\Sigma_{3}$ that meets the other two at 120 degrees at their boundary, enclosing a second region $R_{2}$. Note that $\Sigma_{2}$ will necessarily be the middle cap. (See Figure 2.1.) Obviously there is at most one way to do this. To see that this can always be done, note that if $H_{2}$ is equal to zero, then $\Sigma_{1}$ and $\Sigma_{3}$ are identical. As we increase $H_{2}$ with $H_{1}$ fixed, the mean curvature of $\Sigma_{3}$ increases, as shown in Figure 2.3. Thus $\Sigma_{3}$ has mean curvature greater than or equal to $H_{1}$ and is thus a portion of a sphere, so there is no problem with surfaces going off to infinity without meeting up.

Let $V_{1}$ be the volume of $R_{1}$ and $V_{2}$ be the volume of $R_{2}$. Define a map $F:(n-1, \infty) \times$ $[0, \infty) \rightarrow\left\{(x, y) \in \mathbb{R}_{>0} \times \mathbb{R}_{>0} \mid x \geq y\right\}$ such that $F\left(H_{1}, H_{2}\right)=\left(V_{1}, V_{2}\right)$. As can be seen in Figures 2.2 and 2.3, with $H_{2}$ fixed, as $H_{1}$ increases, both $V_{1}$ and $V_{2}$ decrease. With $H_{1}$ 


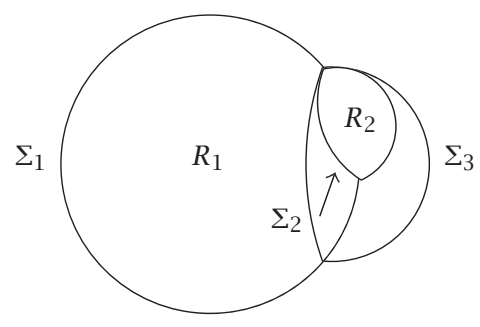

FIGURE 2.3. Increasing the curvature of $\Sigma_{2}$ while keeping the curvature of $\Sigma_{1}$ fixed increases the curvature of $\Sigma_{3}$. The volume of $R_{1}$ increases and the volume of $R_{2}$ decreases.

fixed, as $H_{2}$ increases, $V_{1}$ increases and $V_{2}$ decreases. (Note that $V_{2} \leq V_{1}$, with equality only at $H_{2}=0$.) Thus we conclude that the map $F$ is injective.

To show that $F$ is surjective, we first note that the map is continuous. We now consider limiting cases. With $H_{1}$ fixed, as $H_{2}$ goes to zero (and $\Sigma_{2}$ becomes a geodesic plane), the two volumes enclosed become equal. With $H_{2}$ fixed, as $H_{1}$ approaches infinity, both volumes $V_{1}$ and $V_{2}$ approach zero. With $H_{1}$ fixed, as $H_{2}$ goes to infinity, $V_{1}$ approaches the volume of a sphere of mean curvature $H_{1}$ and $V_{2}$ goes to zero. With $H_{2}$ fixed, as $H_{1}$ decreases, $V_{1}$ increases without bound. By continuity of $F$, all volumes $\left(V_{1}, V_{2}\right)$ are achieved by our construction. Note that since $V_{1} \geq V_{2}, V_{1}$ must become infinite first, which will happen when $H_{1}=n-1$ and $\Sigma_{1}$ becomes a horosphere. Thus $F$ is surjective. In addition, each outer cap must be a sphere and not a horosphere or a hyposphere.

By construction, the total volume of the double bubble is greater than the total volume of a spherical surface with mean curvature $H_{1}$, so if $H_{1} \leq n-1$, the enclosed volume is infinite. Thus we achieve each pair of volumes $V_{1}, V_{2}$ with a standard double bubble and that every finite-volume standard double bubble is achieved in our construction, so we have the stated result.

\subsection{Proof of the double bubble conjecture for $\mathbb{\boxplus}^{2}$}

THEOREM 2.7. The least-area way to enclose two volumes $v$ and $w$ in $\mathbb{Q}^{2}$ is with a standard double bubble, unique up to isometries of $\mathbb{\boxplus}^{2}$.

Proof. The proof that the minimizer is the standard double bubble is identical to that given for $\mathbb{R}^{2}$ by Hutchings [14]. The uniqueness of the standard double bubble for two given volumes follows from Proposition 2.6.

The proof that works for $\mathbb{R}^{2}$ and $\mathbb{\boxplus}^{2}$ fails for $S^{2}$ because the least-area function for double bubbles is not increasing with volume enclosed; for certain volumes, it is possible to enclose more volume with less area. This proof also applies to the immiscible fluids problem in $\mathbb{R}^{2}$ and $\mathbb{t}^{2}$ (see [13, Chapter 16]), answering a problem posed by Greenleaf, Barber, Tice, and Wecht [19, problem 6].

3. Volumes and areas in $S^{n}$ and $\mathbb{\natural}^{n}$. In order to calculate component bounds in Sections 4 and 5, we will need to know the area and volume of spheres and double 
bubbles in $S^{n}$ and $\mathbb{M}^{n}$. We begin with the formulae for spheres, which we then use to calculate area and volume for the standard double bubble enclosing two equal volumes in $S^{3}$ and $\mathbb{\boxplus}^{3}$.

The surface area of an $(n-1)$-dimensional sphere of radius $r$ in $n$-dimensional Euclidean space $\mathbb{R}^{n}$ is $A_{\mathbb{R}^{n}}(r)=n \alpha_{n} r^{n-1}$, where $\alpha_{n}$ is the volume of a ball of unit radius

$$
\alpha_{n}=\frac{\pi^{n / 2}}{(n / 2) !} .
$$

In Euclidean space, differential length in a direction tangent to a sphere is $r d \theta$, while in spherical space and hyperbolic space, this differential length is $\sin r d \theta$ and $\sinh r d \theta$, respectively. We thus have the following formulae.

REMARK 3.1. The surface area of $(n-1)$-spheres of radius $r$ in $S^{n}$ and $\mathbb{M}^{n}$ are

$$
A_{S^{n}}(r)=n \alpha_{n} \sin ^{n-1} r, \quad A_{\mathbb{\natural} n}(r)=n \alpha_{n} \sinh ^{n-1} r .
$$

REMARK 3.2. The volumes of $n$-balls of radius $r$ in $S^{n}$ and $\mathbb{Q}^{n}$ are, for $n=2$ and $n=3$,

$$
\begin{aligned}
V_{S^{2}}(r) & =2 \pi(1-\cos r), \\
V_{\mathbb{H}^{2}}(r) & =2 \pi(\cosh r-1), \\
V_{S^{3}}(r) & =\pi(2 r-\sin 2 r), \\
V_{\mathbb{H}^{3}}(r) & =\pi(\sinh 2 r-2 r) .
\end{aligned}
$$

These volume formulae are obtained by integrating the area formulae in Remark 3.1.

REMARK 3.3. For an $(n-1)$-sphere of radius $r$ in $S^{n}$, the mean curvature $d A / d V$ is equal to $(n-1) \cot r$. In $\mathbb{Q}^{n}$, the mean curvature is equal to $(n-1) \operatorname{coth} r$, and in $\mathbb{R}^{n}$ it is equal to $(n-1) / r$.

Proof. The volume of a sphere of radius $r$ in $S^{n}$ is $\int_{0}^{r} A\left(r^{\prime}\right) d r^{\prime}$. The derivative $d A / d V$ is $(d A / d r) /(d V / d r)=A^{\prime}(r) / A(r)$. From Remark 3.1, this is equal to $(n-1) \sin ^{n-1} r \cos r / \sin ^{n} r$, or $(n-1) \cot r$. The same calculation in $\mathbb{Q}^{n}$ gives $d A / d V=$ $(n-1) \operatorname{coth} r$ and in $\mathbb{R}^{n}$ gives $(n-1) / r$.

For the following derivations, we will refer to Figure 3.1, which shows the generating curve for a double bubble enclosing two equal volumes. When revolved about the axis of revolution, both arcs become spherical caps, line $\overline{A B}$ becomes a flat disc, and triangle $A B C$ becomes a cone. We calculate the surface area of the bubble by adding up the areas of the two caps and the disc, and we calculate the volume of one half adding the volume of the cone to that of the fraction of the sphere subtended by one cap. Throughout, we use standard formulae from spherical and hyperbolic trigonometry, which can be found in Ratcliffe's book [16] or in any introductory text on non-Euclidean geometry.

Proposition 3.4. Construct a standard double bubble enclosing two regions of equal volume by gluing together two identical spherical caps of radius $r$ and a flat disc such that the three pieces meet at 120-degree angles (see Figure 3.1). The surface 


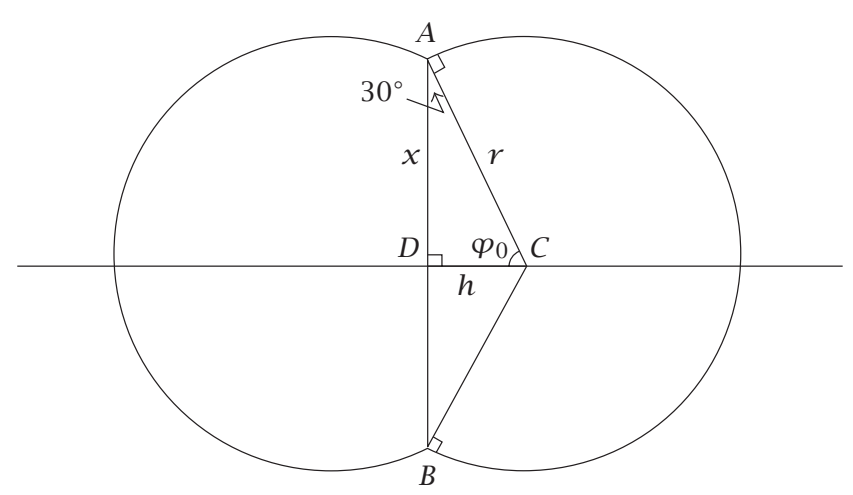

FIGURE 3.1. Generating curve for a standard double bubble enclosing two equal volumes. $\varphi_{0}$ is $60^{\circ}$ in $\mathbb{R}^{n}$, greater than $60^{\circ}$ in $S^{n}$, and less than $60^{\circ}$ in $\mathbb{a}^{n}$.

area of this bubble in $S^{3}$ is

$$
A_{S}(r, r)=4 \pi \sin ^{2} r\left(1 \pm \frac{\sqrt{2} \cos r}{\sqrt{7+\cos 2 r}}\right)+2 \pi\left(1 \mp \frac{2 \sqrt{2} \cos r}{\sqrt{7+\cos 2 r}}\right)
$$

where the top sign of \pm and $\mp$ is used for $r \leq \pi / 2$ and the bottom sign is used for $r>\pi / 2$.

The surface area of the bubble in $\mathbb{\boxplus}^{3}$ is

$$
A_{h}(r, r)=4 \pi \sinh ^{2} r\left(1+\frac{\sqrt{2} \cosh r}{\sqrt{7+\cosh 2 r}}\right)+2 \pi\left(\frac{2 \sqrt{2} \cosh r}{\sqrt{7+\cosh 2 r}}-1\right)
$$

Proof. We derive the formula for $S^{3}$. The derivation for $\mathbb{M}^{3}$ is entirely analogous, and in fact somewhat simpler, since hyperbolic trigonometric functions are not periodic.

From Figure 3.1 and spherical trigonometry, we have

$$
\tan x=\tan r \cos 30^{\circ}, \quad \sin \varphi_{0}=\frac{\sin x}{\sin r},
$$

which we can solve for $x$ and $\varphi_{0}$. Note that if $r=\pi / 2$, then $\overline{A C}$ and $\overline{B C}$ are part of the same great circle, so $\varphi_{0}=\pi / 2$ and $x=\pi / 2$. If $r>\pi / 2$, then $\varphi_{0}>\pi / 2$.

We set up our integrals using spherical coordinates in $S^{3}$. In this coordinate system, the integral for the area of one of the identical spherical caps is

$$
\int_{0}^{2 \pi} \int_{0}^{\pi-\varphi_{0}} \sin ^{2} r \sin \varphi d \theta d \varphi
$$


which evaluates to

$$
2 \pi \sin ^{2} r\left(1 \pm \frac{\sqrt{2} \cos r}{\sqrt{7+\cos 2 r}}\right)
$$

where the \pm is determined by $\varphi_{0}$ (positive for $\varphi_{0}<\pi / 2$ and negative for $\varphi_{0}>\pi / 2$ ).

The area of the disc separating the two bubbles is just that of a circle of radius $x$, $2 \pi(1-\cos x)$, which evaluates to

$$
2 \pi\left(1 \mp \frac{2 \sqrt{2} \cos r}{\sqrt{7+\cos 2 r}}\right)
$$

where the $\mp$ is determined by $\varphi_{0}$ (negative for $\varphi_{0}<\pi / 2$ and positive for $\varphi_{0}>\pi / 2$ ). Adding the two spherical caps to one flat disc gives us the surface area of the bubble.

Proposition 3.5. Construct a standard double bubble enclosing two regions of equal volume by gluing together two identical spherical caps of radius $r$ and a flat disc such that the three pieces meet at 120-degree angles (see Figure 3.1). In $S^{3}$, the volume $V_{s}(r, r)$ of one of the enclosed regions is

$$
\begin{aligned}
V_{s}(r, r)= & \frac{\pi}{2}(2 r-\sin 2 r)\left(1+\frac{\sqrt{2} \cos r}{\sqrt{7+\cos 2 r}}\right) \\
& +\pi\left(\tan ^{-1}\left(\frac{\sqrt{2} \sin r}{\sqrt{7+\cos 2 r}}\right)-\frac{\sqrt{2} r \cos r}{\sqrt{7+\cos 2 r}}\right)
\end{aligned}
$$

and in $\mathbb{\boxplus}^{3}$, the volume $V_{h}(r, r)$ of one of the enclosed regions is

$$
\begin{aligned}
V_{h}(r, r)= & \frac{\pi}{2}(\sinh 2 r-2 r)\left(1+\frac{\sqrt{2} \cosh r}{\sqrt{7+\cosh 2 r}}\right) \\
& +\pi\left(\frac{\sqrt{2} r \cosh r}{\sqrt{7+\cosh 2 r}}-\tanh ^{-1}\left(\frac{\sqrt{2} \sinh r}{\sqrt{7+\cosh 2 r}}\right)\right) .
\end{aligned}
$$

Proof. Again, we derive the formula for $S^{3}$ only, as the derivation for $\mathbb{\natural}^{3}$ is analogous. To simplify our calculations, we compute $V_{s}(r, r)$ using integrals for $r \leq \pi / 2$, while for larger $r$ we find $V_{s}(r, r)$ in terms of $V_{s}(\pi-r, \pi-r)$.

We split each region of the double bubble into a portion of a sphere whose cross section is bounded by arc $A B$ and geodesic segments $\overline{A C}$ and $\overline{B C}$, and a cone whose cross-section is triangle $A B C$. Again, we set up our integrals in spherical coordinates in $S^{3}$. The integral to find the volume of the spherical part is straightforward; it is

$$
\int_{0}^{2 \pi} \int_{0}^{\pi-\varphi_{0}} \int_{0}^{r} \sin ^{2} \rho \sin \varphi d \rho d \varphi d \theta
$$


which evaluates to

$$
\frac{\pi}{2}(2 r-\sin 2 r)\left(1+\frac{\sqrt{2} \cos r}{\sqrt{7+\cos 2 r}}\right)
$$

To set up the integral for the cone, we must consider the range of $\rho$ as $\varphi$ ranges from 0 to $\varphi_{0}$. Consider a geodesic segment from $C$ meeting $\overline{A D}$ at $E$ such that $\angle E C D=\varphi$. We then have $\tan C E=\tan C D \sec \varphi$, and from our original triangle $A B C$, we have $\sin C D=\sin r \sin 30^{\circ}$. Therefore $\rho$ ranges from 0 to the length of $\overline{C E}$, which is $\tan ^{-1}\left(\sec \varphi\left(\sin r / \sqrt{4-\sin ^{2} r}\right)\right)$. Thus the integral for the volume of the cone is

$$
\int_{0}^{2 \pi} \int_{0}^{\varphi_{0}} \int_{0}^{\tan ^{-1}\left(\sec \varphi\left(\sin r / \sqrt{4-\sin ^{2} r}\right)\right.} \sin ^{2} \rho \sin \varphi d \rho d \varphi d \theta
$$

which evaluates to

$$
\pi\left(\tan ^{-1}\left(\frac{\sqrt{2} \sin r}{\sqrt{7+\cos 2 r}}\right)-\frac{\sqrt{2} r \cos r}{\sqrt{7+\cos 2 r}}\right) .
$$

Adding the volume of the cone to that of the sphere gives the volume of one half of the double bubble.

For $r>\pi / 2$, we find the volume in terms of the formula for $r \leq \pi / 2$. The completion of the disc separating the two bubbles divides $S^{3}$ into two hemispheres. On each hemisphere, the region exterior to the double bubble is bounded by a portion of a sphere of radius $\pi-r$. By supplementary angles, this portion of the sphere meets the disc at a 60-degree angle. Now consider the completion of the sphere of radius $\pi-r$. The portion added is a spherical cap cut off by a flat disc at a 120-degree angle, so its volume is $V_{s}(\pi-r, \pi-r)$. The volume of the exterior is thus the volume of the sphere of radius $\pi-r$ minus the portion added: $\pi(2(\pi-r)-\sin 2(\pi-r))-V_{s}(\pi-r, \pi-r)=$ $2 \pi^{2}-\pi(2 r-\sin 2 r)-V_{s}(\pi-r, \pi-r)$. The volume of each region of the original double bubble is thus the volume of the entire hemisphere $\left(\pi^{2}\right)$ minus the volume of the exterior on that hemisphere: $V_{s}(\pi-r, \pi-r)+\pi(2 r-\sin 2 r)-\pi^{2}$. Simple algebraic manipulation shows that this expression is equivalent to the formula in the theorem statement.

We will also need formulae for the area and volume of a standard double bubble in $\mathbb{R}^{3}$ enclosing two equal volumes. This is easy to calculate from the drawing in Figure 3.1, so we omit the algebra here.

REMARK 3.6. Construct a standard double bubble enclosing two regions of equal volume by gluing together two identical spherical caps of radius $r$ and a flat disc such that the three pieces meet at 120-degree angles. The surface area of this bubble in $\mathbb{R}^{3}$ is

$$
A_{e}(r, r)=\frac{27}{4} \pi r^{2}
$$

and the volume of one of the enclosed regions is

$$
V_{e}(r, r)=\frac{9}{8} \pi r^{3}
$$


Note that these formulae give

$$
A_{e}(v, v)=(36 \pi)^{1 / 3} v^{2 / 3} .
$$

Finally, we derive formulae for the curvature of double bubbles in all three spaces. The method is the same as in Remark 3.3, and we omit the algebra.

REMARK 3.7. For a standard double bubble in $S^{3}$, the mean curvature $d A / d V$ is equal to $4 \cot r$. In $\mathbb{t}^{3}$, the mean curvature is equal to $4 \operatorname{coth} r$, and in $\mathbb{R}^{3}$ it is equal to $4 / r$.

4. Component bounds for area-minimizing double bubbles. In this section, we develop in $S^{n}$ and $\mathbb{\boxplus}^{n}$ the Hutchings theory of bounds [10] on the number of components of area-minimizing double bubbles. Proposition 4.8 gives a new, convenient statement of the basic estimate.

\subsection{Concavity of the least-area function and applications}

Proposition 4.1 [10, Theorem 3.9]. For $n \geq 3$, the least area required to partition the sphere $S^{n}$ into three volumes is strictly concave on every line in the simplex

$$
\left\{v_{1}+v_{2}+v_{3}=\operatorname{vol}\left(S^{n}\right)\right\} .
$$

COROLlary 4.2 [10, Corollary 3.10]. Consider an area-minimizing partition of $S^{n}$ into volumes $v_{1}, v_{2}, v_{3}$ (with $v_{1}+v_{2}+v_{3}=\operatorname{vol}\left(S^{n}\right)$ ). If $v_{i}>2 v_{j}$ for some $i, j$, then the region enclosing volume $v_{i}$ is connected.

Hutchings [10, Section 3] proves the following results for $\mathbb{R}^{n}$. He notes [10, Remark $3.8]$ that the proofs carry over to $\mathbb{U}^{n}$ with minor rewording, and that one needs to check only that the least area function for one volume is concave. This concavity is obvious from Remark 3.3, since coth $r$ is decreasing in $r$.

Proposition 4.3 [10, Theorem 3.2]. For $n \geq 3$, the least area $A(v, w)$ of a double bubble enclosing volumes $v, w$ in $\mathbb{\boxplus}^{n}$ is a strictly concave function.

COROLlary 4.4 [10, Corollary 3.3]. For $n \geq 3$, the least area function $A(v, w)$ of double bubbles enclosing volumes $v, w$ in $\mathbb{t}^{n}$ is strictly increasing in $v$ and $w$.

COROllary 4.5 [10, Theorem 3.4]. An area-minimizing double bubble in $\mathbb{\boxplus}^{n}$ has a connected exterior.

Proposition 4.6 [10, Theorem 3.5]. If $v>2 w$, then in any least-area enclosure of volumes $v$ and $w$ in $\mathbb{\boxplus}^{n}$, the region of volume $v$ is connected.

4.2. Component bound formulae. Let $A(x)$ denote the minimal area enclosing volume $x$ in $\mathbb{R}^{n}, S^{n}$, or $\mathbb{U}^{n}$, and $A(v, w)$ denote the area of the minimal double bubble enclosing volumes $v$ and $w$.

LEMMA 4.7 (Hutchings basic estimate). Consider a minimizing double bubble enclosing volumes $v_{0}$ and $w_{0}$ on some Riemannian manifold for which a minimizer exists. Suppose further that the least-area function for two volumes, $A(v, w)$, is concave. If 
the first region has a component of volume $x>0$, then

$$
2 A\left(v_{0}, w_{0}\right) \geq A\left(w_{0}\right)+A\left(v_{0}+w_{0}\right)+\frac{v_{0}}{x} A(x) .
$$

Proof. Hutchings [10, Theorem 4.2] proves the statement for $\mathbb{R}^{n}$. The proof in the more general case is identical, except that one cannot simplify by scaling.

Proposition 4.8. Suppose that on a Riemannian manifold in which there exists a minimizer, $A(v, w)$ is concave. If $\left(v_{0} / x\right) A(x)$ is decreasing in $x$ for $x \leq v_{0}$, and for some integer $k \geq 2$,

$$
k A\left(\frac{v_{0}}{k}\right)+A\left(w_{0}\right)+A\left(v_{0}+w_{0}\right)-2 A\left(v_{0}, w_{0}\right)>0,
$$

then the region of volume $v_{0}$ has fewer than $k$ components.

PRoof. Suppose that the smallest component of the region of volume $v_{0}$ has volume $x$; the region thus has at most $v_{0} / x$ components. By our concavity assumption, we can apply Lemma 4.7 to find

$$
\frac{v_{0}}{x} A(x) \leq 2 A\left(v_{0}, w_{0}\right)-A\left(w_{0}\right)-A\left(v_{0}+w_{0}\right) .
$$

If the left-hand side of (4.4) is decreasing in $x$ for $x \leq v_{0}$, and $k A\left(v_{0} / k\right)>2 A\left(v_{0}, w_{0}\right)-$ $A\left(w_{0}\right)-A\left(v_{0}+w_{0}\right)$ for some $k$, then (4.4) is false whenever $x \leq v_{0} / k$. We conclude that the region of volume $v_{0}$ has no component of volume $x$ or smaller, so the region has fewer than $k$ components.

REMARK 4.9. Since the area of a minimal enclosure of two volumes is no greater than the area of the standard double bubble enclosing those two volumes, we can substitute the area of the standard double bubble for the area of the minimal enclosure in the left-hand side of (4.3). Thus from now on, we will use $A(v, v)$ to denote the area of the standard double bubble enclosing two volumes $v$.

LEMMA 4.10. In $\mathbb{R}^{n}, S^{n}$, and $\mathbb{\boxplus}^{n},(v / x) A(x)$ is decreasing in $x$ for all $x$ and $v$.

Proof. Since $v$ is a constant, we only need to show that the area to volume ratio for a sphere decreases as the sphere grows. In $\mathbb{R}^{n}$, this is obvious, since $A(r) / V(r)=n / r$. For $S^{n}$ and $\mathbb{a}^{n}$, we see from Remark 3.3 that $d A / d V$ is decreasing for spheres. Thus the area of a sphere as a function of volume is concave, and $A / V$ is decreasing.

Proposition 4.11. In $\mathbb{R}^{n}, S^{n}$, and $\mathbb{q}^{n}$, a minimizing double bubble has finitely many components.

Proof. By Proposition 4.8 and Lemma 4.10, we have that when (4.3) holds, then the region of volume $v_{0}$ has fewer than $k$ components. We claim that $k A\left(v_{0} / k\right)$ increases without bound as $k$ increases. This suffices to prove the proposition, for then there is some $k$ such that (4.3) is true, since all other terms remain constant.

We let $k=v_{0} / x$, and let $x$ approach zero. The term we are considering becomes $\left(v_{0} / x\right) A(x)$. Let $x=V(r)$. Since $v_{0}$ is a constant, we only need to show that $A(r) / V(r)$ increases without bound as $r$ gets small. Denote the area and volume functions for $\mathbb{R}^{n}$ 
by $A_{e}(r)$ and $V_{e}(r)$. Since $A_{e}(r) / V_{e}(r)=n / r$, the ratio obviously increases without bound. For $S^{n}$, denote the area and volume functions by $A_{s}(r)$ and $V_{s}(r)$. Then

$$
\frac{A_{s}(r)}{V_{s}(r)}=\frac{n}{r} \cdot \frac{A_{s}(r)}{A_{e}(r)} \cdot \frac{V_{e}(r)}{V_{s}(r)}
$$

Since $S^{n}$ is locally Euclidean, for small enough volumes we can make the ratios $A_{s}(r) / A_{e}(r)$ and $V_{e}(r) / V_{s}(r)$ arbitrarily close to 1 , and since $n / r$ increases without bound, $A_{s}(r) / V_{s}(r)$ does as well. The proof for $\mathbb{M}^{n}$ is identical.

5. Component bounds for equal-volume double bubbles in $S^{3}$ and $\mathbb{t}^{3}$. Propositions 5.1 and 5.2 show that both regions are connected in most area-minimizing double bubbles in $S^{3}$ and $\mathbb{H}^{3}$ enclosing two equal volumes.

Proposition 5.1. In an area-minimizing double bubble enclosing two equal volumes in $S^{3}$, each enclosed region has only one component. When the volume of the exterior is at least 10 percent of the total volume of $S^{3}$, the exterior also has only one component.

Proposition 5.2. In an area-minimizing double bubble enclosing two equal volumes in $\mathbb{\boxplus}^{3}$, each enclosed region has only one component.

The proofs appear at the end of Sections 5.1 and 5.2.

Discussion. By considering only double bubbles enclosing two equal volumes, we eliminate one degree of freedom. We will show that in both spaces, both of the regions of equal volume are connected. We will also show that in $S^{3}$, the exterior is connected when its volume is at least 10 percent of the total volume of $S^{3}$. (In $\mathbb{t}^{3}$, the exterior is always connected by Corollary 4.5.)

Let $A(v)$ denote the area of a sphere enclosing volume $v$ and $A(v, v)$ denote the area of a standard double bubble enclosing two equal volumes $v$. By Proposition 4.8, to show that the number of components of each region of an equal-volume double bubble is less than two, it suffices to show that the quantity

$$
F(v)=2 A\left(\frac{v}{2}\right)+A(v)+A(2 v)-2 A(v, v)
$$

is greater than zero.

In $\mathbb{R}^{3}$, we can solve for $F(v)$ explicitly, and see that it is equal to some positive constant times $v^{2 / 3}$, which is positive for positive $v$. Thus both regions of an equal-volume double bubble in $\mathbb{R}^{3}$ are connected. In spherical space and hyperbolic space, however, we cannot find an explicit expression for $F(v)$. We thus use a different method. For volumes near zero, we show that $F(v)$ is close enough to the Euclidean $F(v)$ so that the derivative $F^{\prime}(v)$ must be positive, which guarantees that $F(v)$ is positive in some neighborhood around zero. We then start at the edge of this neighborhood and use a lower bound on the derivative $F^{\prime}(v)$ to break up the positive $v$-axis into intervals and show that $F(v)$ is positive on each. This method relies on our ability to use a computer to find values of $F(v)$ with high precision. For large volumes in $\mathbb{R}^{3}$, we use asymptotic analysis to show that $F(v)$ is always positive. 


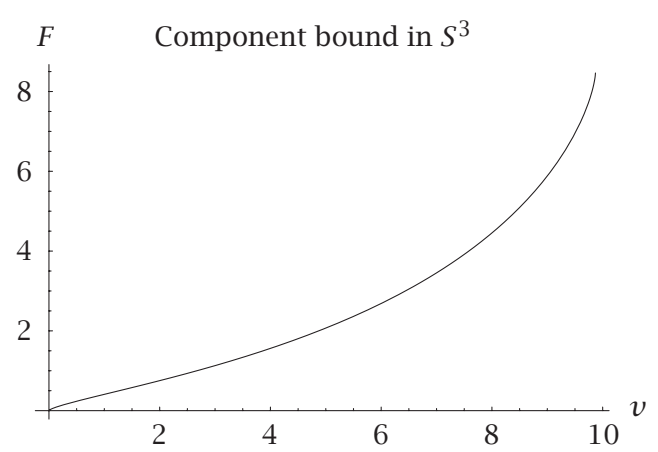

(a)

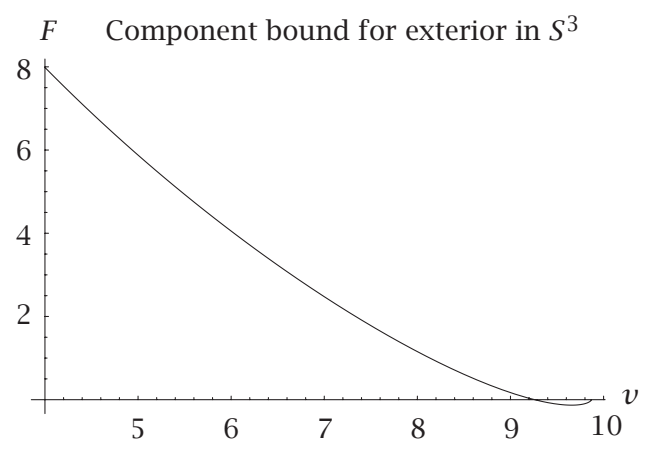

(b)

FIGURE 5.1. (a) A Mathematica plot of $F_{S}(v)$ for $v \in\left[0, \pi^{2}\right]$ suggests that $F_{\mathcal{S}}(v)$ is positive in this interval, and thus each region of a double bubble enclosing equal volumes has one component. (b) A Mathematica plot of $\widehat{F}_{S}(v)$ for $v \in\left[4, \pi^{2}\right]$ suggests that $\hat{F}_{\mathcal{S}}(v)$ is positive for $v<9$, and thus the exterior of an equal-volume double bubble is connected when it is at least 10 percent of the total volume of $S^{3}$.

5.1. Proof of component bounds for equal-volume double bubbles in $S^{3}$. Let $A_{s}(v)$ be the area of a sphere in $S^{3}$ of volume $v$, and let $A_{s}(v, v)$ be the area of the standard double bubble in $S^{3}$ enclosing two equal volumes $v$. Define the functions $F_{S}(v)$ and $\hat{F}_{S}(v)$ as follows:

$$
\begin{aligned}
& F_{S}(v)=2 A_{s}\left(\frac{v}{2}\right)+A_{s}(v)+A_{s}(2 v)-2 A_{s}(v, v), \\
& \hat{F}_{S}(v)=2 A_{s}\left(\pi^{2}-v\right)+A_{s}(v)+A_{S}\left(2 \pi^{2}-v\right)-2 A_{s}(v, v) .
\end{aligned}
$$

By Proposition 4.8 and Lemma 4.10, if $F_{S}(v)>0$ for some $v$, then the two enclosed regions of a double bubble enclosing two equal volumes $v$ are connected. Again by Proposition 4.8, Lemma 4.10, and the fact that a minimal enclosure of two volumes $v$ in $S^{3}$ must also be a minimal enclosure of volumes $2 \pi^{2}-2 v$ and $v$, if $\hat{F}_{\mathcal{S}}(v)>0$ for some $v$, then the region exterior to a double bubble enclosing two equal volumes $v$ is connected. 
Mathematica plots of $F_{S}(v)$ and $\hat{F}_{S}(v)$ for $v \in\left[0, \pi^{2}\right]$ (see Figure 5.1) suggest that $F_{S}(v)$ is positive on this whole interval and that $\widehat{F}_{S}(v)$ is positive for $v<9$. Thus the plots suggest that for a double bubble enclosing two equal volumes in $S^{3}$, both regions of equal volume are connected for all volumes, and the exterior is connected when its volume is at least 10 percent of $S^{3}$. However, the plots are not rigorous proofs, as $F_{S}$ or $\widehat{F}_{S}$ may behave badly between plotted points.

We first find a neighborhood around zero in which $F_{S}(v)$ must be positive. Our method makes use of the fact that for very small volumes, $S^{3}$ looks nearly flat. Thus the area of a sphere or double bubble of radius $r$ in $S^{3}$ approaches that of a sphere or double bubble of the same radius in $\mathbb{R}^{3}$. The following two lemmas give precise formulations of this statement.

LEMMA 5.3. Let $A_{e}(v)$ and $A_{s}(v)$ be the surface area of spheres of volume $v$ in $\mathbb{R}^{3}$ and $S^{3}$, respectively. Let $r_{e}$ and $r_{s}$ be the radii of these spheres in $\mathbb{R}^{3}$ and $S^{3}$, respectively. Then for $r_{s}<1 / 2$,

$$
\left|A_{e}^{\prime}(v)-A_{s}^{\prime}(v)\right|<1.6 r_{s}
$$

Proof. By Remark 3.3,

$$
A_{e}^{\prime}(v)-A_{s}^{\prime}(v)=\frac{2}{r_{e}}-2 \cot r_{s}
$$

Since the volume of a sphere increases less rapidly with radius in $S^{3}$ than in $\mathbb{R}^{3}, r_{s}>r_{e}$. Since $\cot x$ decreases in $x$ on $(0, \pi), A_{e}^{\prime}(v)-A_{s}^{\prime}(v)$ is obviously positive for all $v$. It remains to find an upper bound on this quantity for small $v$.

By Remark 3.2,

$$
v=\frac{4}{3} \pi r_{e}^{3}=\pi\left(2 r_{s}-\sin 2 r_{s}\right)
$$

and thus

$$
r_{e}=\left[\frac{3}{4}\left(2 r_{s}-\sin 2 r_{s}\right)\right]^{1 / 3}
$$

For $x<1, \sin x<x-(1 / 3$ ! $) x^{3}+\left(1 / 5\right.$ !) $x^{5}$, so for $r_{s}<1 / 2$ we have

$$
2 r_{s}-\sin 2 r_{s}>\frac{4}{3} r_{s}^{3}-\frac{4}{15} r_{s}^{5}
$$

Thus for $r_{s}<1 / 2$,

$$
r_{e}>\left[\frac{3}{4}\left(\frac{4}{3} r_{s}^{3}-\frac{4}{15} r_{s}^{5}\right)\right]^{1 / 3}
$$


and substituting this expression into (5.5) gives

$$
\begin{aligned}
\left|A_{e}^{\prime}(v)-A_{s}^{\prime}(v)\right| & <\frac{2}{r_{s}}\left(1-\frac{1}{5} r_{s}^{2}\right)^{-1 / 3}-2 \cot r_{s} \\
& <\frac{2}{r_{s}}\left(1+\frac{2}{15} r_{s}^{2}\right)-2 \cot r_{s}
\end{aligned}
$$

since $(1-x)^{-1 / 3}<1+(2 / 3) x$ for $x<1 / 2$. For $x<1 / 2$, $\tan x<x+(2 / 3) x^{3}$, so

$$
\begin{aligned}
\left|A_{e}^{\prime}(v)-A_{s}^{\prime}(v)\right| & <\frac{2}{r_{s}}\left(1+\frac{2}{15} r_{s}^{2}\right)-\frac{2}{r_{s}+(2 / 3) r_{s}^{3}} \\
& <\frac{2}{r_{s}}\left(1+\frac{2}{15} r_{s}^{2}\right)-\frac{2}{r_{s}}\left(1-\frac{2}{3} r_{s}^{2}\right),
\end{aligned}
$$

since $(1+x)^{-1}>1-x$ for $x<1 / 2$. Thus we have, for $r_{s}<1 / 2$,

$$
\left|A_{e}^{\prime}(v)-A_{s}^{\prime}(v)\right|<\frac{8}{5} r_{s}
$$

LEMMA 5.4. Let $A_{e}(v, v)$ and $A_{s}(v, v)$ be the surface area of standard double bubbles enclosing two equal volumes $v$ in $\mathbb{R}^{3}$ and $S^{3}$, respectively. Let $r_{e}$ and $r_{s}$ be the radii of these bubbles in $\mathbb{R}^{3}$ and $S^{3}$, respectively. Then for $r_{s}<1 / 2$,

$$
\left|A_{e}^{\prime}(v, v)-A_{s}^{\prime}(v, v)\right|<3.7 r_{s} .
$$

Proof. From Remark 3.7, we have

$$
A_{e}^{\prime}(v, v)-A_{s}^{\prime}(v, v)=\frac{4}{r_{e}}-4 \cot r_{s}
$$

Since the volume of a standard equal-volume double bubble increases less rapidly with radius in $S^{3}$ than in $\mathbb{R}^{3}, r_{s}>r_{e}$. Since cot $x$ decreases in $x$ on $(0, \pi), A_{e}^{\prime}(v, v)-A_{s}^{\prime}(v, v)$ is obviously positive for all $v$. It remains to find an upper bound on this quantity for small $v$.

From Proposition 3.5 and Remark 3.6, we have

$$
\begin{aligned}
v=\frac{9}{8} \pi r_{e}^{3}= & \frac{\pi}{2}\left(2 r_{s}-\sin 2 r_{s}\right)\left(1+\frac{\sqrt{2} \cos r_{s}}{\sqrt{7+\cos 2 r_{s}}}\right) \\
& +\pi\left(\tan ^{-1}\left(\frac{\sqrt{2} \sin r_{s}}{\sqrt{7+\cos 2 r_{s}}}\right)-\frac{\sqrt{2} r_{s} \cos r_{s}}{\sqrt{7+\cos 2 r_{s}}}\right),
\end{aligned}
$$

so

$$
\begin{aligned}
r_{e}=[ & \frac{4}{9}\left(2 r_{s}-\sin 2 r_{s}\right)\left(1+\frac{\sqrt{2} \cos r_{s}}{\sqrt{7+\cos 2 r_{s}}}\right) \\
& \left.+\frac{8}{9}\left(\tan ^{-1}\left(\frac{\sqrt{2} \sin r_{s}}{\sqrt{7+\cos 2 r_{s}}}\right)-\frac{\sqrt{2} r_{s} \cos r_{s}}{\sqrt{7+\cos 2 r_{s}}}\right)\right]^{1 / 3} .
\end{aligned}
$$


We use power series expansions to find upper bounds on each term of this formula. We begin with the second half of the formula. For $x<\pi / 2, \tan ^{-1} x>x-(1 / 3) x^{3}$, so

$$
\begin{aligned}
r_{e}> & {\left[\frac{4}{9}\left(2 r_{s}-\sin 2 r_{s}\right)\left(1+\frac{\sqrt{2} \cos r_{s}}{\sqrt{7+\cos 2 r_{s}}}\right)\right.} \\
& \left.+\frac{8}{9}\left(\frac{\sqrt{2}}{\sqrt{7+\cos 2 r_{s}}}\left(\sin r_{s}-r_{s} \cos r_{s}\right)-\frac{1}{3}\left(\frac{\sqrt{2} \sin r_{s}}{\sqrt{7+\cos 2 r_{s}}}\right)^{3}\right)\right]^{1 / 3} .
\end{aligned}
$$

For $x<1 / 2, \cos 2 x>1-2 x^{2}$ and $(1-x)^{-1 / 2}<1+x$, so

$$
\frac{1}{\sqrt{7+\cos 2 r_{s}}}<\frac{1}{\sqrt{8}}\left(1-\frac{1}{4} r_{s}^{2}\right)^{-1 / 2}<\frac{1}{\sqrt{8}}\left(1+\frac{1}{4} r_{s}^{2}\right) .
$$

In addition, $\sin x<x$ for $x>0$, so we have

$$
\frac{1}{3}\left(\frac{\sqrt{2} \sin r_{s}}{\sqrt{7+\cos 2 r_{s}}}\right)^{3}<\frac{1}{3}\left(\frac{r_{s}}{2}\left(1+\frac{1}{4} r_{s}^{2}\right)\right)^{3}<\frac{1}{24} r_{s}^{3}+\frac{1}{24} r_{s}^{5}
$$

To deal with the term $\left(\sqrt{2} / \sqrt{7+\cos 2 r_{s}}\right)\left(\sin r_{s}-r_{s} \cos r_{s}\right)$, we note that for all $r_{s}$,

$$
\frac{\sqrt{2}}{\sqrt{7+\cos 2 r_{s}}} \geq \frac{1}{2}
$$

and for $r_{s}<1$,

$$
\sin r_{s}-r_{s} \cos r_{s}>\frac{1}{3} r_{s}^{3}-\frac{1}{30} r_{s}^{5}
$$

Substituting these approximations into (5.16) gives

$$
\begin{aligned}
r_{e}> & {\left[\frac{4}{9}\left(2 r_{s}-\sin 2 r_{s}\right)\left(1+\frac{1}{2} \cos r_{s}\right)\right.} \\
& \left.+\frac{8}{9}\left(\frac{1}{2}\left(\frac{1}{3} r_{s}^{3}-\frac{1}{30} r_{s}^{5}\right)-\left(\frac{1}{24} r_{s}^{3}+\frac{1}{24} r_{s}^{5}\right)\right)\right]^{1 / 3} .
\end{aligned}
$$

We now attack the first half of the expression. By the Taylor series expansions for $\sin x$ and $\cos x$ we have, for $r_{s}<1$,

$$
2 r_{s}-\sin 2 r_{s}>\frac{4}{3} r_{s}^{3}-\frac{4}{15} r_{s}^{5}, \quad \cos r_{s}>1-\frac{1}{2} r_{s}^{2}
$$

We now have a polynomial approximation for $r_{e}$ that holds whenever $r_{s}<1 / 2$

$$
\begin{aligned}
r_{e}> & {\left[\frac{4}{9}\left(\frac{4}{3} r_{s}^{3}-\frac{4}{15} r_{s}^{5}\right)\left(1+\frac{1}{2}-\frac{1}{4} r_{s}^{2}\right)\right.} \\
& \left.+\frac{8}{9}\left(\frac{1}{2}\left(\frac{1}{3} r_{s}^{3}-\frac{1}{30} r_{s}^{5}\right)-\left(\frac{1}{24} r_{s}^{3}+\frac{1}{24} r_{s}^{5}\right)\right)\right]^{1 / 3} \\
= & \left(r_{s}^{3}-\frac{17}{45} r_{s}^{5}+\frac{4}{135} r_{s}^{7}\right)^{1 / 3} \\
> & \left(r_{s}^{3}-\frac{17}{45} r_{s}^{5}\right)^{1 / 3} .
\end{aligned}
$$


Using this approximation for $r_{e}$, we have, for $r_{s}<1 / 2$,

$$
\begin{aligned}
\left|A_{e}^{\prime}(v, v)-A_{s}^{\prime}(v, v)\right| & <\frac{4}{r_{s}}\left(1-\frac{17}{45} r_{s}^{2}\right)^{-1 / 3}-4 \cot r_{s} \\
& <\frac{4}{r_{s}}\left(1+\frac{34}{135} r_{s}^{2}\right)-4 \cot r_{s}
\end{aligned}
$$

since $(1-x)^{-1 / 3}<1+(2 / 3) x$ for $x<1 / 2$. For $x<1 / 2$, $\tan x<x+(2 / 3) x^{3}$, so

$$
\begin{aligned}
\left|A_{e}^{\prime}(v, v)-A_{s}^{\prime}(v, v)\right| & <\frac{4}{r_{s}}\left(1+\frac{34}{135} r_{s}^{2}\right)-\frac{4}{r_{s}+(2 / 3) r_{s}^{3}} \\
& <\frac{4}{r_{s}}\left(1+\frac{34}{135} r_{s}^{2}\right)-\frac{4}{r_{s}}\left(1-\frac{2}{3} r_{s}^{2}\right)
\end{aligned}
$$

since $(1+x)^{-1}>1-x$ for $x<1 / 2$. Thus we have, for $r_{s}<1 / 2$,

$$
\left|A_{e}^{\prime}(v, v)-A_{s}^{\prime}(v, v)\right|<\frac{496}{135} r_{s}<3.7 r_{s}
$$

We now use these two lemmas to show that $F_{S}$ is positive for small $v$.

Proposition 5.5. Let $F_{\mathcal{S}}(v)$ be defined as in (5.2). Then for $0<v \leq 0.002, F_{\mathcal{S}}(v)>0$.

Proof. By (5.2), we have

$$
F_{s}^{\prime}(v)=A_{s}^{\prime}\left(\frac{v}{2}\right)+A_{s}^{\prime}(v)+2 A_{s}^{\prime}(2 v)-2 A_{s}^{\prime}(v, v)
$$

in both $\mathbb{R}^{3}$ and $S^{3}$. By Lemmas 5.3 and 5.4 , we have

$$
\begin{aligned}
\left|F_{e}^{\prime}(v)-F_{s}^{\prime}(v)\right| & <1.6 r_{s}\left(\frac{v}{2}\right)+1.6 r_{s}(v)+3.2 r_{s}(2 v)+7.4 r_{s}(v, v) \\
& <13.8 r_{s}(2 v)
\end{aligned}
$$

when all of the $r_{s}$ are less than $1 / 2$. The largest of the four $r_{s}$ is obviously $r_{s}(2 v)$, so we can replace all of the others with that one.

Direct computation from the volume formula (Remark 3.2) shows that the volume of a sphere in $S^{3}$ of radius 0.1 is greater than 0.004 , so if $v \leq 0.002$, then $r_{s}(2 v)<0.1$ and $\left|F_{e}^{\prime}(v)-F_{s}^{\prime}(v)\right|<1.38$.

In $\mathbb{R}^{3}$, we can solve the area and volume formulae to find that $F_{e}(v)=c v^{2 / 3}$, where

$$
c=(36 \pi)^{1 / 3}\left(1+2^{1 / 3}+2^{2 / 3}\right)-2 \cdot 3^{5 / 3} \pi^{1 / 3} \approx 0.327 .
$$

Thus $F_{e}^{\prime}(v)$ is equal to $(2 / 3) c v^{-1 / 3}$, which is greater than 1.72 when $v=0.002$. Since $F_{e}^{\prime}(v)$ is obviously decreasing in $v, F_{e}^{\prime}(v)>1.72$ for $v \leq 0.002$.

We conclude that $F_{s}^{\prime}(v)>0$ for $v \leq 0.002$. Since $F_{s}(0)$ is obviously zero, $F_{s}(v)$ must be positive when $0<v \leq 0.002$. 
Next we calculate a lower bound on the derivatives of $F_{S}(v)$ and $\widehat{F}_{\mathcal{S}}(v)$ and use a computer to show that these functions are positive on the required intervals.

LEMMA 5.6. Let $F_{S}(v)$ and $\hat{F}_{S}(v)$ be defined as in (5.2) and (5.3). Then for $v \in$ $\left(0,(4 / 5) \pi^{2}\right]$,

$$
F_{S}^{\prime}(v)>-2.5-8 \cot \left(\frac{8 v}{9 \pi}\right)^{1 / 3}
$$

and for $v \in\left(0,(9 / 10) \pi^{2}\right]$,

$$
\hat{F}_{s}^{\prime}(v)>-3-8 \cot \left(\frac{8 v}{9 \pi}\right)^{1 / 3}
$$

Proof. From (5.2), we have

$$
F_{s}^{\prime}(v)=A_{s}^{\prime}\left(\frac{v}{2}\right)+A_{s}^{\prime}(v)+2 A_{s}^{\prime}(2 v)-2 A_{s}^{\prime}(v, v)
$$

By Remark 3.3, $A_{s}^{\prime}(v)=2 \cot r_{s}(v)$, where $r_{s}(v)$ is the radius of a sphere of volume $v$. By Remark 3.7, $A_{s}^{\prime}(v, v)=4 \cot r_{s}(v, v)$, where $r_{s}(v, v)$ is the radius of one half of the double bubble enclosing two equal volumes $v$. We thus have

$$
F_{s}^{\prime}(v)=2 \cot r_{s}\left(\frac{v}{2}\right)+2 \cot r_{s}(v)+4 \cot r_{s}(2 v)-8 \cot r_{s}(v, v)
$$

Similarly, from (5.3) and the fact that $A_{s}\left(2 \pi^{2}-2 v, v\right)=A_{s}(v, v)$, we have

$$
\hat{F}_{s}^{\prime}(v)=-2 \cot r_{s}\left(\pi^{2}-v\right)+4 \cot r_{s}(v)-2 \cot r_{s}\left(2 \pi^{2}-v\right)-8 \cot r_{s}(v, v) .
$$

Since a sphere of radius $\pi / 2$ encloses a volume of $\pi^{2}$, or half of $S^{3}$, and since $r_{s}(v)$ is increasing in $v$ and $\cot x>0$ for $x \in(0, \pi / 2)$, the first two terms of $F_{s}^{\prime}$ and the middle two terms of $\hat{F}_{s}^{\prime}$ are always positive. For the other spherical term in $F_{s}^{\prime}$, we note that a sphere of radius 2.1 has volume greater than $(8 / 5) \pi^{2}$, so in the interval we are considering, the third term of $F_{s}^{\prime}$ is greater than $4 \cot 2.1$ (since $\cot x$ is decreasing in $x$ ), which is greater than -2.5 . In addition, a sphere of radius 0.6 has volume less than $(1 / 10) \pi^{2}$, so in the interval we are considering, the first term of $\hat{F}_{s}^{\prime}$ is greater than $-2 \cot 0.6$, which is greater than -3 .

Since an equal-volume double bubble of radius $r$ has less volume in $S^{3}$ than in $\mathbb{R}^{3}$, and $r_{s}(v, v)$ is increasing in $v$, by Remark 3.6, $r_{s}(v, v)>r_{e}(v, v)=(8 v / 9 \pi)^{1 / 3}$. This suffices to put a lower bound on the fourth term of both equations. We thus have, for $v \leq(4 / 5) \pi^{2}$,

$$
F_{S}^{\prime}(v)>-2.5-8 \cot \left(\frac{8 v}{9 \pi}\right)^{1 / 3}
$$

and for $v \leq(9 / 10) \pi^{2}$,

$$
\hat{F}_{S}^{\prime}(v)>-3-8 \cot \left(\frac{8 v}{9 \pi}\right)^{1 / 3} .
$$

It is clear that these bounds are increasing in $v$. 
We will also need the following algebraic properties of the volume function for double bubbles.

LEMMA 5.7. Let $V_{s}(r, r)$ be the volume of one half of an equal-volume double bubble in $S^{3}$, and let $V_{s}^{\prime}(r, r)$ and $V_{s}^{\prime \prime}(r, r)$ be the first and second derivatives of $V_{s}$ with respect to $r$, respectively. Then

(1) $V_{s}^{\prime}(r, r) \geq \pi \sin ^{2} r(2-\sqrt{3})$ for $r \in[0, \pi]$,

(2) $V_{s}^{\prime \prime}(r, r) \geq 2 \pi \sin r$ for $r \in[0, \pi / 4]$.

Proof. Differentiating the formula for $V_{s}(r, r)$ given in Proposition 3.5 gives

$$
\begin{aligned}
V_{s}^{\prime}(r, r) & =2 \pi \sin ^{2} r+\frac{6 \sqrt{2} \pi \sin ^{2} r \cos r}{(7+\cos 2 r)^{3 / 2}}+\frac{2 \sqrt{2} \pi \sin ^{2} r \cos r}{(7+\cos 2 r)^{1 / 2}} \\
& \geq \pi \sin ^{2} r\left(2-\frac{6 \sqrt{2}}{6^{3 / 2}}-\frac{2 \sqrt{2}}{6^{1 / 2}}\right) \\
& \geq \pi \sin ^{2} r(2-\sqrt{3}) .
\end{aligned}
$$

Differentiating again gives

$$
\begin{aligned}
V_{s}^{\prime \prime}(r, r)= & -24 \pi \frac{\sqrt{2} \sin ^{3} r}{(7+\cos 2 r)^{3 / 2}}+4 \pi \sin r \cos r\left(1+\frac{\sqrt{2} \cos r}{(7+\cos 2 r)^{1 / 2}}\right) \\
& +3 \sqrt{2} \pi \frac{24 \sin r \cos ^{2} r-8 \cos ^{4} r \sin r+16 \sin r-4 \sin ^{3} r}{(7+\cos 2 r)^{5 / 2}}
\end{aligned}
$$

For $r \in[0, \pi / 4]$, we have

$$
\begin{aligned}
V_{s}^{\prime \prime}(r, r) \geq \sin r( & -24 \pi \frac{\sqrt{2}(1 / \sqrt{2})^{2}}{7^{3 / 2}}+4 \pi \frac{1}{\sqrt{2}}\left(1+\frac{\sqrt{2}(1 / \sqrt{2})}{8^{1 / 2}}\right) \\
& \left.+3 \sqrt{2} \pi \frac{24(1 / \sqrt{2})^{2}}{8^{5 / 2}}-3 \sqrt{2} \pi \frac{8+4(1 / \sqrt{2})^{2}}{7^{5 / 2}}\right) .
\end{aligned}
$$

Simplifying the right-hand side gives

$$
\begin{aligned}
V_{s}^{\prime \prime}(r, r) & \geq \pi \sin r\left(-\frac{12 \sqrt{2}}{7 \sqrt{7}}+2 \sqrt{2}+1+\frac{9}{32}-\frac{30 \sqrt{2}}{49 \sqrt{7}}\right) \\
& >2 \pi \sin r .
\end{aligned}
$$

Proposition 5.8. Let $F_{S}(v)$ and $\widehat{F}_{S}(v)$ be defined as in (5.2) and (5.3). Then for $v \in\left[0.002,(4 / 5) \pi^{2}\right], F(v)>0$, and for $v \in\left[(1 / 2) \pi^{2},(9 / 10) \pi^{2}\right], \hat{F}_{\mathcal{S}}(v)>0$.

Proof. Our method is as follows: given an interval $\left[v_{0}, v_{f}\right]$, we find a $\delta_{0}$ such that $F_{S}(v)>0$ for $v \in\left[v_{0}, v_{0}+\delta_{0}\right]$. We then let $v_{1}=v_{0}+\delta_{0}$ and repeat the process until $v_{n}+\delta_{n}>v_{f}$.

We now consider a single iteration of this process, beginning at $v_{0}$. Let $G_{s}(v)$ be our lower bound on the derivative $F_{s}^{\prime}(v)$ (the right-hand side of (5.31)). Since $G_{s}(v)$ is increasing in $v$, for all $v \geq v_{0}, F_{S}^{\prime}(v)>G_{s}\left(v_{0}\right)$ and thus $F_{S}(v)>F_{S}\left(v_{0}\right)+G_{s}\left(v_{0}\right)(v-$ $\left.v_{0}\right)$. Thus if $F_{S}\left(v_{0}\right)>0$ and $G_{s}\left(v_{0}\right)<0$, then $F_{S}(v)>0$ for all $v$ in the interval $\left[v_{0}, v_{0}-\right.$ $\left.F_{s}\left(v_{0}\right) / G_{\mathcal{S}}\left(v_{0}\right)\right]$. If $G_{s}\left(v_{0}\right) \geq 0$ then $F_{\mathcal{S}}(v)>0$ for all $v>v_{0}$. 
Since we cannot compute $F_{\mathcal{S}}(v)$ explicitly, we make use of Mathematica's equationsolving abilities. For a volume $v$, Mathematica uses Newton's method to find, with 10 digits of accuracy and 25 digits of internal working precision, the radii $r_{s}(v / 2)$, $r_{s}(v)$, and $r_{s}(2 v)$ of spheres of volumes $v / 2, v$, and $2 v$, respectively, as well as the radius $r_{s}(v, v)$ of the standard double bubble enclosing two volumes $v$. We must verify that Newton's method gives a number near the actual solution and not a spurious or negative number. However, since all of the functions we consider are increasing for positive volumes, we need only check that the number returned by Newton's method is between zero and $\pi$. Once this is verified, since Mathematica's internal calculations have 16 digits of precision and no radius is ever greater than $\pi$, we need only consider how much the error from Newton's method is magnified when the calculated radii are plugged into the area formulae to calculate $F_{S}(v)$.

The statement that Mathematica calculates the solution $x=x_{0}$ to the equation $f(x)=y_{0}$ with $n$ digits of accuracy means that

$$
\left|f\left(x_{0}\right)-y_{0}\right|<10^{-n}
$$

Let $\epsilon=10^{-n}$ (where for our purposes $n$ is 10) and let $\Delta>0$ be a lower bound on $\left|f^{\prime}(x)\right|$ over all $x$ in some interval $[a, b]$ that contains the exact solution $\tilde{x}$ and the calculated solution $x_{0}$ :

$$
\Delta \leq \min _{x \in[a, b]}\left|f^{\prime}(x)\right|
$$

Then we have

$$
\left|\tilde{x}-x_{0}\right|<\Delta^{-1} \epsilon
$$

To find the error in the calculated value of $F_{s}(v)=2 A_{s}(v / 2)+A_{s}(v)+A_{s}(2 v)-$ $2 A_{s}(v, v)$, we will consider each term separately. We first consider the terms that are areas of spheres. In this case, the function $f$ in which we are interested is $V_{s}(r)$, the volume of a sphere of radius $r$, given by $V_{s}(r)=\pi(2 r-\sin 2 r)$. (See Remark 3.2.) Since the derivative $V_{s}^{\prime}(r)$ is increasing on $(0, \pi / 2)$ and decreasing on $(\pi / 2, \pi)$, we may take $\Delta$ to be any number smaller than the values of the derivative at the smallest and largest $r$ we are considering, which are $r(v / 2)$ for $v=0.002$ and $r(2 v)$ for $v=$ $(9 / 10) \pi^{2}$, respectively. The value $r=0.062$ gives $V_{s}(r)<0.001-\epsilon$ and the derivative $V_{s}^{\prime}(r)>0.048$, while the value $r=2.33$ gives $V_{s}(r)>(9 / 5) \pi^{2}+\epsilon$ and $V_{s}^{\prime}(r)>6$. We may thus bound $V_{s}^{\prime}(r)$ by $\Delta_{1}=0.048$.

If we want to calculate $A_{s}(r)$, the area of a sphere of radius $r$, then we have

$$
\left|A_{s}(r)-A_{s}\left(r_{0}\right)\right|<\Delta_{1}^{-1} \epsilon\left(\sup _{r \in\left(r_{0}-\Delta_{1}^{-1} \epsilon, r_{0}+\Delta_{1}^{-1} \epsilon\right)}\left|A_{s}^{\prime}(r)\right|\right)
$$

for $r \in\left(r_{0}-\Delta_{1}^{-1} \epsilon, r_{0}+\Delta_{1}^{-1} \epsilon\right)$. By Remark 3.1, $A_{s}^{\prime}(r)=4 \pi \sin 2 r$, so $\left|A_{s}^{\prime}(r)\right| \leq 4 \pi$ for all $r$. Setting $\Delta_{1}$ equal to 0.048 , we conclude that the error in the calculated value of $A_{s}(r)$ is less than $84 \pi \epsilon$. 
To bound the error on the term of $F_{s}(v)$ that is the area of the double bubble, we first bound the derivative $V_{s}^{\prime}(r, r)$ (where $V_{s}(r, r)$ is the volume of one half of an equalvolume double bubble of radius $r$ ). The values of $r$ in which we are interested are those for which $0.002-\epsilon \leq V_{s}(r, r) \leq(9 / 10) \pi^{2}+\epsilon$. Since $r=0.082$ gives $V_{s}(r, r)<$ $0.002-\epsilon, r=2.07$ gives $V_{s}(r, r)>(9 / 10) \pi^{2}+\epsilon$, and $V_{s}$ is increasing in $r$ (by part (1) of Lemma 5.7), these $r$ fall within the range [0.082,2.07]. By part (2) of Lemma 5.7, $V_{s}^{\prime}(r, r)$ is increasing on $(0, \pi / 4]$, so

$$
\min _{r \in[0.082, \pi / 4]} V_{s}^{\prime}(r, r)=V_{s}^{\prime}(0.082,0.082)>0.071,
$$

while by part (1) of the same lemma,

$$
\begin{aligned}
\min _{r \in[\pi / 4,2.07]} V_{s}^{\prime}(r, r) & \geq \min _{r \in[\pi / 4,2.07]}\left(\pi \sin ^{2} r(2-\sqrt{3})\right) \\
& \geq \pi(2-\sqrt{3})\left(\min \left\{\sin ^{2} \frac{\pi}{4}, \sin ^{2} 2.07\right\}\right) \\
& >0.42 .
\end{aligned}
$$

We may thus bound the derivative $V_{s}^{\prime}(r, r)$ by $\Delta_{2}=0.071$.

We now take the derivative of the area formula in Proposition 3.4

$$
A_{s}^{\prime}(r, r)=4 \pi \sin 2 r \pm 4 \pi \sin ^{2} r \frac{d \zeta}{d r} \pm 4 \pi \sin 2 r \zeta \mp 4 \pi \frac{d \zeta}{d r},
$$

where $\zeta=\sqrt{2} \cos r / \sqrt{7+\cos 2 r}$. We thus have

$$
\begin{aligned}
A_{s}^{\prime}(r, r)= & 4 \pi \sin 2 r \mp 4 \pi \cos ^{2} r\left(-\frac{6 \sqrt{2} \sin r}{(7+\cos 2 r)^{3 / 2}}\right) \\
& \pm 4 \pi \sin 2 r\left(\frac{\sqrt{2} \cos r}{\sqrt{7+\cos 2 r}}\right),
\end{aligned}
$$

and therefore

$$
\left|A_{S}^{\prime}(r, r)\right| \leq 4 \pi+\frac{8 \pi}{\sqrt{3}}
$$

By the formula analogous to (5.45) with $A_{s}(r)$ replaced by $A_{s}(r, r)$, we conclude that

$$
\left|A_{s}(r, r)-A_{s}\left(r_{0}, r_{0}\right)\right| \leq \Delta_{2}^{-1} \epsilon\left(4 \pi+\frac{8 \pi}{\sqrt{3}}\right)<122 \pi \epsilon .
$$

We add up the errors from the four terms of $F_{s}(v)$ to get the final result

$$
\left|F_{S}(r)-F_{S}\left(r_{0}\right)\right| \leq \epsilon(2(84 \pi)+84 \pi+84 \pi+2(122 \pi))<580 \pi \epsilon .
$$

We conclude that if Mathematica returns a positive value for $F_{S}\left(v_{0}\right)$, then $F_{S}(v)$ is guaranteed to be positive on the interval $\left[v_{0}, v_{0}-\left(F_{\mathcal{S}}\left(v_{0}\right)-580 \pi \epsilon\right) / G\left(v_{0}\right)\right]$.

We write a simple Mathematica program to carry out this procedure; for the program's code, see Appendix A. We enter 0.002 and $(4 / 5) \pi^{2}$ for the starting and ending values of $v$, and the program tells us that $F_{S}(v)$ is positive everywhere in between. 
We carry out the same procedure for $\hat{F}_{s}$, using the same error bound (with $\hat{G}_{s}$, the right-hand side of (5.32), in place of $G_{s}$ ) and starting and ending values of $(1 / 2) \pi^{2}$ and $(9 / 10) \pi^{2}$, and the program tells us that $\hat{F}_{S}$ is positive everywhere in between.

Proof of Proposition 5.1. By Proposition 4.8 and Lemma 4.10, the two regions of equal volume $v$ are connected if $F_{s}(v)=2 A_{s}(v / 2)+A_{s}(v)+A_{s}(2 v)-2 A_{s}(v, v)$ is greater than zero. By Propositions 5.5 and $5.8, F_{s}(v)$ is greater than zero for $v \leq$ $(4 / 5) \pi^{2}$. When $v$ is greater than $(4 / 5) \pi^{2}$, the regions of volume $v$ are more than twice as large as the exterior, so they are connected by Corollary 4.2.

Similarly, by Proposition 4.8 and Lemma 4.10, the exterior region is connected if $\hat{F}_{S}(v)=2 A_{s}\left(\pi^{2}-v\right)+A_{s}(v)+A_{s}\left(2 \pi^{2}-v\right)-2 A_{s}(v, v)$ is greater than zero. By Proposition 5.8, $\hat{F}_{S}(v)$ is greater than zero for $v \in\left[(1 / 2) \pi^{2},(9 / 10) \pi^{2}\right]$. When $v$ is less than $(1 / 2) \pi^{2}$, the exterior is more than twice as large as either of the other two regions, so it is connected by Corollary 4.2. Thus the exterior is connected when $v<(9 / 10) \pi^{2}$, that is, when the bubble's volume is less than 90 percent of the volume of $S^{3}$.

5.2. Proof of component bounds for equal-volume double bubbles in $\mathbb{\sharp}^{3}$. We now prove component bounds for equal-volume double bubbles in $\mathbb{t}^{3}$. We use the same techniques as in $S^{3}$ for small and intermediate volumes, and for large volumes we use asymptotic analysis of $F_{h}(v)$.

Let $A_{h}(v)$ be the area of a sphere in $\mathbb{M}^{3}$ of volume $v$, and let $A_{h}(v, v)$ be the area of the standard double bubble in $\mathbb{\boxplus}^{3}$ enclosing two equal volumes $v$. Define the function $F_{h}(v)$ as follows:

$$
F_{h}(v)=2 A_{h}\left(\frac{v}{2}\right)+A_{h}(v)+A_{h}(2 v)-2 A_{h}(v, v)
$$

By Proposition 4.8 and Lemma 4.10, if $F_{h}(v)>0$ for some $v$, then the two enclosed regions of a double bubble enclosing two equal volumes $v$ are connected.

Mathematica plots of $F_{h}(v)$ for $v \in\left[0,10^{6}\right]$ (see Figure 5.2) suggest that this function is positive on the range plotted, and thus both regions of a double bubble enclosing two equal volumes $v$ in $\mathbb{\boxplus}^{3}$ are connected when $v$ is less than one million. Again, the plot is not a rigorous proof, as the function $F_{h}(v)$ may behave badly between points plotted. In addition, although the function appears to approach an asymptote of 0.499 as $v$ increases, in theory it could eventually go negative.

Again, we begin by giving a precise formulations of the statement that for small volumes, $\mathbb{M}^{3}$ is nearly flat, and the area of a sphere or double bubble of radius $r$ in $\mathbb{U}^{3}$ approaches that of a sphere or double bubble of the same radius in $\mathbb{R}^{3}$.

LEMMA 5.9. Let $A_{e}(v)$ and $A_{h}(v)$ be the surface area of spheres of volume $v$ in $\mathbb{R}^{3}$ and $\mathbb{M}^{3}$, respectively. Let $r_{e}$ and $r_{h}$ be the radii of these spheres in $\mathbb{R}^{3}$ and $\mathbb{M}^{3}$, respectively. Then for $r_{h}<1 / 2$,

$$
\left|A_{h}^{\prime}(v)-A_{e}^{\prime}(v)\right|<1.6 r_{h}
$$




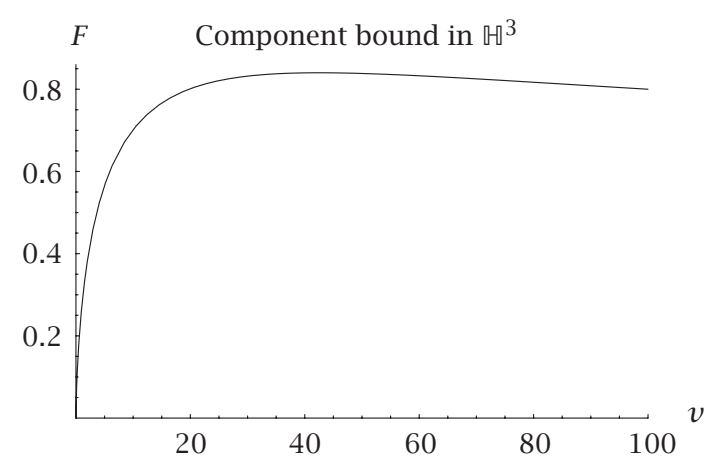

(a)

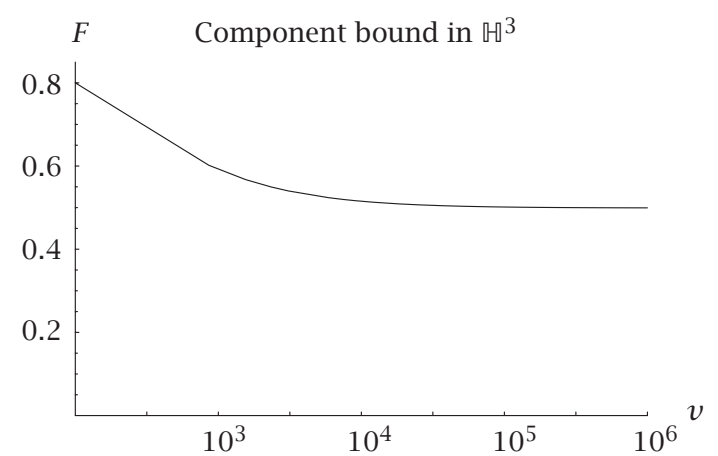

(b)

FIGURE 5.2. Plots of $F_{h}(v)$ for $v \in\left[0,10^{6}\right]$ suggest that $F_{h}(v)$ is positive in this interval, and thus each region of a double bubble enclosing equal volumes has one component. (Graph (a) plots $F_{h}(v)$ for $v \in[0,100]$, and graph (b) plots $F_{h}(v)$ for $v \in\left[100,10^{6}\right]$ on a logarithmic scale.)

Proof. By Remark 3.3,

$$
A_{h}^{\prime}(v)-A_{e}^{\prime}(v)=2 \operatorname{coth} r_{h}-\frac{2}{r_{e}}
$$

Since the volume of a sphere increases more rapidly with radius in $\mathbb{M}^{3}$ than in $\mathbb{R}^{3}$, $r_{h}<r_{e}$. Since $\operatorname{coth} x$ decreases in $x$ for $x>0, A_{h}^{\prime}(v)-A_{e}^{\prime}(v)$ is obviously positive for all $v$. It remains to find an upper bound on this quantity for small $v$. By Remark 3.2,

$$
v=\frac{4}{3} \pi r_{e}^{3}=\pi\left(\sinh 2 r_{h}-2 r_{h}\right)
$$

and thus

$$
r_{e}=\left[\frac{3}{4}\left(\sinh 2 r_{h}-2 r_{h}\right)\right]^{1 / 3} .
$$

For $x<1, \sinh x<x+(1 / 3$ ! $) x^{3}+\left(2 / 5\right.$ !) $x^{5}$, so for $r_{h}<1 / 2$ we have

$$
\sinh 2 r_{h}-2 r_{h}<\frac{4}{3} r_{h}^{3}+\frac{8}{15} r_{h}^{5}
$$


Thus for $r_{h}<1 / 2$,

$$
r_{e}<\left[\frac{3}{4}\left(\frac{4}{3} r_{h}^{3}+\frac{8}{15} r_{h}^{5}\right)\right]^{1 / 3},
$$

and substituting this expression into (5.55) gives

$$
\begin{aligned}
\left|A_{h}^{\prime}(v)-A_{e}^{\prime}(v)\right| & <2 \operatorname{coth} r_{h}-\frac{2}{r_{h}}\left(1+\frac{2}{5} r_{h}^{2}\right)^{-1 / 3} \\
& <2 \operatorname{coth} r_{h}-\frac{2}{r_{h}}\left(1-\frac{2}{15} r_{h}^{2}\right)
\end{aligned}
$$

since $(1+x)^{-1 / 3}>1-(1 / 3) x$ for $x<1 / 2$. Since $\tanh x>x-(1 / 3) x^{3}$ for $x<1 / 2$, we have for $r_{h}<1 / 2$,

$$
\begin{aligned}
\left|A_{h}^{\prime}(v)-A_{e}^{\prime}(v)\right| & <\frac{2}{r_{h}-(1 / 3) r_{h}^{3}}-\frac{2}{r_{h}}\left(1-\frac{2}{15} r_{h}^{2}\right) \\
& <\frac{2}{r_{h}}\left(1+\frac{2}{3} r_{h}^{2}\right)-\frac{2}{r_{h}}\left(1-\frac{2}{15} r_{h}^{2}\right),
\end{aligned}
$$

since $(1-x)^{-1}<1+2 x$ for $x<1 / 2$. Thus for $r_{h}<1 / 2$,

$$
\left|A_{h}^{\prime}(v)-A_{e}^{\prime}(v)\right|<\frac{8}{5} r_{h} .
$$

LEMMA 5.10. Let $A_{e}(v, v)$ and $A_{h}(v, v)$ be the surface area of standard double bubbles enclosing two equal volumes $v$ in $\mathbb{R}^{3}$ and $\mathbb{\boxplus}^{3}$, respectively. Let $r_{e}$ and $r_{h}$ be the radii of these bubbles in $\mathbb{R}^{3}$ and $\mathbb{\boxplus}^{3}$, respectively. Then for $r_{h}<1 / 2$,

$$
\left|A_{h}^{\prime}(v, v)-A_{e}^{\prime}(v, v)\right|<3.8 r_{h} .
$$

Proof. From Remark 3.7, we have

$$
A_{h}^{\prime}(v, v)-A_{e}^{\prime}(v, v)=4 \operatorname{coth} r_{h}-\frac{4}{r_{e}}
$$

Since the volume of a standard equal-volume double bubble increases more rapidly with radius in $\mathbb{t}^{3}$ than in $\mathbb{R}^{3}, r_{h}>r_{e}$. Since coth $x$ decreases in $x$ for $x>0, A_{h}^{\prime}(v, v)-$ $A_{e}^{\prime}(v, v)$ is obviously positive for all $v$. It remains to find an upper bound on this quantity for small $v$.

From Proposition 3.5 and Remark 3.6, we have

$$
\begin{aligned}
v= & \frac{9}{8} \pi r_{e}^{3}=\frac{\pi}{2}\left(\sinh 2 r_{h}-2 r_{h}\right)\left(1+\frac{\sqrt{2} \cosh r_{h}}{\sqrt{7+\cosh 2 r_{h}}}\right) \\
& +\pi\left(\frac{\sqrt{2} r_{h} \cosh r_{h}}{\sqrt{7+\cosh 2 r_{h}}}-\tanh ^{-1}\left(\frac{\sqrt{2} \sinh r_{h}}{\sqrt{7+\cosh 2 r_{h}}}\right)\right),
\end{aligned}
$$

so

$$
\begin{aligned}
r_{e}=[ & \frac{4}{9}\left(\sinh 2 r_{h}-2 r_{h}\right)\left(1+\frac{\sqrt{2} \cosh r_{h}}{\sqrt{7+\cosh 2 r_{h}}}\right) \\
& \left.+\frac{8}{9}\left(\frac{\sqrt{2} r_{h} \cosh r_{h}}{\sqrt{7+\cosh 2 r_{h}}}-\tanh ^{-1}\left(\frac{\sqrt{2} \sinh r_{h}}{\sqrt{7+\cosh 2 r_{h}}}\right)\right)\right]^{1 / 3} .
\end{aligned}
$$


We use power series expansions to find upper bounds on each term of this formula. We begin with the second half of the formula. For $x<1, \tanh ^{-1} x>x+(1 / 3) x^{3}$, so for $r_{h}<1$ we have

$$
\begin{aligned}
r_{e}< & \frac{4}{9}\left(\sinh 2 r_{h}-2 r_{h}\right)\left(1+\frac{\sqrt{2} \cosh r_{h}}{\sqrt{7+\cosh 2 r_{h}}}\right) \\
& \left.+\frac{8}{9}\left(\frac{\sqrt{2}}{\sqrt{7+\cosh 2 r_{h}}}\left(r_{h} \cosh r_{h}-\sinh r_{h}\right)-\frac{1}{3}\left(\frac{\sqrt{2} \sinh r_{h}}{\sqrt{7+\cosh 2 r_{h}}}\right)^{3}\right)\right]^{1 / 3} .
\end{aligned}
$$

For $x<1 / 2, \cosh 2 x<1+4 x^{2}$ and $(1+x)^{-1 / 2}>1-(1 / 2) x$, so

$$
\frac{1}{\sqrt{7+\cosh 2 r_{h}}}>\frac{1}{\sqrt{8}}\left(1+\frac{1}{2} r_{h}^{2}\right)^{-1 / 2}>\frac{1}{\sqrt{8}}\left(1-\frac{1}{4} r_{h}^{2}\right) .
$$

In addition, $\sin x>x$ for $x>0$, so we have that for $r_{h}<1$,

$$
\begin{aligned}
\frac{1}{3}\left(\frac{\sqrt{2} \sinh r_{h}}{\sqrt{7+\cosh 2 r_{h}}}\right)^{3} & >\frac{1}{3}\left(\frac{r_{h}}{2}\left(1-\frac{1}{4} r_{h}^{2}\right)\right)^{3} \\
& =\frac{r_{h}^{3}}{24}\left(1-\frac{3}{4} r_{h}^{2}+\frac{3}{16} r_{h}^{4}-\frac{1}{64} r_{h}^{6}\right) \\
& >\frac{1}{24} r_{h}^{3}-\frac{1}{32} r_{h}^{5},
\end{aligned}
$$

since $(3 / 16) x^{4}>(1 / 64) x^{6}$ for $x<1$.

To deal with the term $\left(\sqrt{2} / \sqrt{7+\cosh 2 r_{h}}\right)\left(r_{h} \cosh r_{h}-\sinh r_{h}\right)$, we note that for all $r_{h}$,

$$
\frac{\sqrt{2}}{\sqrt{7+\cosh 2 r_{h}}} \leq \frac{1}{2}
$$

and for $r_{h}<1$,

$$
r_{h} \cosh r_{h}-\sinh r_{h}<\frac{1}{3} r_{h}^{3}+\frac{1}{15} r_{h}^{5}
$$

Substituting these approximations into (5.66) gives

$$
\begin{aligned}
r_{e}< & {\left[\frac{4}{9}\left(\sinh 2 r_{h}-2 r_{h}\right)\left(1+\frac{1}{2} \cosh r_{h}\right)\right.} \\
& \left.+\frac{8}{9}\left(\frac{1}{2}\left(\frac{1}{3} r_{h}^{3}+\frac{1}{15} r_{h}^{5}\right)-\left(\frac{1}{24} r_{h}^{3}-\frac{1}{32} r_{h}^{5}\right)\right)\right]^{1 / 3} .
\end{aligned}
$$


We now attack the first half of the expression. By the Taylor series expansions for $\sinh x$ and $\cosh x$ we have, for $r_{h}<1$,

$$
\sinh 2 r_{h}-2 r_{h}<\frac{4}{3} r_{h}^{3}+\frac{8}{15} r_{h}^{5}, \quad \cos r_{h}<1+r_{h}^{2} .
$$

We now have a polynomial approximation for $r_{e}$ that holds whenever $r_{h}<1 / 2$

$$
\begin{aligned}
r_{e}< & {\left[\frac{4}{9}\left(\frac{4}{3} r_{h}^{3}+\frac{8}{15} r_{h}^{5}\right)\left(1+\frac{1}{2}\left(1+r_{h}^{2}\right)\right)\right.} \\
& \left.+\frac{8}{9}\left(\frac{1}{2}\left(\frac{1}{3} r_{h}^{3}+\frac{1}{15} r_{h}^{5}\right)-\left(\frac{1}{24} r_{h}^{3}-\frac{1}{32} r_{h}^{5}\right)\right)\right]^{1 / 3} \\
= & \left(r_{h}^{3}+\frac{383}{540} r_{h}^{5}+\frac{16}{135} r_{h}^{7}\right)^{1 / 3} \\
< & \left(r_{h}^{3}+\frac{5}{6} r_{h}^{5}\right)^{1 / 3} .
\end{aligned}
$$

Using this approximation for $r_{e}$, we have, for $r_{h}<1 / 2$,

$$
\begin{aligned}
\left|A_{h}^{\prime}(v, v)-A_{e}^{\prime}(v, v)\right| & <4 \operatorname{coth} r_{h}-\frac{4}{r_{h}}\left(1+\frac{5}{6} r_{h}^{2}\right)^{-1 / 3} \\
& <4 \operatorname{coth} r_{h}-\frac{4}{r_{h}}\left(1-\frac{5}{18} r_{h}^{2}\right)
\end{aligned}
$$

since $(1+x)^{-1 / 3}>1-(1 / 3) x$ for $x<1 / 2$. Since $\tanh x>x-(1 / 3) x^{3}$ for $x<1$, we have, for $r_{h}<1 / 2$,

$$
\begin{aligned}
\left|A_{h}^{\prime}(v, v)-A_{e}^{\prime}(v, v)\right| & <\frac{4}{r_{h}-(1 / 3) r_{h}^{3}}-\frac{4}{r_{h}}\left(1-\frac{5}{18} r_{h}^{2}\right) \\
& <\frac{4}{r_{h}}\left(1+\frac{2}{3} r_{h}^{2}\right)-\frac{4}{r_{h}}\left(1-\frac{5}{18} r_{h}^{2}\right),
\end{aligned}
$$

since $(1-x)^{-1}<1+2 x$ for $x<1 / 2$. Thus for $r_{h}<1 / 2$,

$$
\left|A_{h}^{\prime}(v, v)-A_{e}^{\prime}(v, v)\right|<\frac{34}{9} r_{h}<3.8 r_{h}
$$

Proposition 5.11. Let $F_{h}(v)$ be defined as in (5.53). Then for $0<v \leq 0.002$, $F_{h}(v)>0$.

Proof. The proof is essentially the same as that of Proposition 5.5. By (5.53) and Lemmas 5.9 and 5.10, we have

$$
\left|F_{h}^{\prime}(v)-F_{e}^{\prime}(v)\right|<14 r_{h}(2 v)
$$

when $r_{h}(2 v)<1 / 2$. Direct computation from the volume formula (Remark 3.2) shows that the volume of a sphere in $\mathbb{t}^{3}$ of radius 0.1 is greater than 0.004 , so if $v \leq 0.002$, then $r_{h}(2 v)<0.1$ and $\left|F_{h}^{\prime}(v)-F_{e}^{\prime}(v)\right|<1.4$. As shown in the proof of Proposition 5.5, $F_{e}^{\prime}(v)>1.72$ for $v \leq 0.002$, so we conclude that $F_{h}^{\prime}(v)>0$ for $v \leq 0.002$. Since $F_{h}(0)$ is obviously zero, $F_{h}(v)$ must be positive when $0<v \leq 0.002$. 
We will now show that $F_{h}(v)$ is positive for intermediate volumes. We begin by finding a lower bound on the derivative, $F_{h}^{\prime}(v)$.

LEMMA 5.12. Let $F_{h}(v)$ be defined as in (5.53). Then for $v>0$,

$$
F_{h}^{\prime}(v)>-16 \frac{\pi}{v}
$$

Proof. From (5.53), we have

$$
F_{h}^{\prime}(v)=A_{h}^{\prime}\left(\frac{v}{2}\right)+A_{h}^{\prime}(v)+2 A_{h}^{\prime}(2 v)-2 A_{h}^{\prime}(v, v) .
$$

By Remark 3.3, $A^{\prime}(v)=2 \operatorname{coth} r_{h}(v)$, where $r_{h}(v)$ is the radius of a sphere of volume $v$. By Remark 3.7, $A_{h}^{\prime}(v, v)=4 \operatorname{coth} r_{h}(v, v)$, where $r_{h}(v, v)$ is the radius of one half of the double bubble enclosing two equal volumes $v$. We thus have

$$
F_{h}^{\prime}(v)=2 \operatorname{coth} r_{h}\left(\frac{v}{2}\right)+2 \operatorname{coth} r_{h}(v)+4 \operatorname{coth} r_{h}(2 v)-8 \operatorname{coth} r_{h}(v, v)
$$

To obtain a lower bound on $F_{h}^{\prime}(v)$, we first note that $\operatorname{coth} x>1$ for all $x>0$, and thus the sum of the first three terms is always greater than 8 . To work with the final term, we note that the volume of one half of a double bubble of radius $r_{h}$ is less than that of a sphere of the same radius. Thus for any $v, r_{h}(v, v)>r(v)$. We also note that $V\left(r_{h}\right)=\pi\left(\sinh 2 r_{h}-2 r_{h}\right)<\pi \sinh 2 r_{h}$, and thus $r_{h}(v)>(1 / 2) \sinh ^{-1}(v / \pi)$. By concavity (Proposition 4.3), $A_{h}^{\prime}(v, v)$ is decreasing in $v$ and thus in $r_{h}$ as well. By bounding $r_{h}(v, v)$ from below, we have bounded $A_{h}^{\prime}(v, v)$ from above, giving us a lower bound for the last term of $F_{h}^{\prime}(v)$. We conclude that

$$
F_{h}^{\prime}(v)>8-8 \operatorname{coth}\left(\frac{1}{2} \sinh ^{-1} \frac{v}{\pi}\right) .
$$

Using hyperbolic trigonometric identities, this simplifies to

$$
F_{h}^{\prime}(v)>8\left(1-\sqrt{1+\frac{\pi^{2}}{v^{2}}}-\frac{\pi}{v}\right)>-16 \frac{\pi}{v} .
$$

It is clear that this lower bound is increasing in $v$.

We will also need the following algebraic lemma.

LEMMA 5.13. Let $V_{h}(r, r)$ be the volume of one half of an equal-volume double bubble of radius $r$ in $\mathbb{M}^{3}$, and let $V_{h}^{\prime \prime}(r, r)$ be the second derivative of $V_{h}$ with respect to $r$. Then for all positive $r, V_{h}^{\prime \prime}(r, r)>0$.

Proof. Differentiating the formula in Proposition 3.5 twice gives

$$
\begin{aligned}
V_{h}^{\prime \prime}(r, r)= & 24 \pi \frac{\sqrt{2} \sinh ^{3} r}{(7+\cosh 2 r)^{3 / 2}}+2 \pi \sinh 2 r\left(1+\frac{\sqrt{2} \cosh r}{(7+\cosh 2 r)^{1 / 2}}\right) \\
& +3 \sqrt{2} \pi \frac{24 \sinh r \cosh ^{2} r-8 \cosh ^{4} r \sinh r+16 \sinh r+4 \sinh ^{3} r}{(7+\cosh 2 r)^{5 / 2}}
\end{aligned}
$$


All terms in this expression but one are positive for positive $r$; it therefore suffices to show that

$$
\frac{24 \sqrt{2} \pi \cosh ^{4} r \sinh r}{(7+\cosh 2 r)^{5 / 2}} \leq 2 \pi \sinh 2 r
$$

But $\cosh 2 r>2 \cosh ^{2} r$, and thus

$$
\frac{24 \sqrt{2} \pi \cosh ^{4} r \sinh r}{(7+\cosh 2 r)^{5 / 2}}<\frac{6 \sqrt{2} \pi \sinh r}{\sqrt{7+\cosh 2 r}}<\sqrt{2} \pi \sinh r<2 \pi \sinh 2 r .
$$

We use Lemmas 5.12 and 5.13 and a computer to show that $F_{h}$ is positive for all volumes between 0.002 and 100,000.

Proposition 5.14. Let $F_{h}(v)$ be defined as in (5.53). Then for $v \in\left[0.002,10^{5}\right]$, $F_{h}(v)>0$.

Proof. Our method is the same as that used to prove Proposition 5.8: we bound the derivative of $F_{h}^{\prime}(v)$ and use the computer to determine intervals for which $F_{h}(v)$ is positive. The only difference is that the error in the computer's calculations will be different; we need to calculate an upper bound on this error using our hyperbolic formulae. As in the spherical case, Mathematica uses Newton's method to calculate the radii of various spheres and double bubbles. Since the volume functions for spheres and double bubbles have positive first and second derivatives for positive $r$ (see Remark 3.2 and Lemma 5.13), if Newton's method takes a positive starting point and returns a radius such that the corresponding volume is within $\epsilon$ of the desired volume, then the radius is in fact near the desired radius. Since Mathematica's internal calculations have 16 digits of precision and no radius is ever greater than 10, we need only consider how much the error from Newton's method is magnified when these values are used to calculate areas.

Recall that the statement that Mathematica calculates the solution $x=x_{0}$ to the equation $f(x)=y_{0}$ with $n$ digits of accuracy means that

$$
\left|f\left(x_{0}\right)-y_{0}\right|<10^{-n}
$$

Let $\epsilon=10^{-n}$ (where for our purposes $n$ is 10) and let $\Delta>0$ be a lower bound on $\left|f^{\prime}(x)\right|$ over all $x$ in some interval $[a, b]$ that contains the exact solution $\tilde{x}$ and the calculated solution $x_{0}$ :

$$
\Delta \leq \min _{x \in[a, b]}\left|f^{\prime}(x)\right|
$$

Then we have

$$
\left|\tilde{x}-x_{0}\right|<\Delta^{-1} \epsilon .
$$


To find the error in the calculated value of $F_{h}(v)=2 A_{h}(v / 2)+A_{h}(v)+A_{h}(2 v)-$ $2 A_{h}(v, v)$, we will consider each term separately. We first consider the terms that are areas of spheres. In this case, the function $f$ in which we are interested is $V_{h}(r)$, the volume of a sphere of radius $r$. This function has increasing derivative (see Remark 3.2 ), so we may take $\Delta$ to be any number smaller than the value of the derivative at the smallest $r$ we are considering, which is $r(v / 2)$ for $v=0.002$. The value $r=0.062$ gives $V_{h}(r)<0.001-\epsilon$ and the derivative $V^{\prime}(r)>0.048$, so we may bound $V_{h}^{\prime}(r)$ by $\Delta_{1}=0.048$.

If we want to calculate the difference between $A_{h}(r)$, the area of a sphere of radius $r=r(v)$, and $A_{h}\left(r_{0}\right)$, where $r_{0}=r_{0}(v)$ is our calculated value for the radius $r$, then we have

$$
\left|A_{h}(r)-A_{h}\left(r_{0}\right)\right| \leq \Delta_{1}^{-1} \epsilon\left(\sup _{r \in\left(r_{0}-\Delta_{1}^{-1} \epsilon, r_{0}+\Delta_{1}^{-1} \epsilon\right)}\left|A_{h}^{\prime}(r)\right|\right)
$$

since $A_{h}^{\prime}(r)=4 \pi \sinh 2 r$ is increasing in $r$, we have, for sufficiently small $\Delta_{1}$,

$$
\begin{aligned}
\left|A_{h}(r)-A_{h}\left(r_{0}\right)\right| & \leq \Delta_{1}^{-1} \epsilon A_{h}^{\prime}\left(r_{0}+\Delta_{1}^{-1} \epsilon\right) \\
& \leq 4 \pi \Delta_{1}^{-1} \epsilon \sinh \left(2 r_{0}+2 \Delta_{1}^{-1} \epsilon\right) \\
& \leq 2 \pi \Delta_{1}^{-1} \epsilon e^{2 r_{0}+2 \Delta_{1}^{-1} \epsilon} \\
& <2 \pi \Delta_{1}^{-1} \epsilon e^{2 r_{0}}\left(1+4 \Delta_{1}^{-1} \epsilon\right),
\end{aligned}
$$

with the last inequality following because $e^{x}<1+2 x$ for $x<1$, and $2 \Delta_{1}^{-1} \epsilon<1$. For $\Delta_{1}=0.048$ we thus have

$$
\left|A_{h}(r)-A_{h}\left(r_{0}\right)\right|<42 \pi \epsilon e^{2 r_{0}} .
$$

To bound the error on the term of $F_{h}(v)$ that is the area of the double bubble, we first note that by Lemma 5.13, the derivative of $V_{h}(r, r)$ (the volume of one half of a double bubble of radius $r$ ) is increasing, and therefore we may take $\Delta$ to be any number smaller than the value of the derivative at the smallest $r$ we are considering. The value $r=0.082$ gives $V_{h}(r, r)<0.002-\epsilon$ and $V_{h}^{\prime}(r, r)>0.071$, so we may bound the derivative $V_{h}^{\prime}(r, r)$ by $\Delta_{2}=0.071$.

We now take the derivative of the area formula in Proposition 3.4, with $r=r(v, v)$ now referring to the radius of one component of a double bubble enclosing two equal volumes $v$, and $r_{0}=r_{0}(v, v)$ to our calculation of this value using Newton's method

$$
A_{h}^{\prime}(r, r)=4 \pi \sinh 2 r+4 \pi \sinh ^{2} r \frac{d \xi}{d r}+4 \pi \sinh 2 r \xi+4 \pi \frac{d \xi}{d r}
$$


where $\xi=\sqrt{2} \cosh r / \sqrt{7+\cosh 2 r}$. We thus have

$$
\begin{aligned}
A_{h}^{\prime}(r, r)= & 4 \pi \sinh 2 r+4 \pi \cosh ^{2} r\left(\frac{6 \sqrt{2} \sinh 2 r}{(7+\cosh 2 r)^{3 / 2}}\right) \\
& +4 \pi \sinh 2 r\left(\frac{\sqrt{2} \cosh r}{\sqrt{7+\cosh 2 r}}\right) \\
& <4 \pi \cosh ^{2} r(\sqrt{12})+4 \pi \sinh 2 r(1+\sqrt{2}) \\
& <4 \pi\left(\sqrt{12} e^{2 r}+\frac{1+\sqrt{2}}{2} e^{2 r}\right) \\
& <19 \pi e^{2 r}
\end{aligned}
$$

since $\cosh x<e^{x}$ and $\sinh x<e^{x} / 2$ when $x>0$. Thus we conclude that

$$
\begin{aligned}
\left|A_{h}(r, r)-A_{h}\left(r_{0}, r_{0}\right)\right| & <\Delta_{2}^{-1} \epsilon\left(19 \pi e^{2 r_{0}+2 \Delta_{2}^{-1} \epsilon}\right) \\
& <19 \pi \Delta_{2}^{-1} \epsilon e^{2 r_{0}}\left(1+4 \Delta_{2}^{-1} \epsilon\right) \\
& <268 \pi \epsilon e^{2 r_{0}}
\end{aligned}
$$

by the same chain of reasoning as in the case of $A_{h}(r)$ above.

We add up the errors from the four terms of $F_{h}(v)$ to get the final result:

$$
\begin{aligned}
\left|F_{h}(r)-F_{h}\left(r_{0}\right)\right|< & 84 \pi \epsilon e^{2 r_{0}(v / 2)}+42 \pi \epsilon e^{2 r_{0}(v)} \\
& +42 \pi \epsilon e^{2 r_{0}(2 v)}+268 \pi \epsilon e^{2 r_{0}(v, v) .}
\end{aligned}
$$

Let $E_{h}(v)$ be the right-hand side of this inequality.

We conclude that if Mathematica returns a positive value for $F_{h}\left(v_{0}\right)$, then, as in Proposition 5.8, $F_{h}(v)$ is guaranteed to be positive on the interval $\left[v_{0}, v_{0}-\right.$ $E_{h}(v) / G_{h}\left(v_{0}\right)$ ], where here $G_{h}(v)$ is the right-hand side of (5.79).

We write a simple Mathematica program to carry out this procedure; for the program's code, see Appendix B. We enter 0.002 and $10^{5}$ for the starting and ending values of $v$, and the program tells us that $F_{h}(v)$ is positive everywhere in between.

We now will use asymptotic approximations to show that $F_{h}$ is positive for large volumes. We begin by approximating the area of a sphere of volume $v$.

LEMMA 5.15. Let $A_{h}(v)$ be the area of a sphere of volume $v$ in $\mathbb{M}^{3}$. Then for all $v \geq 0$,

$$
A_{h}(v)>2 v-2 \pi+2 \pi \ln \left(\frac{2 v}{\pi}\right) .
$$

PROoF. From area and volume formulae (Remarks 3.1 and 3.2), we have

$$
\begin{aligned}
A_{h}(v) & =2 v-2 \pi+2 \pi e^{-2 r(v)}+4 \pi r(v) \\
& >2 v-2 \pi+4 \pi r(v),
\end{aligned}
$$


where $r(v)$ is the radius of a sphere of volume $v$. In addition, from the volume formula, $2 v / \pi=e^{2 r}-e^{-2 r}-4 r<e^{2 r}$, so $r(v)>(1 / 2) \ln (2 v / \pi)$. Thus $A_{h}(v)>$ $2 v-2 \pi+2 \pi \ln (2 v / \pi)$.

It is easy to see that the right-hand side of (5.97) becomes asymptotically close to $A_{h}(v)$ as $v$ gets large. To derive a similar approximation for $A_{h}(v, v)$ for double bubbles, we will need two algebraic lemmas.

LEMMA 5.16. Let $\xi=\sqrt{2} \cosh r / \sqrt{7+\cosh 2 r}$. Then for $r>1.5$,

$$
\tanh ^{-1}(\xi \tanh r)<r-\ln 2+9 e^{-2 r}
$$

Proof. Note first that

$$
\xi \tanh r=\frac{\sqrt{2}\left(\left(e^{r}-e^{-r}\right) / 2\right)}{\sqrt{7+\left(\left(e^{2 r}+e^{-2 r}\right) / 2\right)}}
$$

Since $\tanh ^{-1} x=(1 / 2) \ln ((1+x) /(1-x))$, we have, for $r>3 / 2$

$$
\begin{aligned}
\tanh ^{-1}(\xi \tanh r) & =\frac{1}{2} \ln \left(\frac{\sqrt{14+e^{2 r}+e^{-2 r}}+e^{r}-e^{-r}}{\sqrt{14+e^{2 r}+e^{-2 r}}-e^{r}+e^{-r}}\right) \\
& =\frac{1}{2} \ln \left(\frac{\sqrt{1+\left(14 e^{-2 r}+e^{-4 r}\right)}+1-e^{-2 r}}{\sqrt{1+\left(14 e^{-2 r}+e^{-4 r}\right)}-1+e^{-2 r}}\right) .
\end{aligned}
$$

Since $1+(1 / 2) x-(1 / 4) x^{2}<\sqrt{1+x}<1+(1 / 2) x$ for $x<1$, and $14 e^{-2 r}+e^{-4 r}<1$ for $r>3 / 2$,

$$
\tanh ^{-1}(\xi \tanh r)<\frac{1}{2} \ln \left(\frac{1+(1 / 2)\left(14 e^{-2 r}+e^{-4 r}\right)+1-e^{-2 r}}{1+(1 / 2)\left(14 e^{-2 r}+e^{-4 r}\right)-(1 / 4)\left(14 e^{-2 r}+e^{-4 r}\right)^{2}-1+e^{-2 r}}\right) .
$$

Since $e^{-4 r}<e^{-2 r}$ for $r>0$, we have for $r>3 / 2$,

$$
\begin{aligned}
\tanh ^{-1}(\xi \tanh r) & <\frac{1}{2} \ln \left(\frac{2+8 e^{-2 r}}{8 e^{-2 r}-56 e^{-4 r}}\right) \\
& =\frac{1}{2} \ln \left(\frac{2\left(1+4 e^{-2 r}\right)}{8 e^{-2 r}\left(1-7 e^{-2 r}\right)}\right) \\
& <\frac{1}{2} \ln \left(\frac{1}{4} e^{2 r}\left(1+4 e^{-2 r}\right)\left(1+14 e^{-2 r}\right)\right) \\
& =r-\ln 2+\frac{1}{2} \ln \left(1+4 e^{-2 r}\right)+\frac{1}{2} \ln \left(1+14 e^{-2 r}\right),
\end{aligned}
$$

since $1 /(1-x)<1+2 x$ for $x<1 / 2$ and $7 e^{-2 r}<1 / 2$ for $r>3 / 2$. Finally, since $\ln (1+$ $x)<x$ for all $x$, we have for $r<3 / 2$,

$$
\tanh ^{-1}(\xi \tanh r)<r-\ln 2+9 e^{-2 r}
$$


LEMMA 5.17. Let $V_{h}(r, r)$ be one volume enclosed by a standard double bubble in $\mathbb{M}^{3}$ consisting of two spherical caps of radius $r$ with a flat disc in between. If $r \geq 5$, then $V_{h}(r, r)>0.99 \pi \sinh 2 r$.

Proof. As above, let $\xi=\sqrt{2} \cosh r / \sqrt{7+\cosh 2 r}$. By Proposition 3.5, we have for $r>3 / 2$

$$
\begin{aligned}
V_{h}(r, r) & =\frac{\pi}{2}(\sinh 2 r-2 r)(1+\xi)+\pi\left(r \xi-\tanh ^{-1}(\xi \tanh r)\right) \\
& =\pi\left(\frac{1+\xi}{2}\right) \sinh 2 r-\pi r-\pi \tanh ^{-1}(\xi \tanh r) \\
& >\pi\left(\frac{1+\xi}{2}\right) \sinh 2 r-2 \pi r+\pi \ln 2-9 \pi e^{-2 r}
\end{aligned}
$$

by Lemma 5.16. Since $9 e^{-2 x}<\ln 2$ for $x>3 / 2$, we have

$$
V_{h}(r, r)>\pi\left(\frac{1+\xi}{2}\right) \sinh 2 r-2 \pi r
$$

Note that $\xi$ is increasing in $r$, since

$$
\frac{d \xi}{d r}=\frac{\sqrt{2} \sinh r(7+\cosh 2 r)-\sqrt{2} \cosh r \sinh 2 r}{(7+\cosh 2 r)^{3 / 2}}
$$

and the numerator is equal to $6 \sqrt{2} \sinh r$, which is positive for $r>0$. In addition, it is easy to see that $\xi<1$ for all $r$. Simple calculation shows that when $r=5,1-(1+\xi) / 2<$ 0.001 , and thus $|1-(1+\xi) / 2|<0.001$ whenever $r \geq 5$. In addition, $0.009 \pi \sinh 10>$ $10 \pi$ and $0.018 \pi \cosh 10>2 \pi$, so for $r \geq 5,0.009 \pi \sinh 2 r>2 \pi r$. Thus for $r \geq 5$,

$$
V(r, r)>\pi(1-0.001) \sinh 2 r-0.009 \pi \sinh 2 r=0.99 \pi \sinh 2 r .
$$

We use these two lemmas to approximate $A_{h}(v, v)$ for large volumes.

LEMMA 5.18. Let $A_{h}(v, v)$ be the area of the standard double bubble in $\mathbb{\boxplus}^{3}$ enclosing two equal volumes $v$. Then for $v \geq 10^{5}, A_{h}(v, v)<4 v-2 \pi+4 \pi \ln (v / \pi)+0.14$.

PROoF. From the area and volume formulae (Propositions 3.4 and 3.5), we have

$$
\begin{aligned}
A_{h}(v, v)= & 4 v+(1+\xi)\left(2 \pi e^{-2 r}-2 \pi+4 \pi r\right) \\
& +2 \pi(2 \xi-1)-4 \pi\left(r \xi-\tanh ^{-1}(\xi \tanh r)\right),
\end{aligned}
$$


where $r$ is the radius of the standard double bubble enclosing two regions of volume $v$, and $\xi$ is, as usual, $\sqrt{2} \cosh r / \sqrt{7+\cosh 2 r}$. Simplifying and using Lemmas 5.16 and 5.17 and the fact that $\xi<1$, we have for $r \geq 5$,

$$
\begin{aligned}
A_{h}(v, v) & <4 v-2 \pi+4 \pi r+4 \pi \tanh ^{-1}(\xi \tanh r)+4 \pi e^{-2 r} \\
& <4 v-2 \pi+8 \pi r-4 \pi \ln 2+40 \pi e^{-2 r} \\
& <4 v-2 \pi+4 \pi \sinh ^{-1}\left(\frac{v}{0.99 \pi}\right)-4 \pi \ln 2+40 \pi e^{-2 r}
\end{aligned}
$$

Since $\sinh ^{-1} x<\ln x+\ln 2+1 / 4 x^{2}$ for $x>1$, and $v / 0.99 \pi>1$ for $r \geq 5$, we have for $r \geq 5$,

$$
\begin{aligned}
A_{h}(v, v) & <4 v-2 \pi+4 \pi \ln \left(\frac{v}{\pi}\right)-4 \pi \ln 0.99+\frac{\pi(0.99 \pi)^{2}}{v^{2}}+40 \pi e^{-2 r} \\
& <4 v-2 \pi+4 \pi \ln \left(\frac{v}{\pi}\right)+0.127+10^{-6}+0.006 \\
& <4 v-2 \pi+4 \pi \ln \left(\frac{v}{\pi}\right)+0.14
\end{aligned}
$$

since $v>10^{4}$ when $r \geq 5$.

Proposition 5.19. Let $F_{h}(v)$ be defined as in (5.53). Then for $v \geq 10^{5}, F_{h}(v)>0$.

Proof. By (5.53), $F_{h}(v)=2 A_{h}(v / 2)+A_{h}(v)+A_{h}(2 v)-2 A_{h}(v, v)$. By Lemmas 5.15 and 5.18 , we have

$$
\begin{aligned}
F_{h}(v)> & 2\left(v-2 \pi+2 \pi \ln \left(\frac{v}{\pi}\right)\right)+\left(2 v-2 \pi+2 \pi \ln \left(\frac{2 v}{\pi}\right)\right) \\
& +\left(4 v-2 \pi+2 \pi \ln \left(\frac{4 v}{\pi}\right)\right)-2\left(4 v-2 \pi+4 \pi \ln \left(\frac{v}{\pi}\right)+0.14\right) \\
= & 6 \pi \ln 2-4 \pi-0.28
\end{aligned}
$$

which is greater than zero.

Note that by choosing a larger lower bound for volume, we can make arbitrarily small the constant which we have calculated as 0.14 . Thus the limit as $v$ approaches infinity of $F_{h}(v)$ is $6 \pi \ln 2-4 \pi \approx 0.499146$. This is the value that $F_{h}(v)$ appears to approach in Figure 5.2, so this plot is indeed an accurate picture of $F_{h}(v)$.

We now have all the tools necessary to prove that a double bubble enclosing two equal volumes in $\mathbb{\boxplus}^{3}$ must have two connected regions.

Proof of Proposition 5.2. By Proposition 4.8 and Lemma 4.10, the two regions of equal volume $v$ are connected if $F_{h}(v)=2 A_{h}(v / 2)+A_{h}(v)+A_{h}(2 v)-2 A_{h}(v, v)$ is greater than zero. By Propositions 5.11, 5.14, and 5.19, $F_{h}(v)$ is greater than zero for all $v$.

6. The structure of area-minimizing double bubbles. Our Theorems 6.5 and 6.10 extend the Hutchings structure theorem [10, Theorem 5.1] for minimizing double bubbles from $\mathbb{R}^{n}$ to $S^{n}$ and $\mathbb{q}^{n}$. Before we prove these theorems, we need a stronger regularity theorem (Proposition 6.1) using the fact that the bubble is a hypersurface 
of revolution about a line (see Proposition 2.4). We also need the fact that the bubble is connected, which we show in Lemma 6.2, and that certain pieces of bubbles must be spherical, which we show in Lemma 6.3. We generalize a result of Foisy [5, Theorem 3.6] to show that a minimizing double in $\mathbb{M}^{n}$ must touch its axis of symmetry (Proposition 6.8), so the same structure theorem applies in $\mathbb{M}^{n}$ as in $\mathbb{R}^{n}$ (Theorem 6.10). In $S^{n}$, we do not have such a result, and thus the situation is more complicated. In our structure theorem for $S^{n}$ (Theorem 6.5), we consider only cases when we know that one region is connected, and classify double bubbles based on whether this region intersects part, all, or none of the axis of symmetry.

The proofs in this section assume $n \geq 3$. The statements are true for $S^{2}$ by [12] and for $\mathbb{\boxplus}^{2}$ by Theorem 2.7.

In this section, all figures of $S^{2}$ are drawn using the stereographic projection of the upper hemisphere, and all figures of $\llbracket^{2}$ are drawn using the Poincaré disc model.

Proposition 6.1. An area minimizing double bubble $B$ in $S^{n}$ or $\mathbb{\boxplus}^{n}$ consists of finitely many smooth constant mean curvature hypersurfaces of revolution meeting in threes at 120-degree angles and meeting the axis of symmetry orthogonally.

Proof. By Proposition 2.4, $B$ is a hypersurface of revolution about a geodesic line. Like Hutchings [10, Section 5], we consider the half-planar generating curves with a smoothly varying density function dependent on the distance from the axis of symmetry. These curves must be smooth and meet in threes at 120-degree angles. The only potential problem is at the axis, where the density goes to zero, but Proposition 4.11 rules out infinite complexity here. A standard variational argument shows that the hypersurface must have constant mean curvature and intersect the axis orthogonally.

LEMMA 6.2. In $\mathbb{R}^{n}, S^{n}$, or $\mathbb{U}^{n}$, an area-minimizing double bubble for two given volumes is connected (and thus its generating curve is as well).

PROoF. If not, we can translate one component of the bubble until it touches another, creating an illegal singularity.

NotATION. Let $\Sigma$ (in $\mathbb{R}^{n}, S^{n}$, or $\mathbb{\sharp}^{n}$ ) be an area-minimizing double bubble of revolution about an axis $L$, with a generating curve $\Gamma$ in the upper half plane (of $\mathbb{R}^{2}, S^{2}$, or $\left.\mathbb{U}^{2}\right)$ that consists of $\operatorname{arcs} \bar{\Gamma}_{i}$, with interiors $\Gamma_{i}$ ending either at the axis or in threes at vertices $v_{i j k}$.

LEMMA 6.3. In $\mathbb{R}^{n}, S^{n}$, or $\mathbb{Q}^{n}$, if removing one point in the interior of one of the $\Gamma_{i}$ disconnects $\Gamma$, then $\Gamma_{i}$ is a constant curvature arc which, if completed, would hit the axis of rotation $L$ orthogonally. In particular, in $\mathbb{R}^{n} \Gamma_{i}$ is a piece of a straight line or circle, in $S^{n}$ it is a piece of a circle, and in $\mathbb{\boxplus}^{n}$ it is a piece of a circle, horocycle, hypocircle, or geodesic line.

Proof. We consider a separating set consisting of one of the points in the interior of $\Gamma_{i}$ and apply [11, Proposition 5.2] for $\mathbb{R}^{n}$, Theorem 7.2 for $S^{n}$, or Theorem 7.6 for

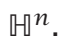


COROLlaRY 6.4. Any $\Gamma_{i}$ which intersects the axis of rotation L must be a constant curvature arc and intersect $L$ orthogonally.

\subsection{Structure of minimizers in $S^{n}$}

THEOREM 6.5. Consider a nonstandard area-minimizing double bubble in $S^{n}$ in which at least one of the three regions is connected, and label one connected region the exterior. Then the bubble is one of the following.

(1) If the exterior intersects part, but not all, of the axis of rotation, then the bubble consists of a topological sphere with a tree of toroidal bands attached. The two caps are pieces of spheres and the root of the tree has just one branch. (See Figure 6.1.)

(2) If the exterior intersects the entire axis of rotation, then the bubble consists entirely of toroidal bands and has the graph structure of a bipartite tree. (See Figure 6.2.)

(3) If the exterior does not intersect the axis of rotation, then the bubble has the graph structure of a bipartite graph with only one cycle. The regions corresponding to nodes in this cycle intersect the axis of rotation and are the only regions to do so. If the cycle is not trivial (i.e., one node), then each node represents a region which is a topological sphere. If the cycle is trivial, then that region is a topological torus containing the axis of rotation. The other nodes all represent toroidal bands. (See Figure 6.3.)

Proof. By Proposition 6.1, we know that the bubble is a hypersurface of revolution about a geodesic line and consists of constant mean curvature pieces which meet in threes at 120-degree angles and meet the axis of symmetry orthogonally. By Lemma 6.2 we know that the generating curve $\Gamma$ is connected. By Lemma 6.3, if removing a point from a $\Gamma_{i}$ disconnects $\Gamma$, then this $\Gamma_{i}$ is a piece of a circle centered on $L$. There are three cases to consider.

CASE 1 (Both the exterior and the bubble touch the axis). We follow the method of Hutchings [10, Section 5]. Consider the generating curve $B$ of the boundary of the exterior. This curve $B$ must touch $L$ twice. Start at one end and follow $B$ to the first triple point $p$. Let $C$ be the portion of the boundary between $R_{1}$ and $R_{2}$ (the two other regions) which meets $B$ at $p$. (See Figure 6.4.) Extend $C$ along the boundary of this component of $R_{1}$ until it touches the axis $L$ or comes back to $B$.

We consider first the subcase where $C$ comes to touch $L$ without returning to $B$. (See Figure 6.5.) We claim that this bubble must be the standard double bubble. If not, then either $B$ or $C$ has a branch off of it at some point. This cannot happen at the boundary between $R_{1}$ and $R_{2}$, for the exterior is connected. Nor can it happen at the boundary between the exterior and $R_{1}$, for this portion of $B$ was constructed to be the boundary of both of these regions. Thus there can only be a branch in the boundary between the exterior and $R_{2}$, which we denote $D$. Let $q$ be the point of branching closest to $p$. (See Figure 6.6.) Between $p$ and $q, D$ must be a piece of a circle centered on $L$ by Lemma 6.3, for removal of a point separates $\Gamma$. We can therefore roll the (toroidal) component of the bubble on one side of $q$ around the portion of a sphere generated by $D$ until it touches the component of the bubble on the other side of $p$, preserving 


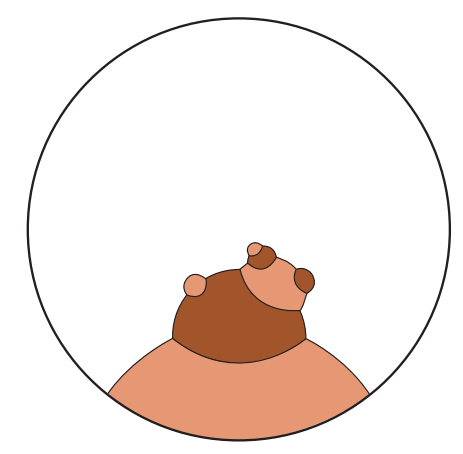

FIGURE 6.1. The generating curve for a nonstandard area-minimizing double bubble in which the exterior intersects part, but not all, of the axis of revolution; the bubble consists of a topological sphere with a tree of toroidal bands attached, and the root of the tree has one branch.

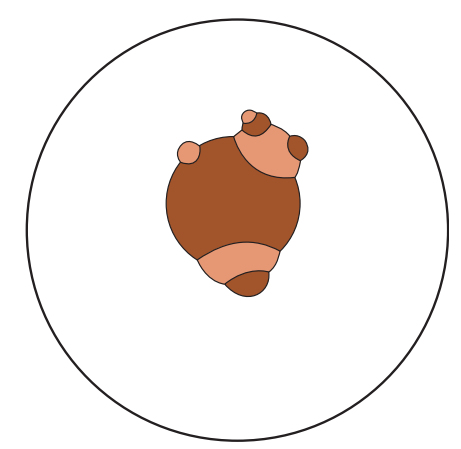

FIGURE 6.2. The generating curve for a nonstandard area-minimizing double bubble in which the exterior intersects the entire axis of revolution; the bubble consists entirely of toroidal bands.
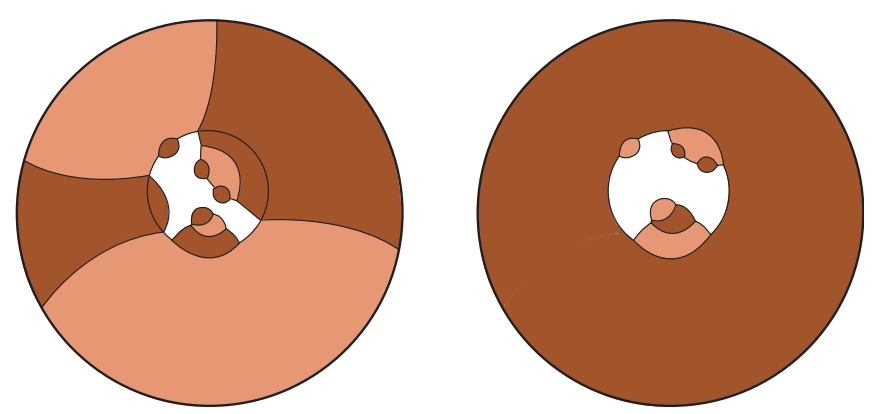

FIGURE 6.3. The generating curve for a nonstandard area-minimizing double bubble in which the exterior does not intersect the axis of revolution. The bubble's graph structure has at most one cycle, whose nodes correspond to regions intersecting the axis. If this cycle is nontrivial (left), then each region in the cycle is a topological sphere with trees of toroidal bands attached. If the cycle is trivial (right), then the region intersecting the axis is a topological torus with trees of toroidal bands attached. 


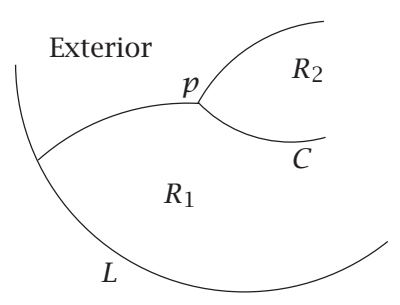

FIGURE 6.4. The generating curve first branches at point $p$.

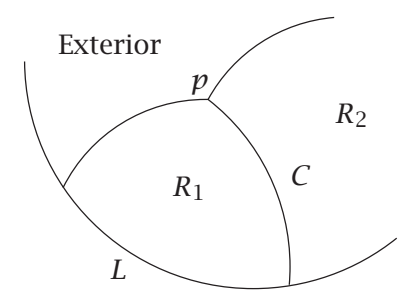

FiguRE 6.5. $C$ intersects the axis of rotation $L$ without returning to the boundary of the exterior.

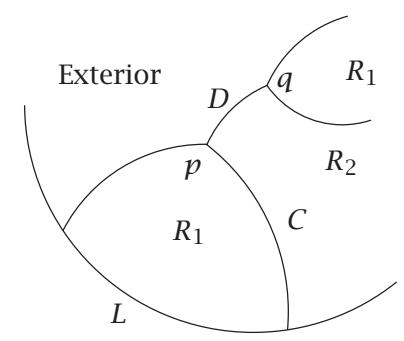

FIGURE 6.6. The boundary between the exterior and $R_{2}$ first branches at point $q$.

area and volume but creating an illegal singularity, since the $\Gamma_{i}$ would meet in fours, contradicting Proposition 6.1; see Figure 6.7 and [10, Section 5]. (Note that this rolling is an operation on the bubble as a whole and not the generating curve; indeed, the bubble does not remain rotationally symmetric about $L$ after this operation.) Thus there is no branch off of $D$ as well, and thus $\Gamma$ consists only of the three $\operatorname{arcs} B, C$, and $D$, and the bubble must be the standard bubble, for the arcs must be circular, hit the axis orthogonally and meet at 120-degree angles, and by Proposition 2.6 this bubble is unique.

We next consider the subcase where $C$ comes back to $B$ at a point $q$, enclosing a component of $R_{2}$. (See Figure 6.8.) There cannot be any branching on the boundary between the exterior and the component of $R_{1}$ in question after the point $q$, for then we could roll the point of branching to $q$, giving a contradiction as above. It follows that the exterior and this component of $R_{1}$ are the only components which touch the edge, and are topological spheres. The rest of the components must all be topological tori 


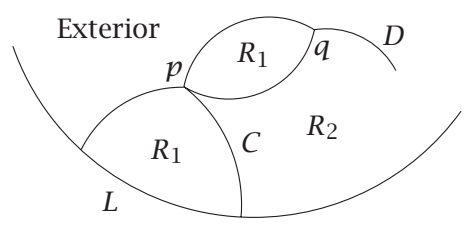

FIGURE 6.7. After rolling the toroidal component of $R_{1}$ along the spherical surface corresponding to $D$, we obtain an illegal singularity. (Note that double bubble does not remain a surface of revolution after rolling, and that this figure is not the generating curve after rolling, but rather is the hemisphere slice where contact happens.)

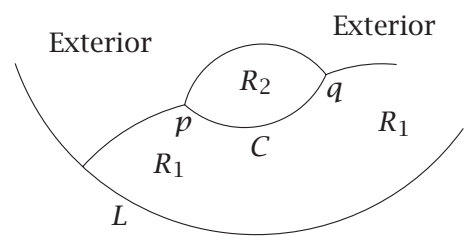

FIGURE 6.8. $C$ returns to the boundary of the exterior at the point $q$.

since they do not touch the axis. The bubble's graph structure must be a bipartite tree in which the root has one branch; the root corresponds to the component of region $R_{1}$ which touches the axis, and the branch to the boundary between this component of $R_{1}$ and the component of $R_{2}$ which it touches.

CASE 2 (The exterior touches the axis of rotation and the bubble does not). The resulting graph structure for the other two regions cannot have any cycles, for then the exterior would be disconnected. Thus the graph is a bipartite tree. Since only the exterior touches the axis, every node in this tree represents a toroidal band. (See Figure 6.2.)

CASE 3 (The exterior does not touch the axis). The generating curve must touch the axis an even number of times, for the other two regions $R_{1}$ and $R_{2}$ must alternate at the axis. Since the bubble is connected, it is possible to start at a point where the bubble touches the axis and follow a path to the boundary of the exterior. By the rolling argument, there can be no branching on this path. Thus the components of $R_{1}$ and $R_{2}$ which intersect the axis are either a single topological torus containing the axis or an even number of topological spheres. Between the exterior (which must be a single topological torus by connectedness) and the regions touching the axis there can be further topological tori belonging to regions $R_{1}$ and $R_{2}$, and we see that the graph structure must be a bipartite graph with one (possibly trivial) cycle, the cycle corresponding to those regions touching the axis.

COROLlARY 6.6. Any nonstandard area-minimizing double bubble in $S^{n}$ in which at least two of the three regions are connected is one of the following (see Figure 6.9). 


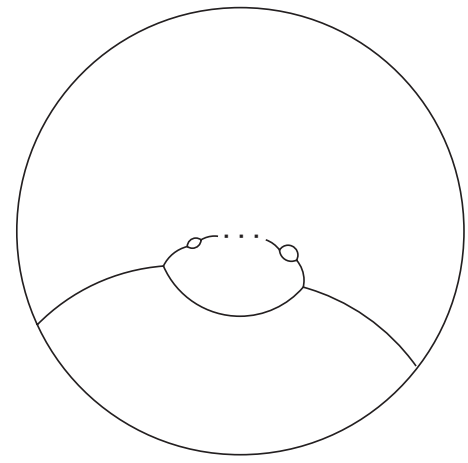

(a)

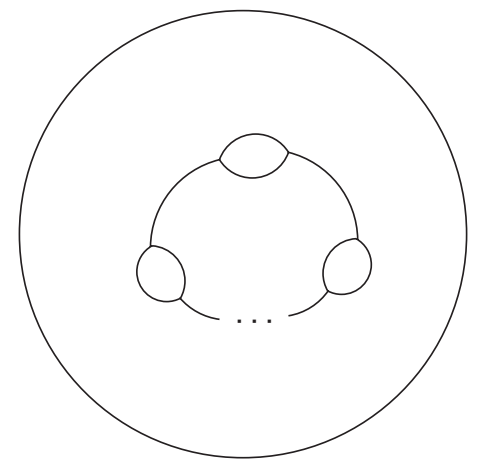

(b)

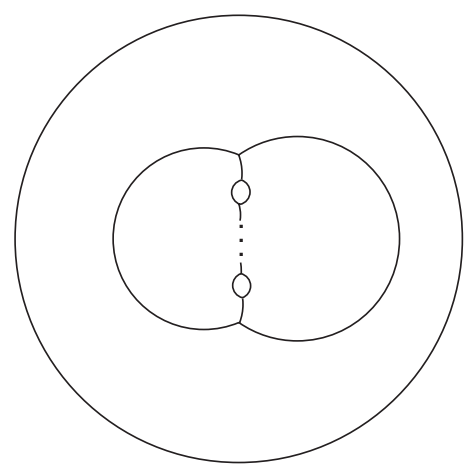

(c)

FIGURE 6.9. The three cases of Corollary 6.6.

(1) A topological sphere intersecting the axis of rotation, with one toroidal band around it and some number of toroidal bands around that. This is analogous to the $1+k$ bubble of Reichardt et al. [17].

(2) A region consisting of a single toroidal band, which has some number of toroidal bands on it belonging to the (possibly) disconnected region. In this case, the bubble does not intersect the axis of rotation.

(3) Two adjacent toroidal bands representing the two connected regions, with some number of toroidal bands inserted at their interface, which belong to the region which intersects the axis. In this case, the bubble does not intersect the axis of rotation.

Proof. This follows directly from consideration of the cases in Theorem 6.5.

COROLLARY 6.7. Any nonstandard area-minimizing double bubble in $S^{n}$ in which all three regions are connected is one of the following.

(1) A topological sphere intersecting the axis of rotation, with one toroidal band on top of it. (The third region is also a topological sphere intersecting the axis.)

(2) Two topological tori which touch and neither intersect nor contain the axis. (The third region is a topological torus which contains the axis of symmetry.) 


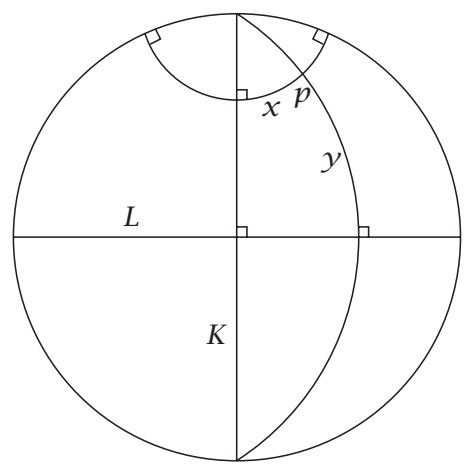

FIGURE 6.10. Construction of a coordinate system on $\mathbb{H}^{2}$.

Proof. This follows directly from consideration of the cases in Theorem 6.5.

\subsection{Structure of minimizers in $\mathbb{\boxplus}^{n}$}

Proposition 6.8. A minimizing double bubble in $\mathbb{a}^{n}$ intersects its axis of symmetry.

Proof. Like Foisy [5], we deform the bubble toward the axis while maintaining volume and decreasing surface area.

We work with the generating curve $\Gamma$ in the hyperbolic plane $\mathbb{\boxplus}^{2}$. Choose a geodesic line $K$ orthogonal to the axis of revolution $L$. We place a coordinate system on $\mathbb{t}^{2}$ as follows: let the coordinates of a point $p$ be $(x, y)$, where $x$ is the geodesic distance between the point and $K$, and $y$ is the distance between $p$ and $L$ measured along the hypocircle through $p$ that is a constant distance $x$ from $K$. (See Figure 6.10.) These coordinates divide the plane into four-sided figures whose top and bottom sides are geodesic segments and whose left and right sides are portions of hypocircles, with all pieces meeting at right angles. If the hypocircles are a distance $d x$ apart and the geodesics are a distance $d y$ apart, then the area of an infinitesimal grid "square" is $d x d y$. If the square is a distance $r$ from the axis of revolution $L$, then its volume when revolved is proportional to $\sinh ^{n-2} r d x d y$. If a portion of our generating curve intersects such a square for a length $d l$, then its surface area when revolved is proportional to $\sinh ^{n-2} r d l$.

Given a point $p=(x, y)$, we can find $y$, the distance between $p$ and $L$ along the appropriate hypocircle, as a function of $x$, the geodesic distance from $K$, and $r$, the geodesic distance between $p$ and $L$. Let this function be $y=G_{x}(r)$, and let the derivative $d y / d r$ be $g_{x}(r)$. We note that $g_{x}(r) \geq 1$ for all $x$ and $r$ and that $g_{x}(r)$ is continuously differentiable.

We seek a deformation of the generating curve which moves points $p=(x, y)$ along hypocircles toward the axis $L$ and preserves volume to first order. As we deform an infinitesimal square, $d x$ by $d y$, the distance $r$ from this square to $L$ will change and the differential length $d y$ will change, but the differential length $d x$ will remain constant. Thus to preserve volume to first order, we need only preserve $\sinh ^{n-2} r d y$. If we also ensure that differential length $d y$ always increases (to first order), such a transformation will also decrease surface area to first order, for the surface area 
associated with an infinitesimal piece is proportional to $\sinh ^{n-2} r \sqrt{d x^{2}+d y^{2}}$, which is equal to $\sinh ^{n-2} r d y \sqrt{1+d x^{2} / d y^{2}}$.

We construct a vector field with norm $c_{x}(r)$ pointing along the hypocircles towards $L$, and consider the flow $f_{x, \epsilon}(r)=r-\epsilon c_{x}(r)$, which is the distance from $L$ to which we will move a point $p=(x, y)$ which is initially a distance $r$ away from $L$. If we consider a differential length $d y$ along a certain hypocircle, and find the corresponding differential distance from $L, d r$, we have $d y=g_{x}(r) d r$ by above. Now, if we consider how $d r$ changes under the transformation $f$, we find that $\widehat{d r}=f_{x, \epsilon}^{\prime}(r) d r$ and thus $\widehat{d y}=g_{x}\left(f_{x, \epsilon}(r)\right) f_{x, \epsilon}^{\prime}(r)\left(d y / g_{x}(r)\right)$, where $\widehat{d r}$ and $\widehat{d y}$ represent $d r$ and $d y$ after the transformation.

We note that all we now need for volume to be preserved is for the equation

$$
\sinh ^{n-2}\left(f_{x, \epsilon}(r)\right) \widehat{d y}=\sinh ^{n-2} r d y
$$

to hold to first order in $\epsilon$. This is the condition that (to first order in $\epsilon$ )

$$
\left(\frac{\sinh ^{n-2}\left(r-\epsilon c_{x}(r)\right)}{\sinh ^{n-2} r}\right)\left(\frac{g\left(r-\epsilon C_{x}(r)\right)}{g(r)}\right)\left(1-\epsilon c_{x}^{\prime}(r)\right)=1 .
$$

Note that for any continuously differentiable function $h(x), h(x+\epsilon) / h(x)=1+$ $\epsilon\left(h^{\prime}(x) / h(x)\right)$ to first order in $\epsilon$. Thus the above condition reduces to

$$
\left(1-\epsilon c_{x}(r)(n-2) \operatorname{coth} r\right)\left(1-\epsilon c_{x}(r) \frac{g^{\prime}(r)}{g(r)}\right)\left(1-\epsilon c_{x}^{\prime}(r)\right)=1 .
$$

This gives the differential equation

$$
c_{x}(r)\left((n-2) \operatorname{coth} r+\frac{g^{\prime}(r)}{g(r)}\right)+c_{x}^{\prime}(r)=0
$$

which has the solution $c_{x}(r)=A\left(\operatorname{csch}^{n-2} r / g(r)\right)$, giving us a vector field which preserves volume. Note also that $c_{x}(r)$ is always positive (for positive $r$ ). Deforming toward the axis $L$ thus decreases $r$ everywhere, so for volume to be preserved $d y$ must increase everywhere, and thus, as desired, surface area decreases.

REMARK 6.9. This method generalizes to show that in $S^{n}$, any bubble whose generating curve is contained in any half of the hemisphere must touch the axis; one translates along concentric circles about a point on the axis. In $\mathbb{R}^{n}$, this method gives a deformation vector field of $1 / r^{n-2}$ downward along lines orthogonal to the axis of revolution. We note that this deformation agrees with Foisy's formula [5], $r \mapsto\left(r^{n-1}-\epsilon\right)^{1 /(n-1)}$, to first order in $\epsilon$.

THEOREM 6.10. Any nonstandard area-minimizing double bubble in $\mathbb{\boxplus}^{n}$ is a surface of revolution about some line, and consists of a topological sphere with a tree of toroidal bands attached. The two caps are pieces of spheres, and the root of the tree has just one branch. (See Figure 6.11.) 


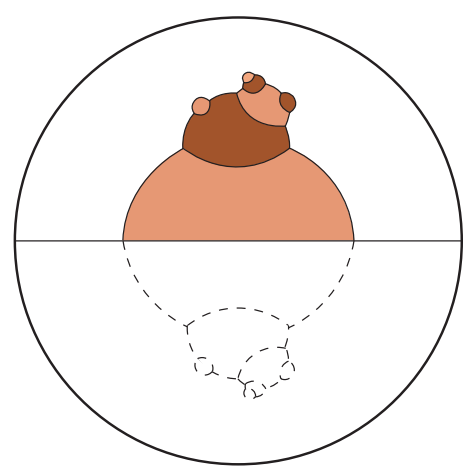

FIGURE 6.11. Generating curve for a possible nonstandard area-minimizing double bubble in $\mathbb{t}^{n}$.

Proof. The proof is identical to that of Hutchings [10, Theorem 5.1], making use of Propositions 6.1 and 6.8 and Lemmas 6.2 and 6.3.

COROLLARY 6.11. Any nonstandard area-minimizing double bubble in $\mathbb{\boxplus}^{n}$ in which at least one of the enclosed regions is connected consists of a topological sphere intersecting the axis of symmetry with one toroidal band around it, and some number of toroidal bands belonging to the first region around that. This is analogous to the $1+k$ bubble of Reichardt et al. [17].

Proof. This follows directly from consideration of the cases in Theorem 6.10.

COROLlARY 6.12. Any nonstandard area-minimizing double bubble in $\mathbb{\boxplus}^{n}$ in which both enclosed regions are connected is a surface of revolution about some line and consists of a topological sphere intersecting the axis of rotation with one toroidal band on top of it.

Proof. This follows directly from Theorem 6.10.

7. Stability of double bubbles. We now show how the fundamental instability argument of Hutchings et al. [11] generalizes to $S^{n}$ and $\mathbb{\boxplus}^{n}$ (Theorems 7.2 and 7.6) and then apply it to rule out nonstandard double bubbles in $S^{n}$ and $\mathbb{t}^{n}$ in which all regions are known to be connected (Propositions 7.3 and 7.7).

As in the previous section, all figures of $S^{2}$ are drawn using the stereographic projection of the upper hemisphere, and all figures of $\mathbb{\boxplus}^{2}$ are drawn using the Poincaré disc model.

Proposition 7.1 [11, Proposition 5.2]. Consider an area-minimizing double bubble in $\mathbb{R}^{n}, S^{n}$, or $\mathbb{M}^{n}$. Let $v$ be a vector field corresponding to some isometric motion of $\mathbb{R}^{n}$, $S^{n}$, or $\mathbb{M}^{n}$. Suppose that the points where $v$ is tangent to the double bubble separate the bubble into at least four pieces. Then the normal component of $v$ vanishes on any smooth component of the bubble which is separated into four pieces by these points of tangency. 


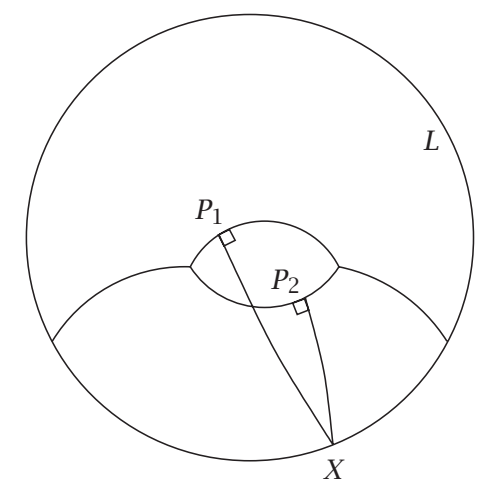

FIGURE 7.1. A bubble which can be separated by cutting at points from which orthogonal lines intersect the axis of revolution at the same point.

SKeTCH OF THE PROOF. Clearly $v$ has vanishing second variation of area. Some nontrivial linear combination of the restrictions of $v$ to the four pieces vanishes on one piece, respects the two volume constraints to first order, and has second variation zero. By stability, the normal component of $v$ must be an eigenfunction. By unique continuation for eigenfunctions, it must vanish on associated parts of the bubble which meet smoothly the piece where $v$ vanishes. Thus the normal component of $v$ will vanish on any smooth component of the bubble which is separated into four pieces by these points of tangency. See [11, Proposition 5.2] for details.

7.1. Stability in $S^{n}$. Let $\Sigma \subset S^{n}$ be a smooth double bubble of revolution about an axis $L$, with a generating curve $\Gamma$ in the upper hemisphere of $S^{2}$ that consists of arcs $\bar{\Gamma}_{i}$, with interiors $\Gamma_{i}$ ending either at the axis or in threes in vertices $v_{i j k}$. Let $\Sigma_{i}$ be the portion of $\Sigma$ generated by $\Gamma_{i}$.

We consider the map $f: \cup \Gamma_{i} \rightarrow L / \pi$ which maps each $p \in \cup \Gamma_{i}$ to the pair of antipodal points $N(p) \cap L$, where $N(p)$ denotes the geodesic line normal to $\Gamma$ at $p$.

THEOREM 7.2. Consider an area-minimizing double bubble $\Sigma \subset S^{n}, n \geq 3$, with axis of revolution $L$. Assume that there are a finite number of points $\left\{p_{1}, \ldots, p_{k}\right\}$ in $\cup \Gamma_{i}$ with $x=f\left(p_{1}\right)=\cdots=f\left(p_{k}\right)$ which separate $\Gamma$. Assume further that $\left\{p_{1}, \ldots, p_{k}\right\}$ is a minimal set with this property. Then every component $\Sigma_{i}$ which contains one of the points $p_{i}$ is part of a sphere centered at $x$.

Proof. The fact that the $p_{i}$ all map to the same point $x$ and separate $\Sigma$ means that we can separate $\Sigma$ into two pieces by cutting at points where a rotation vector field $v$ about a line $L^{\prime}$ orthogonal to $L$ through $x$ is tangent to $\Sigma$. (See Figure 7.1.) We further cut $\Sigma$ along the geodesic plane spanned by $L$ and $L^{\prime}$, again giving cuts at points where $v$ is tangent to $\Sigma$.

We now have four total pieces, and by Proposition 7.1 it follows that the normal component of $v$ vanishes on each smooth component of $\Sigma$ which is separated into four pieces by the cuts. Any of the $\Gamma_{i}$ which contains one of the $p_{i}$ corresponds to such 


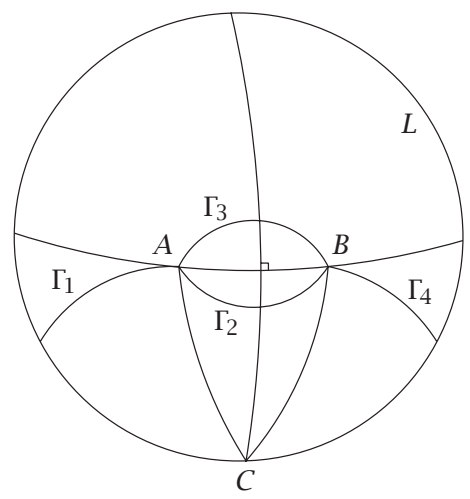

FIGURE 7.2. Cutting a possible competitor to the standard double bubble in $S^{n}$ in which all three regions are connected and two intersect the axis of rotation $L$. (Note that here $L$ is the boundary circle.)

a smooth component of $\Sigma$. Thus, since this component is both a surface of revolution about $L$ and invariant under one-parameter rotation about $L^{\prime}$, it must be spherical.

Proposition 7.3. An area-minimizing double bubble in $S^{n}$ in which all three regions are connected must be the standard double bubble.

Proof. By Corollary 6.7, there are two kinds of nonstandard competitors. One is a topological sphere intersecting the axis of revolution with a toroidal band around it, and the other is two adjacent toroidal bands that do not intersect the axis of revolution.

We first consider a competitor that intersects the axis of revolution, $L$. The generating curve $\Gamma$ is made up of four components. Two of them, $\Gamma_{1}$ and $\Gamma_{4}$ are portions of circles which intersect the axis orthogonally, and the other two, $\Gamma_{2}$ and $\Gamma_{3}$, do not. Let $\Gamma_{1}, \Gamma_{2}$, and $\Gamma_{3}$ meet at $A$, and let $\Gamma_{2}, \Gamma_{3}$, and $\Gamma_{4}$ meet at $B$. Draw a geodesic line between $A$ and $B$. Choose one of the two points where the perpendicular bisector of this line intersects $L$ and label it $C$. Note that $A$ and $B$ are equidistant from $C$. (See Figure 7.2.)

If $\Gamma_{2}$ and $\Gamma_{3}$ are not pieces of circles centered at $C$, then there are points $p_{2} \in \Gamma_{2}$ and $p_{3} \in \Gamma_{3}$ that are either closest to or farthest from $C$. (If one is a piece of such a circle, we choose any point on it.) The points $p_{2}$ and $p_{3}$ separate $\Gamma$, and the geodesic segments $\overline{p_{2} C}$ and $\overline{p_{3} C}$ both intersect $\Gamma$ orthogonally. Thus $f\left(p_{2}\right)=f\left(p_{3}\right)$, so by Theorem 7.2, $\Gamma_{2}$ and $\Gamma_{3}$ are pieces of the same circle centered at $C$, a contradiction. We conclude that a bubble of this type cannot be minimizing.

Next we consider a competitor that does not intersect $L$. (See Figure 7.3.) The generating curve $\Gamma$ is made up of three components $\Gamma_{1}, \Gamma_{2}, \Gamma_{3}$ which all meet at two points $A$ and $B$. Using the same method as above, we can find a point $p_{i}$ on each $\Gamma_{i}$ such that $f\left(p_{1}\right)=f\left(p_{2}\right)=f\left(p_{3}\right)$, and use Theorem 7.2 to derive a contradiction. We conclude that a bubble of this type cannot be minimizing. 


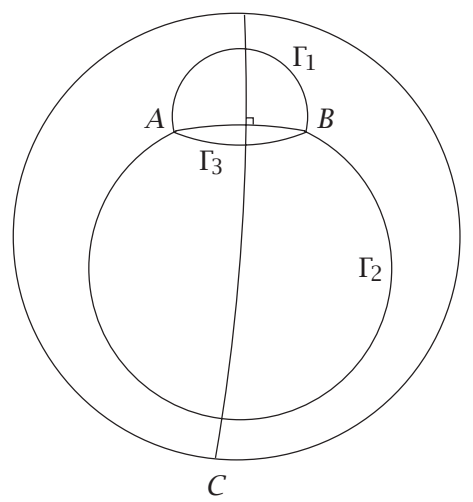

FIGURE 7.3. Cutting a possible competitor to the standard double bubble in $S^{n}$ in which all three regions are connected and only one intersects the axis of rotation $L$. (Note that here $L$ is the boundary circle.)

7.2. Stability in $\mathbb{\boxplus}^{n}$. We first need some definitions to describe lines in the hyperbolic plane, $\mathbb{M}^{2}$.

Definition 7.4. Two geodesic lines $L_{1}, L_{2} \in \mathbb{H}^{2}$ are parallel if they do not intersect, but for any positive $\epsilon$, we can find a point on each line such that the distance between the two points is less than $\epsilon$.

In the Poincaré disc model of $\mathbb{\boxplus}^{2}$, two parallel lines meet at the same point on the disc's boundary.

Definition 7.5. Two geodesic lines $L_{1}, L_{2} \in \mathbb{U}^{2}$ are disjoint if they do not intersect and there is some positive $\epsilon$ such that if we choose a point on each line, the distance between the two points is always at least $\epsilon$.

Note that disjoint lines cannot be parallel. Now that we have these definitions, we can define a function $f$ analogous to the one we defined in spherical space.

Let $\Sigma \subset \mathbb{a}^{n}$ be a smooth double bubble of revolution about an axis $L$, with a generating curve $\Gamma$ in the upper hemisphere of $S^{2}$ that consists of $\operatorname{arcs} \bar{\Gamma}_{i}$, with interiors $\Gamma_{i}$ ending either at the axis or in threes in vertices $v_{i j k}$. Let $\Sigma_{i}$ be the portion of $\Sigma$ generated by $\Gamma_{i}$.

Define $N(p)$ to be the normal line to $\Gamma$ at a point $p \in \cup \Gamma_{i}$. We consider the map $f: \cup \Gamma_{i} \rightarrow(\{0,1\} \times L) \cup\{-\infty, \infty\}$, which we define as follows. If $N(p)$ intersects $L$, then $f$ maps $p$ to $(0,(N(p) \cap L))$. If $N(p)$ is parallel to $L$, then $f$ maps $p$ to $-\infty$ or $\infty$, depending on which end of $L$ comes arbitrarily close to $N(p)$. Finally, if $N(p)$ and $L$ are disjoint, then we take the unique line $L^{\prime}$ which intersects both $L$ and $N(p)$ orthogonally, and $f$ maps $p$ to $\left(1,\left(L^{\prime} \cap L\right)\right)$.

THEOREM 7.6. Consider an area-minimizing double bubble $\Sigma \subset \mathbb{Q}^{n}, n \geq 3$, with axis of revolution L. Assume that there are a finite number of points $\left\{p_{1}, \ldots, p_{k}\right\}$ in $\cup \Gamma_{i}$ which separate $\Gamma$, with $x=f\left(p_{1}\right)=\cdots=f\left(p_{k}\right)$. Assume further that $\left\{p_{1}, \ldots, p_{k}\right\}$ is a minimal set with this property. 


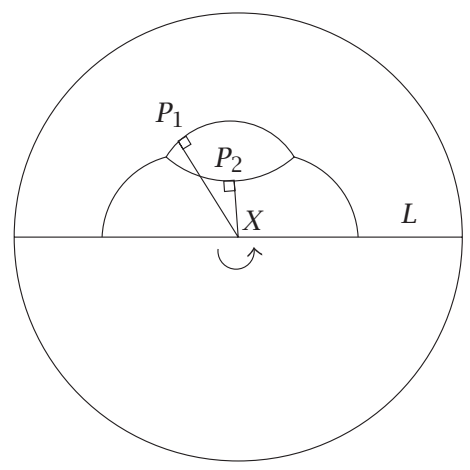

FIGURE 7.4. Generating curve for a possible nonstandard minimizer, and a set of points $p_{i}$ from which orthogonal lines meet the axis of revolution at the same (finite) point.

Then every component $\Sigma_{i}$ which contains one of the points $p_{i}$ is one of the following.

(i) Part of a sphere centered at the second coordinate of $x$, if $x$ is of the form $(0, q)$, with $q \in L$.

(ii) Part of a horosphere centered at the appropriate end of $L$, if $x$ is $\pm \infty$.

(iii) Part of a hyposphere intersecting $L$ orthogonally, if $x$ is of the form $(1, q)$, with $q \in L$. This hyposphere is generated by revolving about $L$ a hypocircle that is a constant distance from the line $L^{\prime}$ orthogonal to $L$ through $q$. (If $p_{i} \in L^{\prime}$, then this hypocircle is $L^{\prime}$, and $\Sigma_{i}$ is a geodesic plane intersecting $L$ orthogonally.)

PROof. We consider the three cases separately, as they require use of elliptic, parabolic, and hyperbolic isometries of $\mathbb{a}^{n}$, respectively, to achieve the required result. In each case, we pick an appropriate isometry, and cut $\Sigma$ into four pieces at points where the vector field $v$ of this isometry is tangent to $\Sigma$. (For a good reference on the isometries of hyperbolic space, see Beardon's book [1].)

We first consider the elliptic case, in which $x$ is of the form $(0, q)$ for some $q \in L$. We choose our isometry to be the elliptic isometry corresponding to one-parameter rotation of a circle $C$ centered at $q$ and intersecting $L$ orthogonally. The fact that the $p_{i}$ all map to the same $x$ and separate $\Gamma$ means that we can separate $\Sigma$ into two pieces by cutting at points where the vector field $v$ of this isometry is tangent to $\Sigma$. (See Figure 7.4.) We further cut $\Sigma$ along the $(n-1)$-dimensional geodesic plane containing $L$ that is orthogonal to $C$, again giving cuts at points where $v$ is tangent to $\Sigma$.

We next consider the parabolic case, in which $x$ is $\pm \infty$. We choose our isometry to be the parabolic isometry corresponding to one-parameter rotation of a horocycle $H$ centered at the appropriate end of $L$. (Note that $H$ must intersect $L$ orthogonally.) The fact that the $p_{i}$ all map to the same infinity and separate $\Gamma$ means that we can separate $\Sigma$ into two pieces by cutting at points where the vector field $v$ of this isometry is tangent to $\Sigma$. (See Figure 7.5.) We further cut $\Sigma$ along the $(n-1)$-dimensional geodesic plane containing $L$ that is orthogonal to $H$, again giving cuts at points where $v$ is tangent to $\Sigma$. 


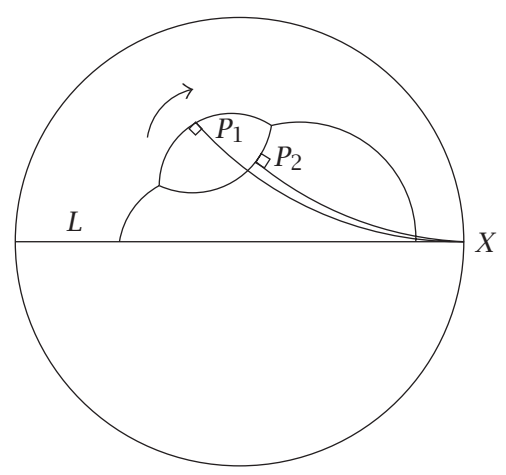

FIGURE 7.5. Generating curve for a possible nonstandard minimizer, and a set of points $p_{i}$ from which orthogonal lines meet the axis of revolution at the same infinity.

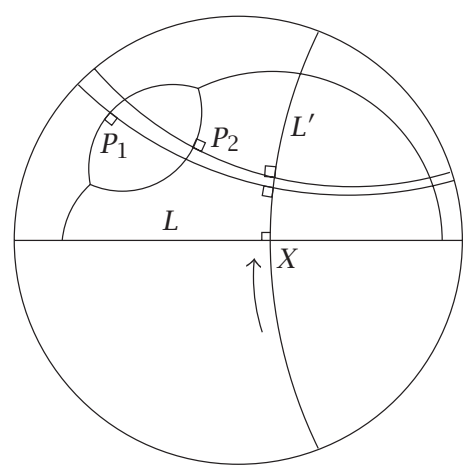

FIGURE 7.6. Generating curve for a possible nonstandard minimizer, and a set of points $p_{i}$ from which orthogonal lines intersect the same geodesic orthogonal to the axis of revolution orthogonally.

Finally, we consider the hyperbolic case, in which $x$ is of the form $(1, q)$ for some $q \in L$. We choose our isometry to be the hyperbolic isometry corresponding to oneparameter translation along a geodesic line $L^{\prime}$ orthogonal to $L$ and passing through $q$. The fact that the $p_{i}$ all map to the same point $x$ and separate $\Gamma$ means that we can separate $\Sigma$ into two pieces by cutting at points where the vector field $v$ of this isometry is tangent to $\Sigma$. (See Figure 7.6.) We further cut $\Sigma$ along the $(n-1)$-dimensional geodesic plane containing $L$ that is orthogonal to $L^{\prime}$, giving cuts at points where $v$ is tangent to $\Sigma$.

In each case, we now have four total pieces, and by Proposition 7.1 it follows that the normal component of $v$ vanishes on each $\Sigma_{i}$ which is separated into four pieces by the cuts. Each of the $\Gamma_{i}$ which contains one of the $p_{i}$ correspond to such a $\Sigma_{i}$. Thus, since this component is both a surface of revolution about $L$ and invariant under the appropriate isometry, it must be part of a sphere (if the isometry is elliptic), part of a horosphere (if the isometry is parabolic), or part of a hyposphere or goedesic plane (if the isometry is hyperbolic). 


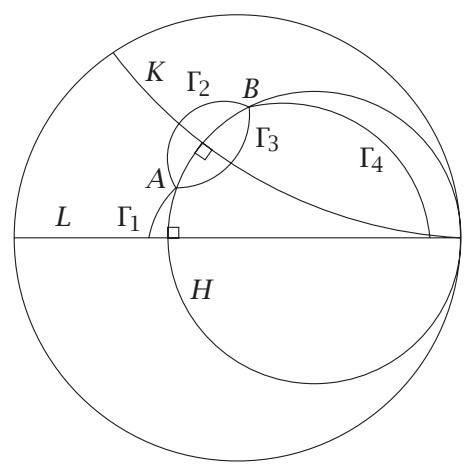

FIGURE 7.7. Using a parabolic isometry to show that a nonstandard bubble in $\mathbb{U}^{n}$ where each region is connected is unstable.

Proposition 7.7. An area-minimizing double bubble in $\mathbb{\boxplus}^{n}$ in which both regions are connected must be the standard double bubble.

Proof. By Corollary 6.12, there is only one nonstandard competitor: a topological sphere intersecting the axis of revolution, with a toroidal band around it. The generating curve $\Gamma$ of this competitor is made up of four components. Two of them, $\Gamma_{1}$ and $\Gamma_{4}$, are portions of circles, horocycles, or hypocircles which intersect the axis orthogonally, and the other two, $\Gamma_{2}$ and $\Gamma_{3}$, do not intersect the axis. Let $\Gamma_{1}, \Gamma_{2}$, and $\Gamma_{3}$ meet at $A$, and let $\Gamma_{2}, \Gamma_{3}$, and $\Gamma_{4}$ meet at $B$. Draw a geodesic segment between $A$ and $B$ and draw its perpendicular bisector $K$. Note that $A$ and $B$ are equidistant from any point on $K$.

Suppose that $K$ intersects $L$ at a point $C$. We can then use Theorem 7.6 and the same argument as in Proposition 7.3 to derive a contradiction.

Next, suppose $K$ is parallel to $L$. Then there is a unique horocycle $H$ through $A$ and $B$ that intersects both $K$ and $L$ orthogonally. If $\Gamma_{2}$ and $\Gamma_{3}$ are not pieces of horocycles a constant distance from $H$, then there are points $p_{2} \in \Gamma_{2}$ and $p_{3} \in \Gamma_{3}$ that are either closest to or farthest from $H$. (If one is a piece of such a horocycle, we choose any point on it.) The points $p_{2}$ and $p_{3}$ separate $\Gamma$, and the geodesic lines through $p_{2}$ and $p_{3}$ that intersect $\Gamma$ orthogonally also intersect $H$ orthogonally, and are thus parallel to each other and to $L$. (See Figure 7.7.) Thus $f\left(p_{2}\right)=f\left(p_{3}\right)$, so by Theorem 7.2, $\Gamma_{2}$ and $\Gamma_{3}$ are both pieces of the horocycle $H$, a contradiction.

Finally, suppose $K$ and $L$ are disjoint. Then there is a unique geodesic line $G$ that intersects both $K$ and $L$ orthogonally. If $\Gamma_{2}$ and $\Gamma_{3}$ are not pieces of hypocircles a constant distance from $G$, then there are points $p_{2} \in \Gamma_{2}$ and $p_{3} \in \Gamma_{3}$ that are either closest to or farthest from $G$. (If one is a piece of such a hypocircle, we choose any point on it.) The points $p_{2}$ and $p_{3}$ separate $\Gamma$, and the geodesic lines through $p_{2}$ and $p_{3}$ that intersect $\Gamma$ orthogonally also intersect $G$ orthogonally. (See Figure 7.8.) Thus $f\left(p_{2}\right)=f\left(p_{3}\right)$, so by Theorem $7.2, \Gamma_{2}$ and $\Gamma_{3}$ are both pieces of the same hypocircle a constant distance from $G$, the final contradiction.

7.3. Further structure of minimizers in $S^{n}$. In this section, we use the above instability argument (Theorem 7.2) to further reduce the possibilities for the structure 


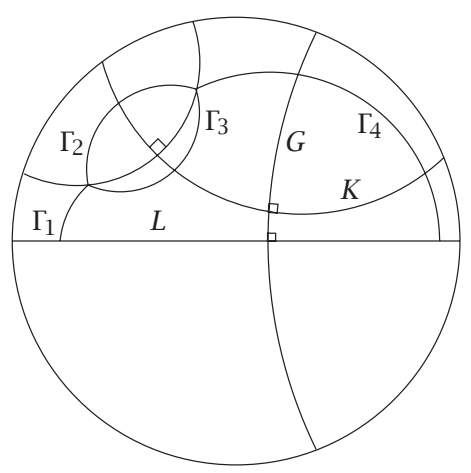

FIGURE 7.8. Using a hyperbolic isometry to show that a nonstandard bubble in $\mathbb{\sharp}^{n}$ where each region is connected is unstable.

of minimizing double bubbles in $S^{n}$. The result is a stronger structure theorem for the case in which two of the three regions are known to be connected (Theorem 7.9). This section is not necessary for our main theorems in Section 8.

LEMMA 7.8. Let $\Gamma_{i}$ be a smooth component of the generating curve of an areaminimizing double bubble in $S^{n}$. Then the set of geodesic lines orthogonal to $\Gamma_{i}$ sweeps out an angle of at most 180 degrees along the bubble's axis of symmetry L.

Proof. Suppose not. Then two such orthogonal lines must hit the axis $L$ at identical points, and thus by Theorem $7.2, \Gamma_{i}$ must be a piece of a circle centered on $L$. The set of lines orthogonal to a circular arc hitting the axis orthogonally, however, sweeps out an angle of zero along the axis, a contradiction.

THEOREM 7.9. Any nonstandard area-minimizing double bubble in $S^{n}$ in which at least two of the three regions are connected is one of the following.

(1) If the bubble intersects the axis of rotation, then it consists of a topological sphere with one toroidal band around it, and some number of toroidal bands around that. This bubble corresponds to the " $1+k$ " bubble of Reichardt et al. [17]. (See Figure 6.9a.)

(2) If the bubble does not intersect the axis of rotation, then it consists of a single toroidal band with some number of toroidal bands adjacent to it belonging to the disconnected region. Furthermore, the disconnected region must have at least three components. (See Figure 6.9b.)

Proof. We rule out item (3) in Corollary 6.6 and show that the disconnected region in item (2) must have at least three components.

Consider the generating curve of item (3). There are two smooth curves $\Gamma_{1}$ and $\Gamma_{2}$ bounding the component of the (possibly) disconnected region which contains the axis of symmetry. We claim that for at least one of $\Gamma_{1}, \Gamma_{2}$, the set of lines orthogonal to $\Gamma_{i}$ sweeps out more than 180 degrees along the axis of symmetry, contradicting Lemma 7.8. Since the $\Gamma_{i}$ are smooth, the function $f$ which determines the points at which the orthogonal lines hit the axis is continuous for each of these curves. Since the two curves meet at a 120-degree angle, $f$ is discontinuous at these points when 


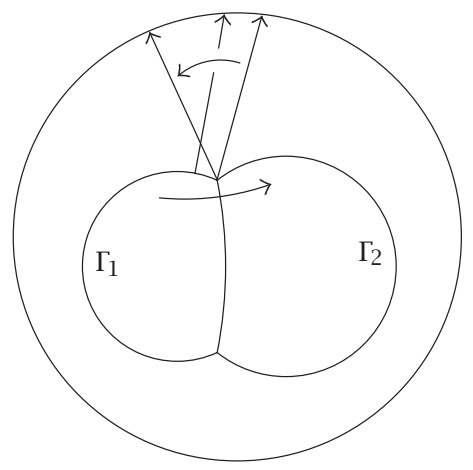

FIGURE 7.9. The function $f$ backtracks at the points of intersection of the exterior components.

considered as a function on both. The function $f$ sweeps out more than 360 degrees because at these points it backtracks when going around the closed curve $\Gamma_{1} \cup \Gamma_{2}$, due to the fact that the 120-degree angle is convex when considered from the side containing the axis (see Figure 7.9). Thus at least one of the two curves $\Gamma_{1}, \Gamma_{2}$ must sweep out more than 180 degrees, a contradiction.

The above argument rules out the case in which all three regions in item (2) are connected. We now proceed to rule out a disconnected region with two components. Consider the boundary of the region touching the axis. Let the four components of the boundary be: $\Gamma_{1}$ and $\Gamma_{2}$, forming the boundary between the region touching the axis and the disconnected region, and $\Gamma_{3}$ and $\Gamma_{4}$, forming the boundary between the region touching the axis and the other connected region (see Figure 7.10). Again, due to the fact that the 120-degree angles are convex when considered from the region containing the axis, the function $f$ sweeps out more than 360 degrees. By Lemma 7.8, $\Gamma_{1}$ must sweep out at most 180 degrees, so the rest of the curve must sweep out more. Also, the image $f\left(\Gamma_{1}\right)$ is connected. Consider the amount further swept out by $f\left(\Gamma_{3}\right)$. If $f\left(\Gamma_{3}\right) \cap f\left(\Gamma_{4}\right)$ is not empty, then we can cut both to separate the bubble, and by Theorem 7.2 both must be pieces of circles meeting the axis orthogonally. If both are such, then $f\left(\Gamma_{2}\right)$ must sweep out more than 180 degrees, for $f\left(\Gamma_{1} \cup \Gamma_{3} \cup \Gamma_{4}\right)$ would have swept out at most 180 degrees, contradicting Lemma 7.8. Thus $f\left(\Gamma_{3}\right) \cap f\left(\Gamma_{4}\right)$ is empty. But then $f\left(\Gamma_{1} \cup \Gamma_{3} \cup \Gamma_{4}\right)$ must sweep out less than 180 degrees in order for $f\left(\Gamma_{1}\right)$ not to sweep out 180 degrees and $f\left(\Gamma_{3}\right)$ and $f\left(\Gamma_{4}\right)$ to be disjoint, so $f\left(\Gamma_{2}\right)$ must sweep out more than 180 degrees, again a contradiction.

Note that this method generalizes to show that any minimizing bubble with one connected region which contains the entire axis of symmetry (item (2) in Theorem 6.5) cannot have as its graph structure a straight line.

\section{Proof of the double bubble conjecture for two equal volumes in $S^{3}$ and $\mathbb{M}^{3}$}

Proof of TheOrem 1.1 $\left(S^{3}\right)$. By Proposition 2.3, a minimizer exists. By Proposition 5.1 , both regions are connected, and the exterior is connected when its volume is at 


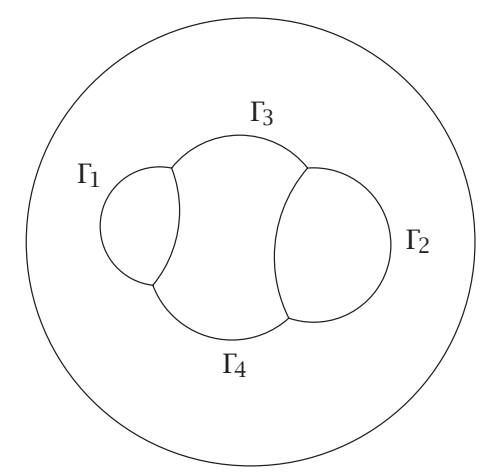

FIGURE 7.10. Generating curve for an (unstable) nonstandard double bubble in $S^{n}$ where the single disconnected region has two components.

least 10 percent of the total volume of $S^{3}$. (Hence by Corollary 6.7, a nonstandard minimizer must be either a topological sphere intersecting the axis with a toroidal band around it or two adjacent toroidal bands not intersecting the axis.) By Proposition 7.3 (the instability argument), the minimizer must be the standard double bubble (which is unique by Proposition 2.6).

Proof of Theorem 1.2 $\left(\mathbb{\square}^{3}\right)$. By Proposition 2.3, a minimizer exists. By Proposition 5.2 (and Corollary 4.5), both regions (and the exterior) are connected. (Hence by Corollary 6.12, a nonstandard minimizer must be a topological sphere intersecting the axis with a toroidal band around it.) By Proposition 7.7 (the instability argument), the minimizer must be the standard double bubble (which is unique by Proposition 2.6).

\section{Appendices}

\section{A. Program used in the proof of Proposition 5.8}

(* Area and Volume of Equal-Volume Double Bubble in $\mathrm{S}^{\wedge} 3 *$ )

(* $r<\mathrm{pi} / 2 *)$

$\mathrm{Ab} 1\left[r_{-}\right]:=$

$2 * \operatorname{Pi} *\left(1-1 / \operatorname{Sqrt}\left[1+3 / 4 *(\operatorname{Tan}[r])^{\wedge} 2\right]\right)+$

$2 * 2 * \operatorname{Pi} *(\operatorname{Sin}[r])^{\wedge} 2 *\left(1+1 /\left(2 * \operatorname{Sqrt}\left[1+3 / 4 *(\operatorname{Tan}[r])^{\wedge} 2\right]\right)\right)$

$(* \mathrm{r}>\mathrm{pi} / 2 *)$

$\mathrm{Ab} 2\left[\mathrm{r}_{-}\right]:=$

$2 * \operatorname{Pi} *\left(1+1 / \operatorname{Sqrt}\left[1+3 / 4 *(\operatorname{Tan}[r])^{\wedge} 2\right]\right)+$

$2 * 2 * \operatorname{Pi} *(\operatorname{Sin}[r])^{\wedge} 2 *\left(1-1 /\left(2 * \operatorname{Sqrt}\left[1+3 / 4 *(\operatorname{Tan}[r])^{\wedge} 2\right]\right)\right)$

(* all $r *$ )

$\mathrm{Vb}\left[r_{-}\right]:=$

$\mathrm{Pi} / 2 *(2 * r-\operatorname{Sin}[2 * r]) *\left(1+\operatorname{Cos}[r] /\left(2 * \operatorname{Sqrt}\left[1-1 / 4 *(\operatorname{Sin}[r])^{\wedge} 2\right]\right)\right)+$

$\mathrm{Pi} *(\operatorname{ArcTan}[\operatorname{Sqrt}[2] * \operatorname{Sin}[r] / \operatorname{Sqrt}[7+\operatorname{Cos}[2 * r]]]-$

Sqrt $[2] * r * \operatorname{Cos}[r] / \operatorname{Sqrt}[7+\operatorname{Cos}[2 * r]])$ 


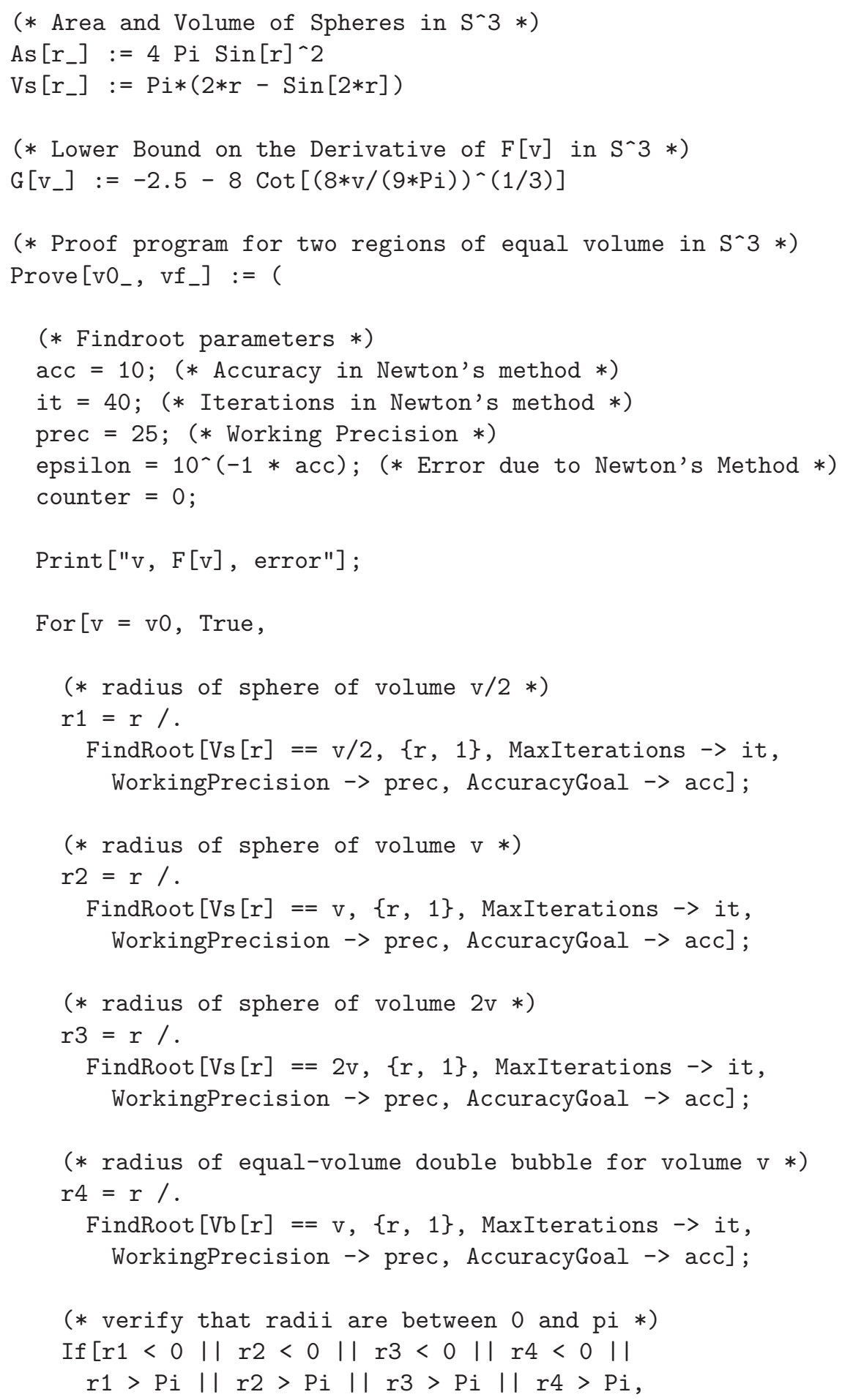




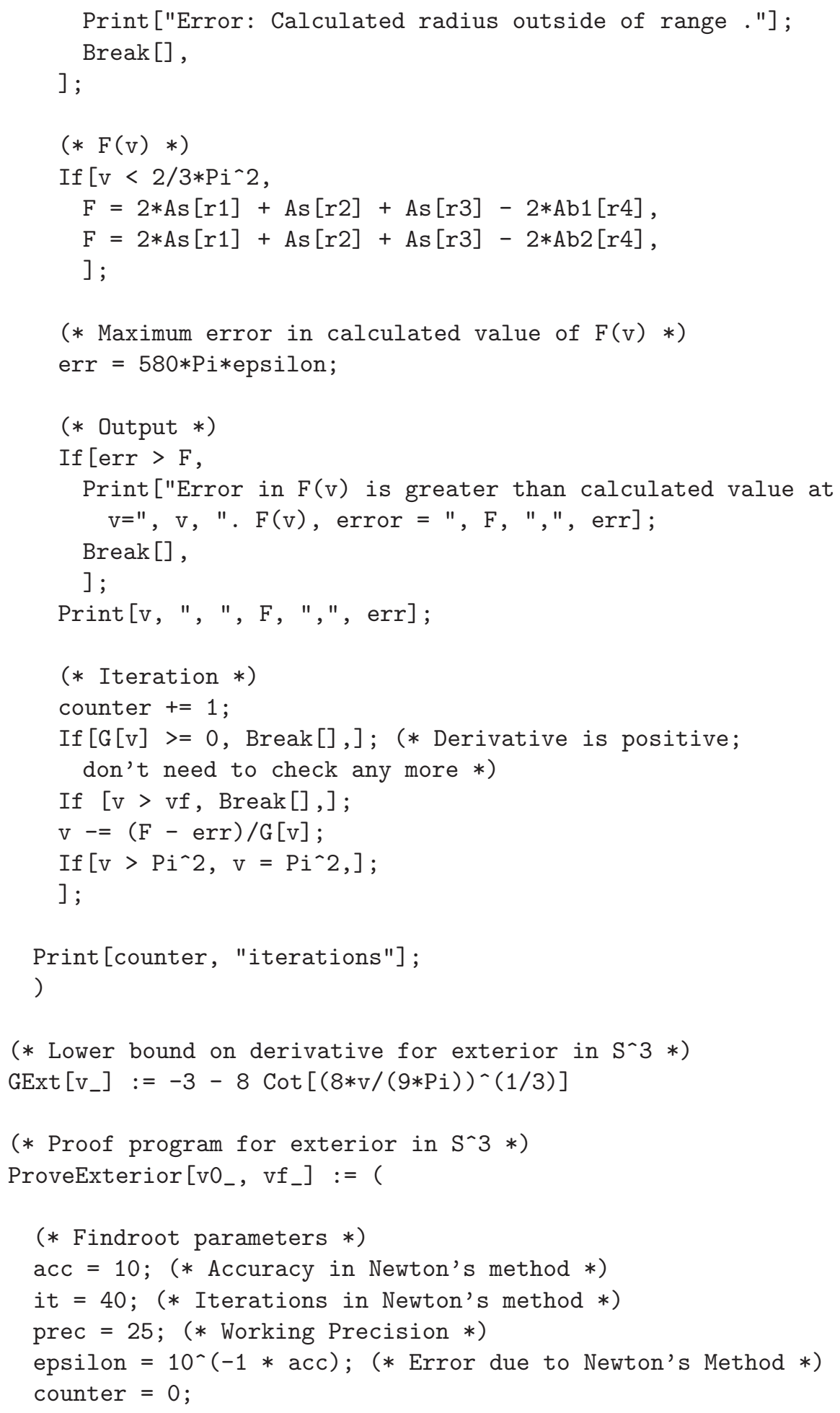




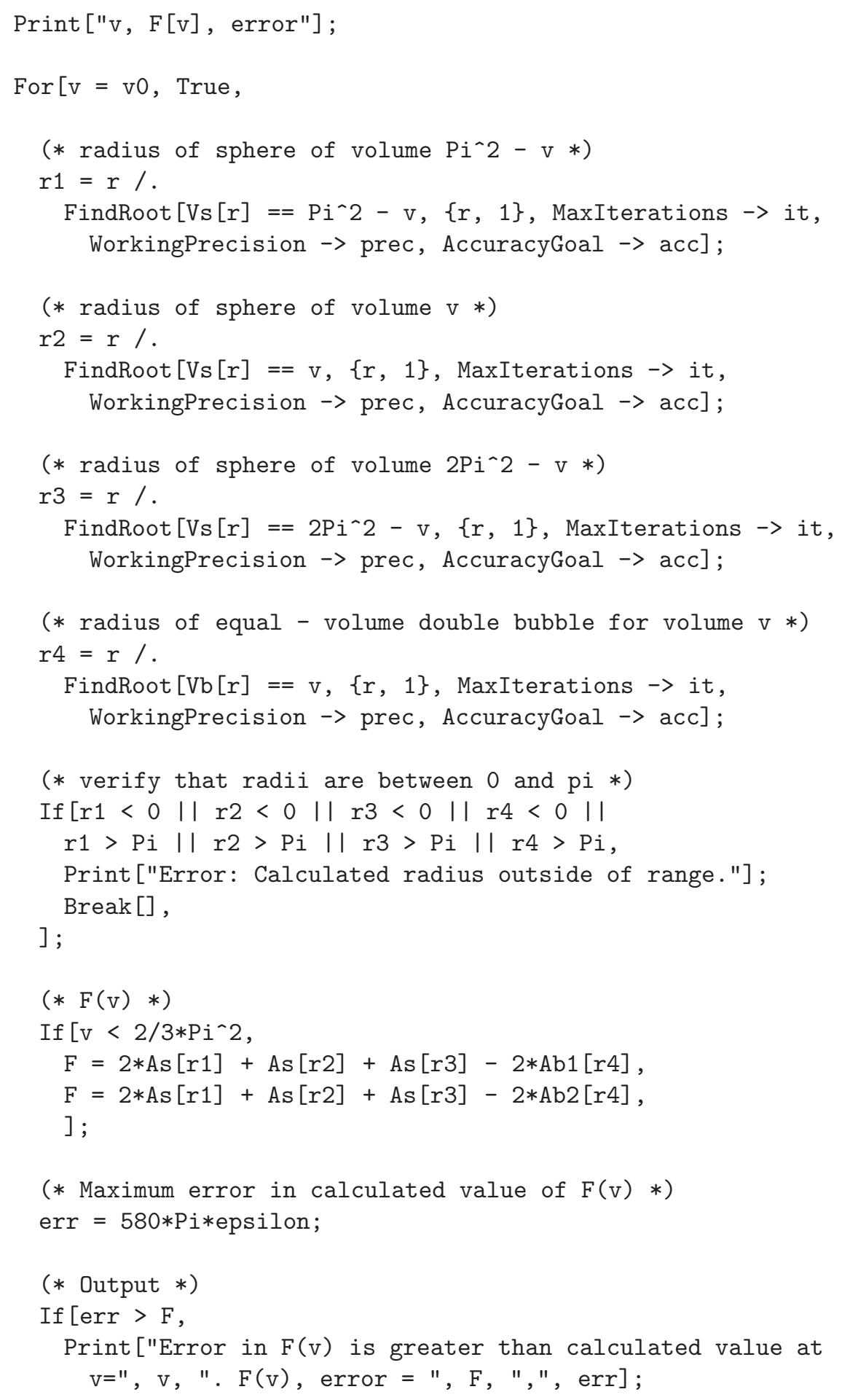




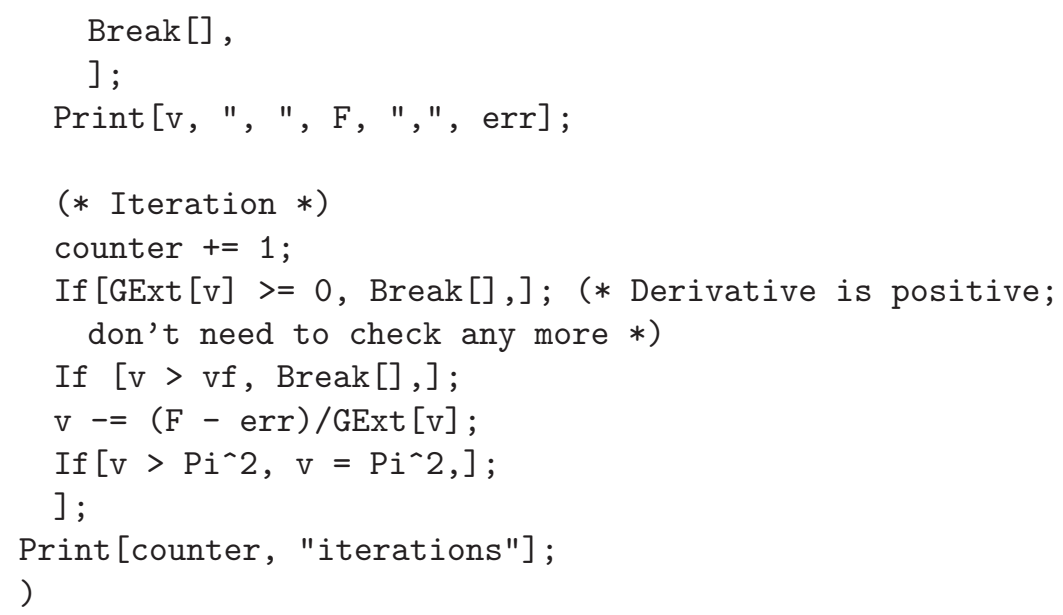

\section{B. Program used in the proof of Proposition 5.14}

(* Area and Volume of Equal - Volume Double Bubble in $\mathrm{H}^{\wedge} 3 *$ ) $\mathrm{Ab}\left[\mathrm{r}_{-}\right]:=$

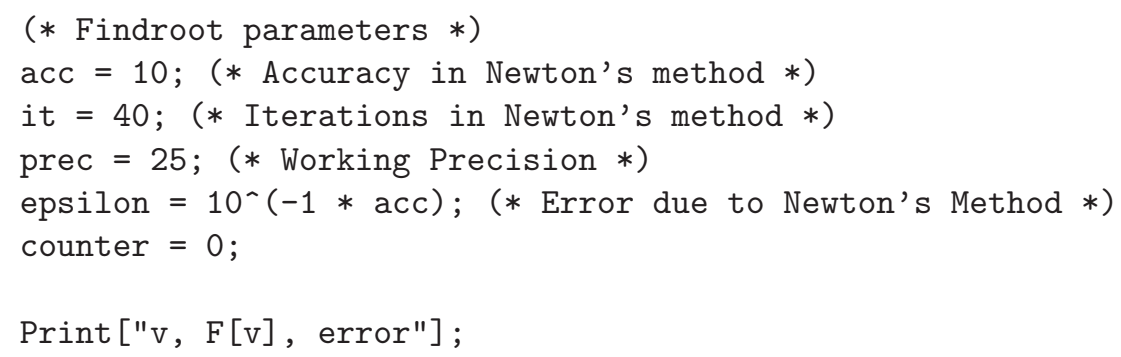




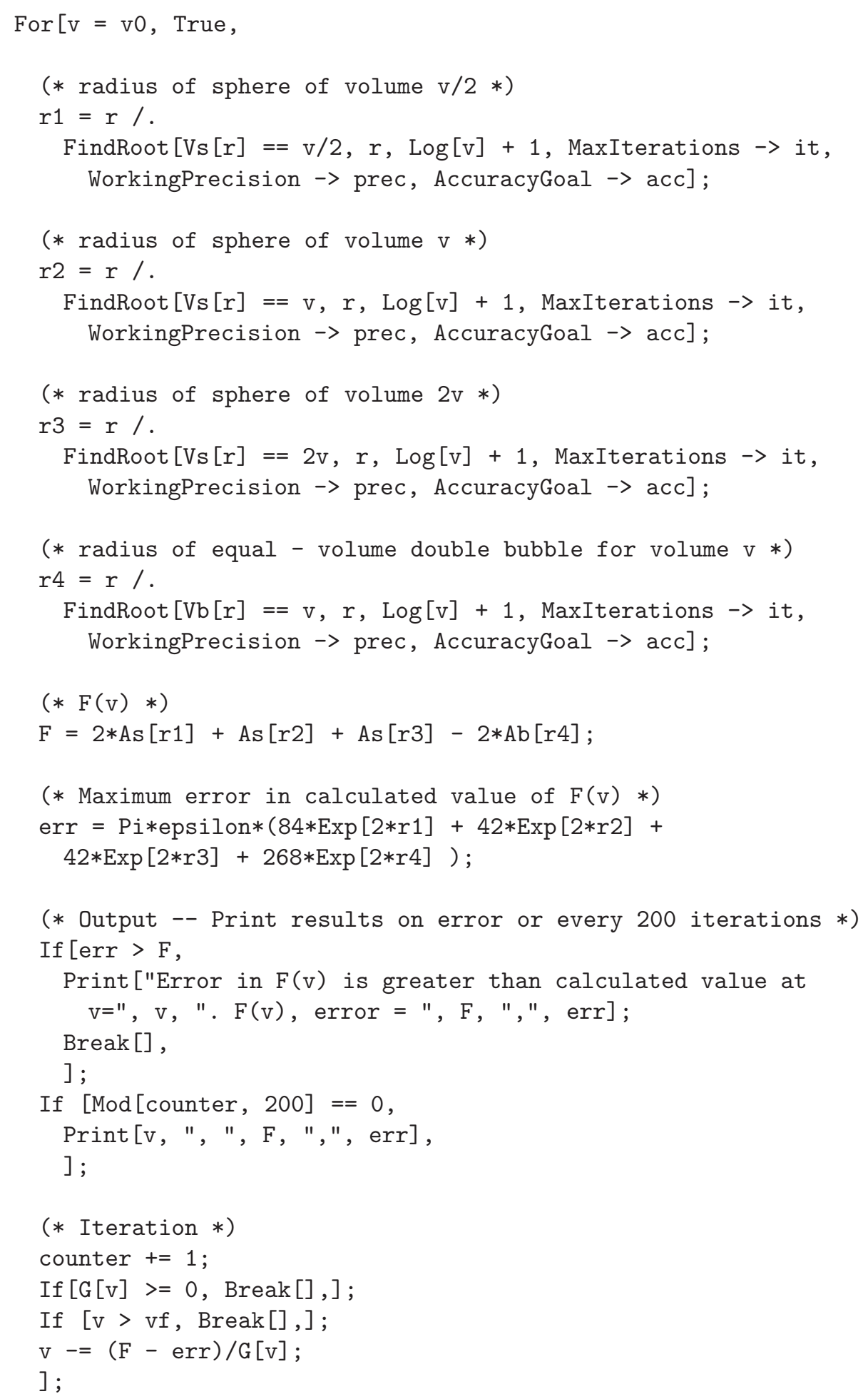




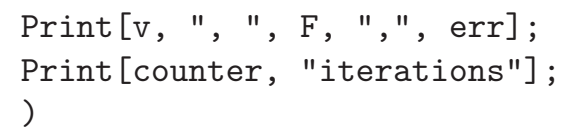

ACKNOWLEDGments. The authors were members of the 2000 Geometry Group in the Williams College NSF "SMALL" undergraduate research program. Their work was partially funded by the National Science Foundation and Williams College. They would like to thank Professor Frank Morgan for his invaluable advising. They would also like to thank their colleague John Spivack for his interest and encouragement, and members of the 2001 and 2002 "SMALL" Geometry Groups-Joseph Corneli, Paul Holt, George Lee, Nicholas Leger, and Eric Schoenfeld-for their assistance in revision.

\section{REFERENCES}

[1] A. F. Beardon, The Geometry of Discrete Groups, Graduate Texts in Mathematics, vol. 91, Springer-Verlag, New York, 1983.

[2] M. Carrión, J. Corneli, G. Walsh, and S. Beheshti, Double bubbles in the 3-torus, preprint, 2002, http://www.arXiv.org/math.DG/0208120.

[3] P. Castillon, Sur les surfaces de révolution à courbure moyenne constante dans l'espace hyperbolique [On surfaces of revolution with constant mean curvature in hyperbolic space], Ann. Fac. Sci. Toulouse Math. (6) 7 (1998), no. 3, 379-400 (French).

[4] J. Corneli, P. Holt, N. Leger, and E. Schoenfeld, Partial results on double bubbles in $S^{3}$ and $\mathbb{R}^{3}$, NSF "SMALL" undergraduate research geometry group report, Williams College, Massachusetts, 2001.

[5] J. Foisy, Soap bubble clusters in $\mathbb{R}^{2}$ and $\mathbb{R}^{3}$, undergraduate thesis, Williams College, Massachusetts, 1991.

[6] J. Foisy, M. Alfaro, J. Brock, N. Hodges, and J. Zimba, The standard double soap bubble in $\mathbb{R}^{2}$ uniquely minimizes perimeter, Pacific J. Math. 159 (1993), no. 1, 47-59.

[7] J. de M. Gomes, Spherical surfaces with constant mean curvature in hyperbolic space, Bol. Soc. Brasil. Mat. 18 (1987), no. 2, 49-73.

[8] J. Hass, M. Hutchings, and R. Schlafly, The double bubble conjecture, Electron. Res. Announc. Amer. Math. Soc. 1 (1995), no. 3, 98-102.

[9] J. Hass and R. Schlafly, Double bubbles minimize, Ann. of Math. (2) 151 (2000), no. 2, 459-515.

[10] M. Hutchings, The structure of area-minimizing double bubbles, J. Geom. Anal. 7 (1997), no. 2, 285-304.

[11] M. Hutchings, F. Morgan, M. Ritoré, and A. Ros, Proof of the double bubble conjecture, Ann. of Math. 155 (2002), no. 2, 459-489.

[12] J. D. Masters, The perimeter-minimizing enclosure of two areas in $S^{2}$, Real Anal. Exchange 22 (1996/97), no. 2, 645-654.

[13] F. Morgan, Geometric Measure Theory. A Beginner's Guide, 3rd ed., Academic Press, California, 2000.

[14] _ Proof of the double bubble conjecture, Amer. Math. Monthly 108 (2001), no. 3, 193-205.

[15] F. Morgan and W. Wichiramala, The standard double bubble is the unique stable double bubble in $\mathbb{R}^{2}$, Proc. Amer. Math. Soc. 130 (2002), no. 9, 2745-2751.

[16] J. G. Ratcliffe, Foundations of Hyperbolic Manifolds, Graduate Texts in Mathematics, vol. 149, Springer-Verlag, New York, 1994.

[17] B. W. Reichardt, C. Heilmann, Y. Y. Lai, and A. Spielman, Proof of the double bubble conjecture in $\mathbb{R}^{4}$ and certain higher dimensional cases, to appear in Pacific J. Math.

[18] I. Sterling, A generalization of a theorem of Delaunay to rotational $W$-hypersurfaces of $\sigma_{l}$-type in $\mathbb{\sharp}^{n+1}$ and $S^{n+1}$, Pacific J. Math. 127 (1987), no. 1, 187-197. 
[19] J. M. Sullivan and F. Morgan, Open problems in soap bubble geometry, Internat. J. Math. 7 (1996), no. 6, 833-842.

Andrew CotTon: Department of Mathematics, Harvard University, One OXford STREET, CAMBRIDGE, MA 02138, USA

E-mail address: acotton@fas. harvard.edu

DAVid Freeman: Department of Pure Mathematics and Mathematical Statistics, Centre for mathematical Sciences, University of CAmbridge, Wilberforce RoAd, CAMBRIDGE CB3 OWB, UK

E-mail address: dsfreeman@post. harvard.edu 


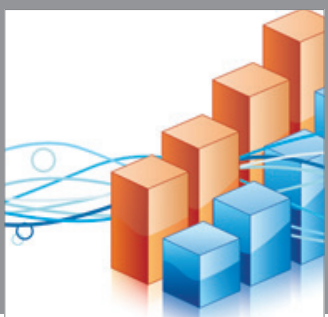

Advances in

Operations Research

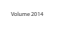

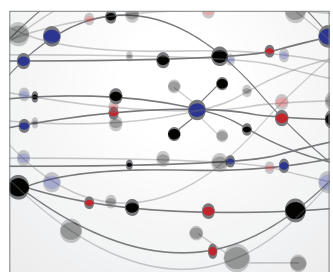

\section{The Scientific} World Journal
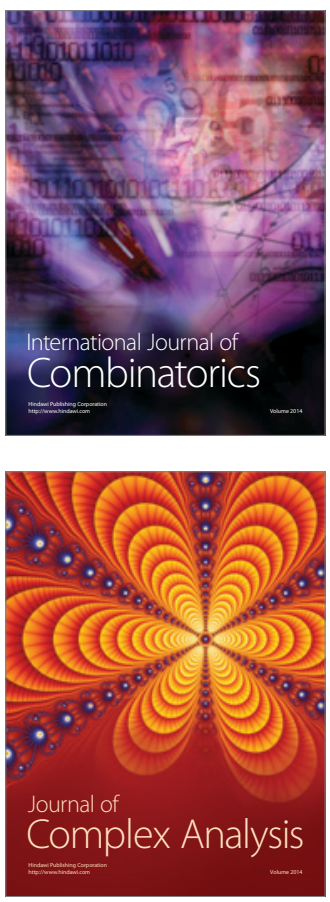

International Journal of

Mathematics and

Mathematical

Sciences
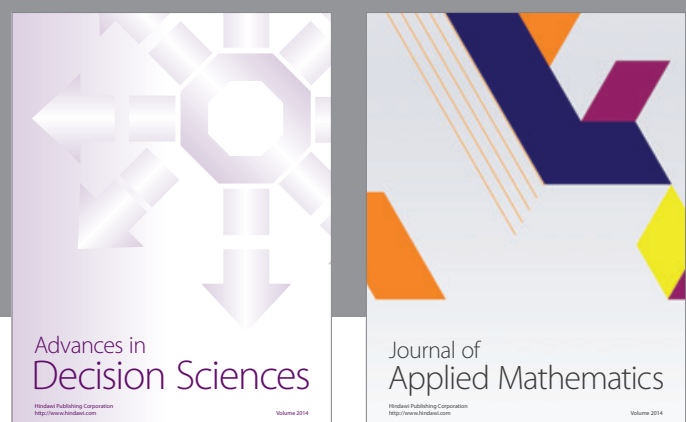

Journal of

Applied Mathematics
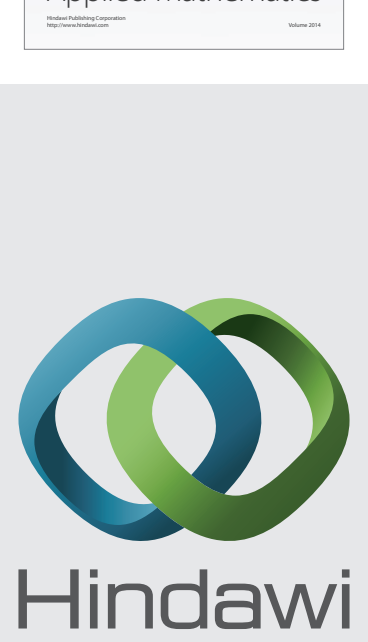

Submit your manuscripts at http://www.hindawi.com
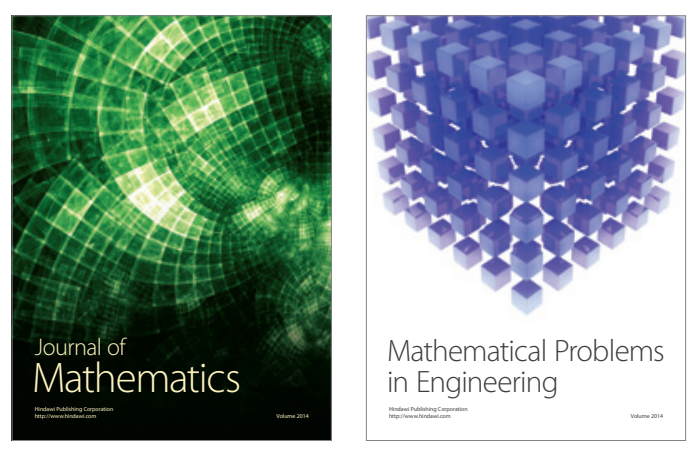

Mathematical Problems in Engineering
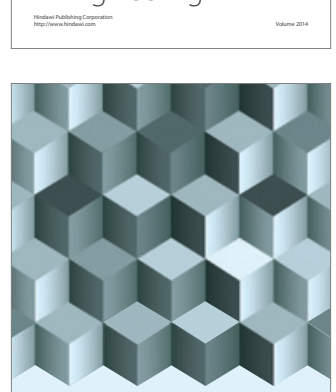

Journal of

Function Spaces
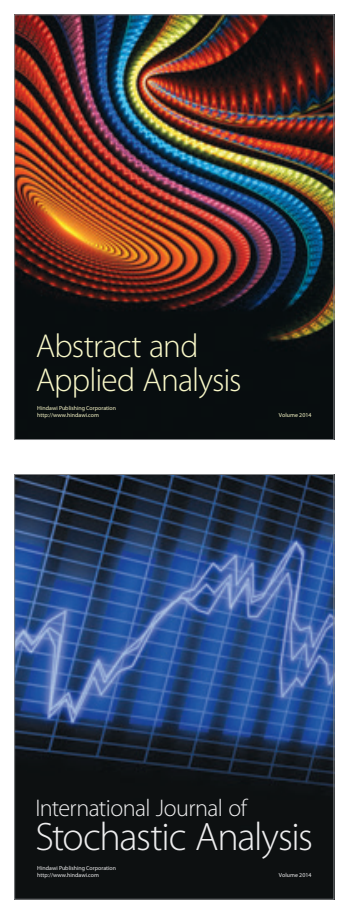

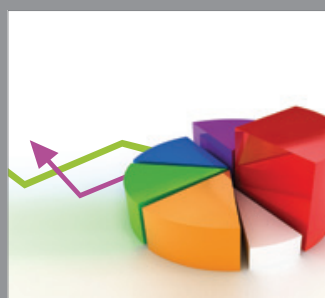

ournal of

Probability and Statistics

Promensencen
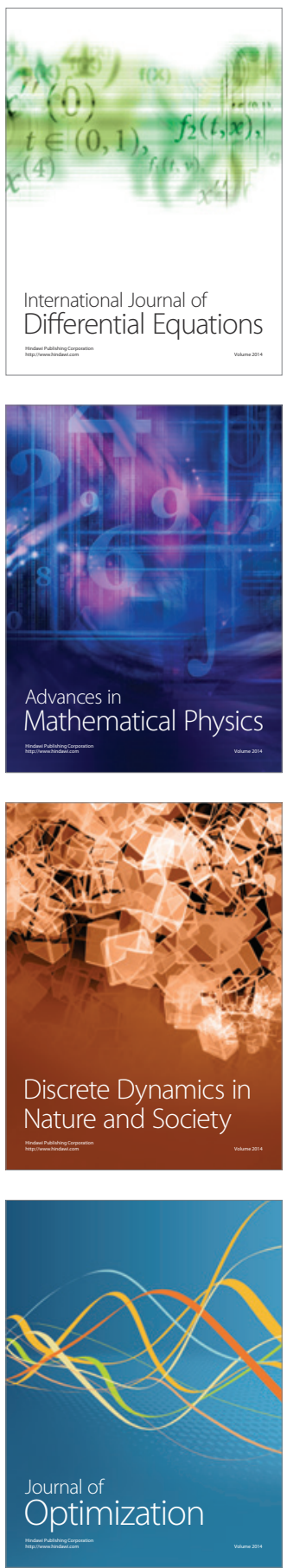